\title{
Structure and rheology of monodisperse and bimodal emulsions
}

by

Nicholas Monahan

A thesis

submitted to the Victoria University of Wellington

in partial fulfilment of the

requirements for the degree of

Master of Science with Honours

in Chemistry.

Victoria University of Wellington

2010 



\begin{abstract}
Emulsions are the basis for many commercial products such as foodstuffs and paint due in part to their highly tunable flow properties. It is qualitatively understood that factors such as the dispersed phase droplet size and size distribution should affect how an emulsion flows because they influence how droplets can deform or pack. Since standard emulsification techniques such as blending and homogenization cannot produce emulsions with well-defined size distributions, little work has been done to, in particular, quantitatively determine the influence of droplet size distribution on emulsion flow properties. Consequently, in this investigation we have probed how the droplet size distribution affects emulsion flow properties by using model monodisperse emulsion systems with narrow, controllable droplet size distributions.
\end{abstract}

Using a microfluidic flow focusing device, dodecane-in-water emulsions with diameters between 50 to $100 \mu \mathrm{m}$ with polydispersities less than $5 \%$ were produced, as characterized by pulsed field gradient nuclear magnetic resonance and optical microscopy. Due to the relatively large size of the droplets, it was only possible to examine the creamed phase of the emulsion. Samples of known polydispersity were made by mixing known quantities of two monodisperse emulsions. The monodisperse and bimodal emulsions were then subjected to rotational and oscillatory shear flow using a controlled stress rheometer to determine the effects of droplet size and size distribution on emulsion flow properties.

Rotational and oscillatory rheological experiments showed that the monodisperse emulsions had two distinct behaviours: foam-like with appreciable thixotropy and yield stresses as well as emulsion-like with no evident thixotropy. The transition between these two behaviours appears to happen at a critical droplet radius between 33 and $37 \mu \mathrm{m}$. The rheological properties of the bimodal emulsions was split into three distinct behaviours. In samples that could be considered a matrix of large droplets perturbed by smaller droplets, the flow properties were similar to those of the constituent emulsion with the larger droplets. Increasing the number fraction of smaller droplets to a 1:1 ratio creates an entirely new phase with significantly reduced elastic properties. Surprisingly, when the emulsion primarily consists of small droplets, the flow properties are most similar to that of the large droplets. Additionally, despite the microstructural differences, all emulsions showed flow characteristics typical of soft glassy materials above the glass transition temperature.

These results demonstrate the significant influence of microstructure on emulsion rheology, where altering the droplet size or polydispersity essentially creates a new phase with its own unique flow properties that is not simply a combination of the properties of the individual monodisperse components that make up the sample. 



\section{Acknowledgments}

I'm always disappointed when I read a thesis and the acknowledgments section is bland and emotionless. Perhaps it's because that person was smarter and better organised than I am, resulting in them needing very little help. Maybe it's because they're cold and heartless. Either way, I think it's important that everyone who's been there for the last two years gets a genuine, heartfelt show of appreciation. Seeing as this is a scientific work, I guess it's appropriate to mention the people who have helped me directly with my research.

My supervisor, Associate Professor Kathryn "Mavis" McGrath, is awesome. Apart from having a project that was straightforward, I really couldn't have hoped for more from working with her and the fine research students she has conned into working with her. Thanks, Kate! Sometimes I forget just how much you know about, well, almost everything. Your ability to spot an awfully convoluted idea, calm me down, put everything in perspective and motivate me is, quite frankly, awesome. Not once have I felt as if you didn't give a shit, or didn't want to see me. If I've been a pain in the arse, you've done a fine job of hiding your irritation! Despite all this, I most appreciate the fact that you've been understanding.

I also owe fellow group members Nelly Bulgarelli and Hui Lin Tan a lot of thanks for helping me get up and running with the NMR and rheology and putting up with the subsequent inane questions. Thanks also to John Ryan who honed my skills on the magnet and wasn't afraid to make a dick of himself with me by accidentally pumping several litres of water over the floor of Paul Callaghan's lab while changing a probe. Thanks to Paul's NMR group for not laughing at us too much while this happened. Petrik Galvosas helped immensely with the magnet too. In addition to Nelly and Hui Lin, Allan Raudsepp has been an absolute star with the rheometers.

I'd also like to thank Andrea Burbendorfer for helping me with the ultimately unsuccessful fabrication of my own microfluidic chips by drawing up a CAD model and guiding me through the fabrication process twice. Thanks to Epigem for donating the microfluidic devices, tubing and attachments that made this work possible, saving us several thousand dollars.

I received a whole lot of money in the form of The Curtis-Gordon Research Scholarship, the Freemasons Postgraduate Award and the J. L. Stewart Postgraduate Award. Kia ora for the money and the nice looking lines on my CV.

Now it's onto the personal stuff, I guess. Thanks to everyone else in Kate's group, past and present. Thanks to: Agathe for having an amazing accent; Ash for developing into a borderline alcoholic; Benjamin for buying us a jug at Southern Cross; Catherine for decorating Kate's book so nicely, Conrad for being older than the hills; David for wearing shoes with no socks; Graham for being foreign, Hannah for being a right-wing lunatic and sparking so much political debate; Johnny for giving me a ride to Kate's that time; Natasha for having a dinosaur t-shirt and an awesome sense of humour; $\mathrm{N}^{2}$ for being a little ray of sunshine; Riyad for his lewd sense of humour and calling Graham "Malcolm"; Sheinach for having a name that's impossibly hard to spell and William for having a goatee. You're all awesome. $\varnothing$

The other awesome people from the School of Chemical and Physical Sciences are: 
Jackie King and Gordon Heeley for doing CHEM206 with me; Dave Herman for being... unique; Alec for being a large order of magnitude nerd and starting the role playing and also for being an awesome friend; Bryan for making me look punctual and for all the car trips in the venerable Laser; John Spencer and Shaun Hendy for being an awesome lecturer and short-term supervisor respectively. They're also great letter of recommendation writers. And finally Sam for his "seven ones".

I'd also like to thank all the other nerds: Alice, Duncan, Josh and Sean. Thanks also to Andy and Chris for putting up with us on Thursday nights.

Thanks to all the munters from Napier: Andrew, Clint, Nick, Pete and Ricky for reminding me that there's life outside of chemistry and that it involves awesome music, cricket and home brew.

As for Mum, Walker and Jaime, there's little here I can say to express how grateful I am for all of your help, particularly during the time I was writing this damned thing. In an attempt to avoid writing even more, I'll keep it short: you're awesome. I love you all so much. 
1 Introduction $\quad 1$

1.1 Emulsions and their uses . . . . . . . . . . . . . 1

1.2 Research proposal ...................... 3

1.3 Thesis structure $\ldots \ldots \ldots \ldots \ldots \ldots \ldots \ldots \ldots \ldots \ldots$

2 Microfluidic methods $\quad \mathbf{5}$

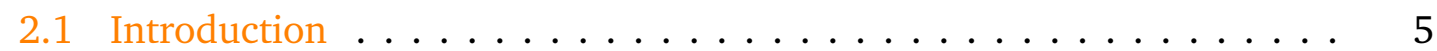

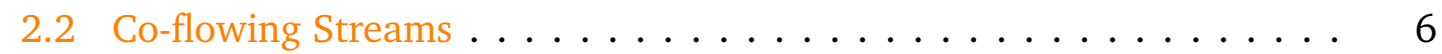

2.3 Cross flowing Streams $\ldots \ldots \ldots \ldots \ldots \ldots \ldots$

2.4 Flow focusing devices $\ldots \ldots \ldots \ldots \ldots \ldots$

2.4.1 Sub-micrometre droplets with microfluidic devices . . . . . . 9 9

2.5 Alternative routes to monodisperse emulsions . . . . . . . . . . . . 10

2.5 .1 Fractionation ................... 10

2.5.2 Shearing of Coarse Emulsions . . . . . . . . . . . . . 11

3 Nuclear magnetic resonance $\quad 13$

3.1 Molecular self-diffusion . . . . . . . . . . . . . . . . . . . 13

3.2 Diffusion and magnetic field gradients . . . . . . . . . . . . 15

3.2.1 Magnetic field gradients as spatial labels . . . . . . . . . . 15

3.2.2 Measuring diffusion with magnetic field gradients . . . . . . 16

3.2.3 Correlating signal attenuation with diffusion . . . . . . . . . 19

3.3 Emulsion characterisation . . . . . . . . . . . . . . 21

3.3.1 Polydisperse emulsions . . . . . . . . . . . . . . 21

3.3.2 Monodisperse emulsions .................. 22

3.3 .3 Non-NMR methods . . . . . . . . . . . . . . . 22

4 An introduction to rheology $\quad \mathbf{2 5}$

4.1 Hookean solids and Newtonian fluids . . . . . . . . . . . . . . 25

4.1 .1 Hookean solids . . . . . . . . . . . . . . . . . . 25

4.1 .2 Newtonian fluids . . . . . . . . . . . . . . . . . 26

4.2 Increased complexity . . . . . . . . . . . . . . . 27

4.2.1 Stress and strain in multiple dimensions . . . . . . . . . 27 
4.2 .2 Non-Newtonian fluids . . . . . . . . . . . . . . . 28

4.2 .3 Viscoelasticity ... . . . . . . . . . . . . . . 29

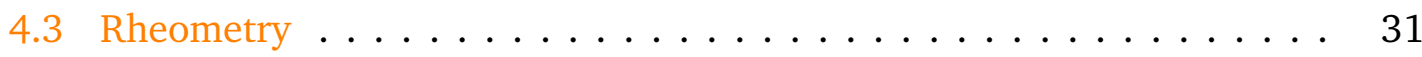

4.3 .1 Rheometers ........................ 31

4.3 .2 Measuring geometries . . . . . . . . . . . . . 32

4.3.3 Standard flows and material functions . . . . . . . . . . 35

4.4 Emulsion rheology . . . . . . . . . . . . . . . 37

4.4.1 Droplet dynamics . . . . . . . . . . . . . 37

4.4 .2 Dilute emulsions . . . . . . . . . . . . . 38

4.4 .3 Concentrated emulsions $\ldots \ldots \ldots \ldots \ldots$

4.4.4 Monodisperse emulsions . . . . . . . . . . . . . . . . . 39

5 Emulsion formation and structure $\quad 41$

5.1 Emulsion formation . . . . . . . . . . . . . . . . 41

5.1 .1 Experimental details . . . . . . . . . . . . . . 41

5.1 .2 Results and discussion $\ldots \ldots \ldots \ldots \ldots$. . . . . . 42

5.2 Emulsion characterisation . . . . . . . . . . . . 46

5.2 .1 Optical microscopy $\ldots \ldots \ldots \ldots \ldots$............. 46

5.2 .2 Nuclear magnetic resonance . . . . . . . . . . . . . . 47

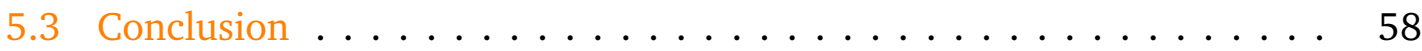

6 Emulsion rheology $\quad 61$

6.1 Steady shear ............................ 61

6.1 .1 Experimental details . . . . . . . . . . . . . 61

6.1 .2 The effect of droplet size . . . . . . . . . . . . . 62

6.1 .3 The effect of polydispersity . . . . . . . . . . . . . 64

6.2 Oscillatory shear . . . . . . . . . . . . . . . 66

6.2 .1 Experimental details . . . . . . . . . . . . . . . 66

6.2 .2 The effect of droplet size . . . . . . . . . . . . . 67

6.2 .3 The effect of polydispersity . . . . . . . . . . 70

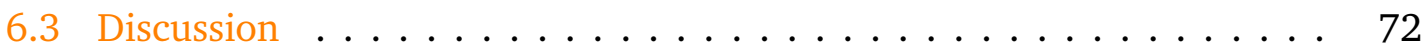

6.3 .1 General considerations . . . . . . . . . . . . . . . 72

6.3.2 Monodisperse emulsions . . . . . . . . . . . . . . 75

6.3 .3 Bimodal emulsions . . . . . . . . . . . . . . . 78

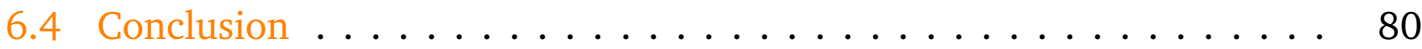


7 Conclusions and prospects $\quad \mathbf{8 1}$

7.1 Conclusions............................ 81

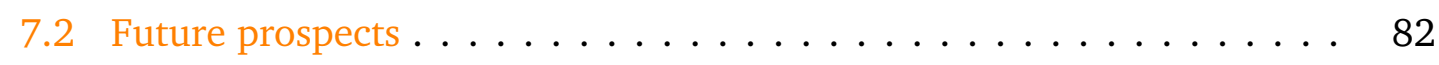

$\begin{array}{lc}\text { References } & \mathbf{8 3}\end{array}$ 



\section{List of symbols}

$\delta \quad$ Gradient pulse duration, see equation (3.14), page 16, s

$\delta \quad$ Viscoelastic phase angle, see equation (4.29), page 36, Degrees

$\dot{\gamma} \quad$ Shear rate, see equation (2.2), page $5, \mathrm{~s}^{-1}$

$\dot{\gamma} \quad$ Shear rate, see equation (4.4), page $26, \mathrm{~s}^{-}$

$\eta \quad$ Dynamic viscosity, see equation (2.1), page 5, Pa s

$\omega \quad$ Larmor frequency, see equation (3.10), page 16, $\operatorname{rad~s}^{-1}$

$\psi \quad$ Flow rate ratio, see equation (2.3), page 8

$\rho \quad$ Fluid density, see equation (2.1), page $5, \mathrm{~g} \mathrm{~cm}^{3}$

$\sigma \quad$ Shear stress, see equation (4.1), page 25, $\mathrm{Pa}$

g Magnetic field gradient, see equation (3.11), page 16, $\mathrm{T} \mathrm{m}^{-1}$

q Scattering vector, see equation (3.23), page 20, $\mathrm{m}^{-1}$

$\xi \quad$ Interfacial tension, see equation (2.2), page $5, \mathrm{mN} \mathrm{m}^{-1}$

a Droplet radius, see equation (3.27), page $21, \mathrm{~m}$

$D \quad$ Self-diffusion coefficient, see equation (3.3), page $13, \mathrm{~m}^{2} \mathrm{~s}^{-1}$

E Signal attenuation, see equation (3.18), page 18

$G^{\prime \prime} \quad$ Viscoelastic loss modulus, see equation (4.32), page 36, Pa

$G^{\prime} \quad$ Viscoelastic storage modulus, see equation (4.31), page 36, $\mathrm{Pa}$

Q Flow rate, see equation (2.3), page $8, \mathrm{Lh}^{-1}$ 



\section{Introduction}

\subsection{Emulsions and their uses}

Emulsions are a colloidal dispersion where one liquid is dispersed through a continuous phase of a second, immiscible liquid often in the presence of a surfactant [1]. In most emulsions one phase is aqueous, while the second is a hydrophobic oil. Consequently, there are two general classifications for emulsions: oil-in-water emulsions $(\mathrm{O} / \mathrm{W})$, where an oil is dispersed through an aqueous continuous phase; and water-in-oil $(\mathrm{W} / \mathrm{O})$ emulsions, where the aqueous phase is dispersed through an oil. A typical emulsion is depicted schematically in Figure 1.1. Emulsions occur naturally and synthetically, with a number of examples listed in Table 1.1.

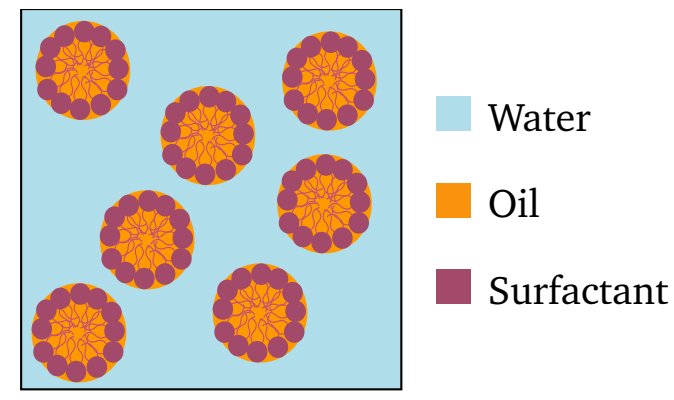

Figure 1.1: A schematic representation of an oil-in-water emulsion, not to scale. To lower the interfacial energy, the hydrophobic "head" of the surfactant sits at the oil-water interface while the hydrophobic tails penetrate the oil droplet. This situation is reversed for water-in-oil emulsions.

Most emulsions do not form spontaneously, and a large amount of energy must be supplied to the bulk phases to induce emulsification [2]. This is a result of the emulsified form having a much larger interfacial area than the two separated bulk phases. For example, when forming an $\mathrm{O} / \mathrm{W}$ emulsion every subdivision step where mean droplet radius is halved increases the interfacial area by a factor of 2 , with an appropriate increase in total surface energy required [3]. While an emulsion will eventually revert to its thermodynamically stable form [4], the addition of a surfactant provides kinetic non-lability by adsorbing at the droplet interface. As a result, some emulsions may last thousands of years [5]. The addition of a surfactant also reduces the energy required for emulsification by lowering the interfacial tension [6].

The wide range of uses and applications of emulsions is a result of their highly variable physical properties. Such properties are controlled by the chemical composition of the continuous and dispersed phases, the mean droplet size $\bar{x}$, droplet volume fraction 
$\phi$, defined as the volume of the dispersed phase divided by the volume of both the dispersed and continuous phases, and the droplet polydispersity or droplet size distribution $\delta \sigma$, defined as the standard deviation of the droplet size divided by the mean droplet size [7]. For example, by altering the volume fraction, an emulsion can change from a simple viscous liquid at low $\phi$ to an elastic solid with a large shear modulus at high $\phi[8]$. Because these properties influence the feeling and ease of application of products such as cosmetic creams and lotions, they are an important aspect of consumer approval. As a result, understanding the link between microstructure and physical properties is important not only for satisfying academic curiosity, but for the development and optimisation of consumer level products [3].

The effect of microstructure alteration on physical properties is well understood qualitatively [7]. It has been shown that the viscosity of an emulsion increases as the (average) droplet size decreases or as the interfacial tension increases simply because the droplets are more resistant to deformation [9]. We also know that polydispersity should have a significant effect on emulsion flow properties because it influences how the droplets are able to pack, but we do not know how. Indeed, we do not know what effect, if any, polydispersity has had on existing studies that have defined our understanding of how emulsion rheology should be influenced by microstructure [10].

A deeper understanding is limited by the lack of model systems - it is nearly impossible to vary, for example, volume fraction while controlling all other physical variables due to the stochastic processes that occur when traditional methods of emulsification are used [11]. Emulsions are typically formed by applying a large shearing force to the bulk immiscible liquids in macroscopic mixers [2], which often unpredictably produce emulsions with droplet sizes that can vary by several orders of magnitude and a size distribution that is different at each synthesis.

The obvious solution is to create and study a model system which can be controlled in a more predictable manner [12]. Recently developed techniques have enabled the production of emulsions with low $(<0.25)$ polydispersity [8]. Such emulsions are called monodisperse. These emulsions allow for an increased understanding of the relationship between the structure of the emulsion and its properties due to the inherent control over mean droplet size, polydispersity and volume fraction.

Table 1.1: Common occurrences of emulsions, sorted by field (from [3]).

\begin{tabular}{ll}
\hline Field & Example \\
\hline Agriculture & Insecticides and herbicides \\
Foods & $\begin{array}{l}\text { Milk, butter, mayonnaise, cream liqueurs, ice cream, } \\
\text { creams, soft drink syrups, mayonnaise, sauces, margarine, } \\
\text { spreads, salad dressings, cheeses }\end{array}$ \\
Manufacturing & $\begin{array}{l}\text { Polishes, asphalt (paving) emulsion } \\
\text { Medicine }\end{array}$ \\
& $\begin{array}{l}\text { Soluble vitamin and hormone products, biological } \\
\text { membranes, blood }\end{array}$ \\
Personal care products & Hair and skin creams and lotions \\
\hline
\end{tabular}




\subsection{Research proposal}

Motivated by the lack of understanding of how polydispersity influences emulsion rheology, the main objective of this research programme was to create a series of model emulsions that could be used to establish the influence of droplet size and size distribution on the flow properties of an emulsion.

To achieve this goal, it was proposed that monodisperse emulsions be generated using a microfluidic device because of the relative experimental ease compared to previous methods devised to produce emulsions with narrow size distributions. These monodisperse emulsions were then to be used to create bimodal samples as a first approximation to true polydispersity.

Nuclear magnetic resonance (NMR) was suggested as an appropriate characterisation tool as it not only allows the droplet size and size distribution to be established, but can also give information about the microstructure of the emulsions. In particular, it was thought that diffusion of the continuous phase would identify the effect of varying degrees of polydispersity on the regularity of droplet packing.

Finally, a controlled stress rheometer was to be used to perform rotational and oscillatory rheological experiments on the monodisperse and bimodal emulsions. The rotational experiments would probe the bulk, non-linear flow properties, while the oscillatory experiments could probe the response at a short length scale, local environment. Interpretation of these rheological tests would enable us to establish whether polydispersity manifests itself as a perturbative effect or something more complicated.

\subsection{Thesis structure}

The remainder of the introductory part of this thesis spans from chapters 2 to 4 . In chapter 2 , microfluidic methods for generating monodisperse emulsions are introduced and compared to other methods for obtaining model systems. This is followed by the theoretical basis for measuring the diffusion of molecules using NMR and how this can yield microstructural information in chapter 5 . Chapter 4 is the final introductory chapter, in which is introduced the fundamental concepts in rheology and rheometry for measuring the flow properties of emulsions.

The experimental part of the thesis begins with chapter 5 , which covers the formation and subsequent characterisation of monodisperse and bimodal emulsions using a flow focusing microfluidic device and NMR, respectively. Chapter 6 then presents the results of the rheological investigation of the emulsions, including how droplet size and size distribution affect the flow properties of monodisperse and bimodal emulsions. Finally, a conclusion of the work is presented in chapter 7 as well as a section discussing the implications of the current research on future projects. 



\section{2}

Microfluidic methods

It it not possible to obtain monodisperse emulsions using conventional emulsion production techniques and special experimental approaches must be adopted to control the droplet size distribution. Such approaches include the microfluidic and other methods introduced in this chapter.

\subsection{Introduction}

The behaviour of fluids that are constrained to a sub-millimetre scale is the domain of microfluidics. By using microfabrication techniques such as lithography, it is possible to create complex channel geometries in polymeric and glass substrates with characteristic lengths as small as several micrometres [13]. In such confined environments, fluids behave differently to what is observed macroscopically [14]. The small length scales lead to low Reynolds number (Re) flows, where the fluid streams are laminar and easily controlled. The Reynolds number is a dimensionless quantity and can be defined as [15]

$$
\operatorname{Re} \equiv \frac{\rho v d}{\eta}
$$

where $\rho$ is the density, $v$ the velocity, $d$ the hydraulic diameter and $\eta$ the dynamic viscosity of the fluid. It is essentially a ratio of inertial $(\rho v)$ to viscous $(\eta)$ forces [16]. Another dimensionless number important for characterising microfluidic systems is the capillary number Ca. It can be defined in terms of the continuous phase flow field that deforms the dispersed phase to produce droplets:

$$
\mathrm{Ca} \equiv \frac{\eta_{c} G \dot{\gamma}}{\zeta}
$$

where $G$ is the capillary radius, $\dot{\gamma}$ is the shear rate imposed by the flow of the continuous phase and $\xi$ is the interfacial tension. As a ratio of viscous forces and interfacial tension, it describes how easily the interface can be deformed. The shear stress from the continuous phase serves to disrupt the structure of the dispersed phase, and the surface tension acts to restore the structure. When $\mathrm{Ca}$ is high, the structure is unstable and tends to disintegrate. When it is low, there will be little deformation.

Due to their length scales, the majority of microfluidic systems produce emulsions with droplets ranging from several to hundreds of micrometres [17]. Microfluidic devices offer considerably more control over the droplet size (within a limit dictated by the dimensions of the system) and size distribution when compared to conventional methods, with polydispersities generally between 1 and 3\% [18]. 
When forming an emulsion using a microfluidic device, both the dispersed and continuous phase fluids are introduced into separate microchannels by pressure-driven flow where the volume flow rate is controlled. Upon meeting at a junction, the dispersed phase is forced into a "jet" by the motion of the continuous phase, and due to free-surface instability, a droplet eventually pinches off from the end of the jet. Steady flow of both phases results in a continuous stream of equally-sized droplets forming a monodisperse emulsion. Droplet breakup is characterised by the competition between stresses applied by the continuous phase fluid that deform the jet, and viscosity that serves to resist deformation [19].
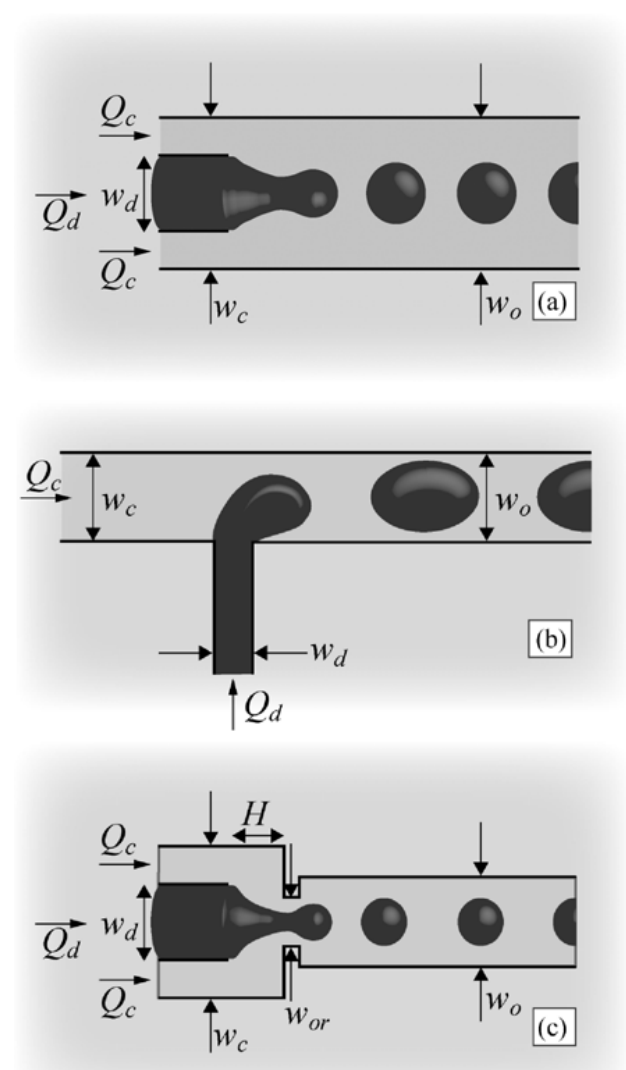

Figure 2.1: Depictions of the three main types of microfluidic geometries used to produce monodisperse emulsions. (a) Co-flowing streams, (b) cross-flowing streams, and (c) flow-focusing. $Q_{c}$ and $Q_{d}$ represent the flow rates of the continuous and dispersed phases, respectively. The widths of the inlet channels are indicated by $w_{c}$ for the continuous and $w_{d}$ for the dispersed phases. The width of the outlet channels and orifice are indicated by $w_{o}$ and $w_{o r}$, respectively. The devices are assumed to be planar and of a uniform depth $h$. Reproduced from [17].

There are three main microfluidic geometries used to generate monodisperse emulsions [20]: co-flowing streams, cross-flowing streams and flow-focusing devices. These are depicted schematically in Figure 2.1 and further discussed below. For further discussion of microfluidic device fabrication and the physics that explains the phenomena observed in these systems, see [21] and [16], respectively.

\subsection{Co-flowing Streams}

The droplet breakup in two co-flowing streams (Figure 2.1a) involves the flow of a surfactant-containing continuous phase past the end of a capillary through which the 
dispersed phase is extruded [22]. Droplets form at the tip of the capillary and detach when they reach a size where the drag due to the co-flowing continuous phase exceeds the interfacial tension. Such a geometry has been implemented as a micropipette inserted into a rotating bath containing the continuous phase [22] and as a capillary placed within a rectangular flow cell [23].

There are two distinct droplet breakup modes that operate when a capillary tip is immersed in a continuous co-flowing liquid: dripping, where droplets pinch off near the capillary tip, and jetting, where droplets pinch off from an extended thread downstream of the capillary tip [23], which are compared in Figure 2.2(a) and (b). The transition between these modes occurs when the continuous phase velocity increases above a critical value. This critical velocity decreases as the dispersed phase flow rate increases, as a result of the larger axial momentum of the forming droplet which facilitates jet formation [24]. The critical velocity also decreases as the dispersed phase viscosity increases, due to the increased resistance to deformation of the liquid-liquid interface. Finally, the critical velocity decreases with a decrease in interfacial tension force, as it is the only conservative force which holds the drop at the capillary tip, so an equilibrium of forces is reached earlier for systems with lower interfacial tension [23].

With both forms of the geometry, the droplet size can be controlled by altering experimental parameters [17]. Generally, droplets are smaller when the continuous phase velocity is faster as a larger amount of shear stress is exerted on the interface. The higher the shear stress is, the more rapidly it deforms the continuous phase stream meaning a smaller amount of the dispersed phase is able to enter the droplet. Droplet size increases with increasing dispersed phase flow rate, as a larger amount of the dispersed phase is able to enter the forming droplet prior to pinchoff. These trends are general for both breakup modes. Reducing the interfacial tension results in larger droplets due to decreased resistance to the shear stress that results in breakup. However, variations in the viscosity ratio have little effect on droplet size over a wide range of continuous phase velocities [23].

The droplet sizes produced by breakup in co-flowing streams range from $80 \mu \mathrm{m}$ up to a few hundred micrometres in diameter, with very small polydispersity values of $1 \%$ to $2 \%[22,23]$.

\subsection{Cross flowing Streams}

Cross-flowing dispersed and continuous phase streams, depicted schematically in Figure 2.1b, are typically implemented using microchannels arranged in a T-junction.

The droplet breakup process can be described as follows [26]. The two immiscible fluids form an interface at the junction of the dispersed and continuous phase channels. The stream of the dispersed phase moves into the main channel and a droplet forms. The pressure gradient and the flow of the continuous phase distort the droplet in the downstream direction. The interface on the upstream side of the droplet moves downstream. When the interface approaches the downstream edge of the dispersed phase channel, the neck connecting the droplet with the bulk of the dispersed phase breaks. The disconnected droplet flows downstream with the continuous phase, while the tip of the stream of the discontinuous phase retracts to the end of the inlet and the process repeats.

T-junction geometries can be further classified into two groups: those in which the 


\section{Co-flowing streams}

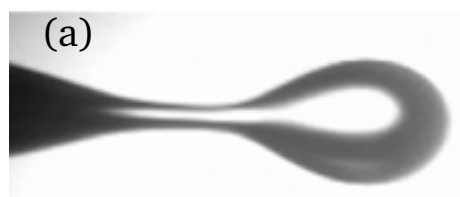

Cross-flowing streams

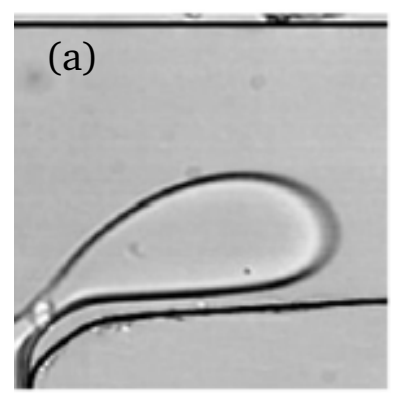

Flow focusing

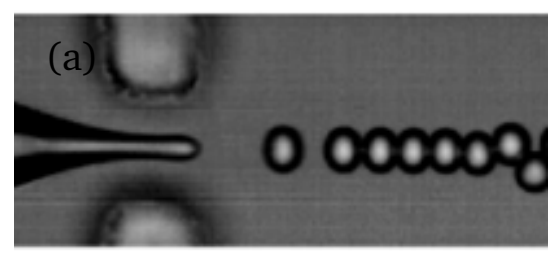

(b)
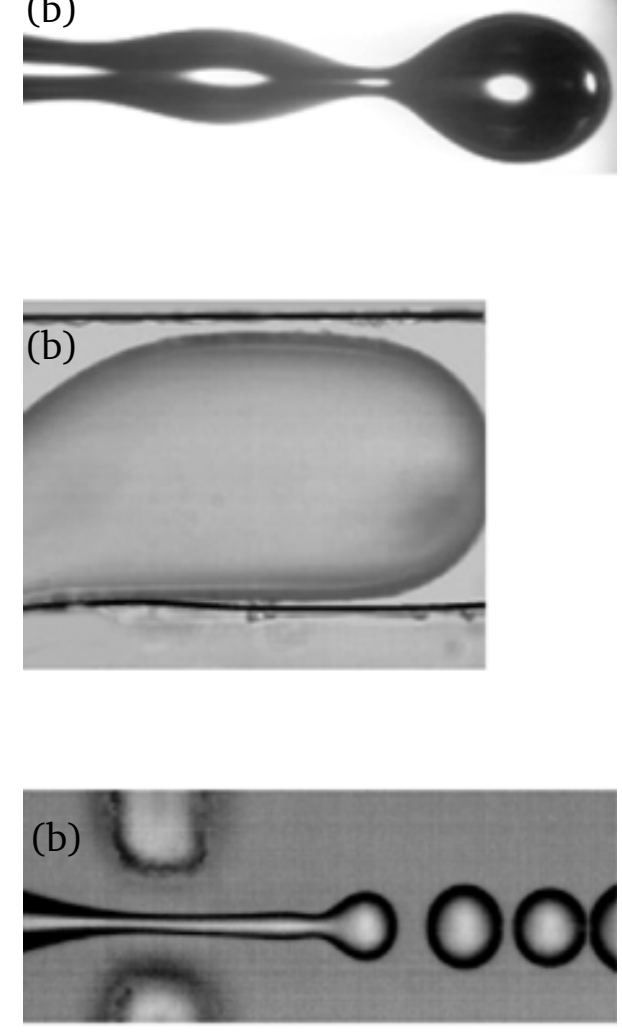

Figure 2.2: A comparison of droplet breakup in each of the three main microfluidic geometries used for droplet formation, reproduced from [17]. Dripping (a) and jetting modes (b) in co-flowing streams [23]. Unconfined (c) and confined (d) break up in cross-flowing streams [25]. Elongational flows (e) and dripping and jetting modes (f) in a flow focusing device [25].

emerging dispersed phase droplets do not interact with, or remain unconfined by, the downstream cross-channel wall and those in which the emerging droplet rapidly fills the junction and is influenced by its confinement. These classes are known as unconfined and confined breakup, respectively, and are compared in Figure 2.2c and $\mathrm{d}$ [17]. Unconfined breakup occurs when the continuous phase channel is much wider than the dispersed phase channel, while confined breakup occurs when both the continuous and dispersed phase channels have a comparable width.

Despite the geometric difference, both types of cross-flowing streams exhibit similar behaviour changes as system variables are altered. Generally, the size of the droplets is primarily controlled by the flow rate of the continuous phase, with the droplet diameter decreasing as the continuous phase flow rate increases [27]. There is, however, a critical flow rate ratio $\psi$, where droplets will no longer form. This occurs when the continuous phase flow rate is much larger than the dispersed phase flow rate. The corresponding difference in pressure means the dispersed phase liquid can never enter the junction for droplet formation to occur [28]. The flow rate ratio is defined as

$$
\psi=\frac{Q_{d}}{Q_{c}}
$$

where $Q_{d}$ and $Q_{c}$ are the flow rates of the dispersed and continuous phases, respectively. The flow rate of the dispersed phase has little to no effect on droplet size. The viscosity 
of both phases has a significant effect on droplet size [29]. High continuous phase viscosities impart a larger shear stress on the forming droplet, leading to smaller droplets as the interface is distorted more quickly. High dispersed phase viscosities increase the droplet size, as the forming droplet is more able to resist deformation [29].

\subsection{Flow focusing devices}

Flow-focusing devices involve the two phases flowing coaxially into separate channels in a planar geometry (Figure 2.1c), with the continuous phase flowing either side of the dispersed phase [17]. The two phases then meet at the tip of the centre channel and flow through a contraction, or orifice, just beyond this point. The resulting elongationdominated velocity field in the outer liquid forces the dispersed phase into a thin jet which breaks into droplets [30].

There are several breakup modes that occur in flow-focusing devices: geometry controlled, dripping, jetting and in some cases tipstreaming [25]. Dripping and jetting are compared in Figure 2.2e and f. Transitions between these modes are controlled by the capillary number and the flow rate ratio. Within each flow mode the droplet size varies with the flow parameters [17].

At low $\mathrm{Ca}$, droplets form by a geometry controlled mechanism. In the presence of small shear forces the dispersed phase finger grows a large spherical end beyond the orifice which breaks off to minimise the interfacial area. Part of the breakup mechanism produces small satellite droplets that increase the polydispersity of the resultant emulsion. By increasing the capillary number the spherical growth and therefore the droplet size is reduced. Within this mode, the minimum droplet size is highly influenced by the dimension of the orifice.

Increase of the capillary number results in droplet forming by a dripping mechanism. This is characterised by droplets forming within one characteristic diameter of the flow focusing orifice, while a portion of the dispersed phase remains left behind at a fixed point in the orifice [31-33]. The droplets generated in this mode are smaller than the size of the orifice and have a polydispersity of less than $2 \%$. As the capillary number is increased, the droplet diameter decreases and likewise with decreasing flow rate ratio. Droplet breakup in this mode is thought to be a result of capillary instabilities with end pinching combined with the viscous drag imposed on the forming droplet by the flow of the continuous phase which thins and stretches the necked region behind the droplet.

As the capillary number increases, a transition from dripping to jetting occurs. The jetting mode involves the formation of a long dispersed phase finger that extends at least three orifice diameters beyond the orifice exit [32]. The resulting droplets are larger and more polydisperse than those formed by the dripping mode. The transition is thought to occur when the timescale for visco-capillary pinchoff is comparable to the timescale for the growth of the dispersed phase jet [32].

\subsubsection{Sub-micrometre droplets with microfluidic devices}

The largest issue associated with microfluidic methods is that the minimum droplet size obtainable is large compared to conventional emulsification techniques and other methods for producing monodisperse emulsions (see sections 2.5.1 and 2.5.2). This is 
a consequence of the droplet breakup modes having some degree of dependence on the size of the microfluidic device such as the junction or orifice [34].

Large droplets are not ideal due to the effect they have on emulsion stability [35]. There is typically a density difference between the continuous and dispersed phases, which results in the emulsion droplets either rising to the surface (creaming) when they have a lower density than the continuous phase, or sinking to the bottom (sedimenting) when they have a higher density than the continuous phase. Assuming the droplets behave as solid spheres, the creaming or sedimentation velocity is given by the Stokes equation

$$
U=\frac{2 a^{2}|\Delta \rho| g}{9 \eta},
$$

where $U$ is the velocity, $a$ is the droplet radius, $\Delta \rho$ is the density difference between the dispersed and continuous phases, $g$ is the acceleration due to gravity, and $\eta$ the continuous phase viscosity. The velocity scales with $a^{2}$, resulting in large velocities for droplets the size typically produced by microfluidic methods. Consequently, the droplets will rise or sink before any meaningful analysis can be performed. It is therefore imperative that the droplet size is minimised.

Under certain conditions, it is possible to obtain sub-micrometre droplets in flowfocusing devices [31]. Within a specific range of both the capillary number and the flow rate ratio - typically $0.5 \leq \mathrm{Ca} \leq 1$ and $\psi>40-$ and a concentration of surfactant close to the critical micelle concentration, droplets are formed in the tipstreaming mode of breakup. This involves the emerging dispersed phase jet forming a conical shape with a thin thread from which the droplets break off, and is similar to processes observed with larger scale isolated droplets in unbounded linear flows [36]. While such flows have been observed, little analysis has been performed [17]. It remains, however, a potential method to expand the range of droplet sizes obtainable using a microfluidic device.

\subsection{Alternative routes to monodisperse emulsions}

Techniques such as crude emulsion fractionation and the shearing of coarse emulsions allow monodisperse emulsions to be formed that have much lower droplet sizes than emulsions generated using microfluidic methods. When several cubic centimetres of sample is required for rheological analysis the following techniques are both time consuming as well as labour intensive.

\subsubsection{Fractionation}

This technique exploits an attractive depletion interaction between droplets. Such an interaction is induced by the presence of excess micelles in the continuous phase [37]. As emulsion droplets approach each other, the inter-droplet region is depleted of micelles. Consequently a micelle concentration gradient develops and hence an osmotic pressure. The micelles diffuse out from between the droplets to reduce the concentration gradient and the droplets aggregate [38]. If the continuous and dispersed phases have an appreciable density difference, the aggregate will cream (or sediment) and can easily be removed from the bulk.

The concentration of micelles required to induce flocculation increases as the droplet size increases [39]. A clear corollary is that by gradually increasing the concentration 
of surfactant in the continuous phase, it is possible to separate fractions of droplets of the same size from a polydisperse emulsion.

It is suitable for both $\mathrm{O} / \mathrm{W}$ and $\mathrm{W} / \mathrm{O}$ emulsions, as it only requires that the surfactant of choice forms micelles in the continuous phase. It is not necessary to use the same surfacant used to originally emulsify the dispersed phase. It is limited to droplets with diameters between 0.1 and $2 \mu \mathrm{m}$ and produces polydispersities of 0.1 to 0.2 [8]. However, the method is time-consuming, and is inefficient as a large amount of the dispersed phase is not used [22].

\subsubsection{Shearing of Coarse Emulsions}

It is possible to generate monodisperse emulsions by applying a shear (tangential) force to viscoelastic emulsions with large, polydisperse droplets [40]. A crude polydisperse emulsion is first prepared by progressively incorporating oil into the continuous phase containing a surfactant. In a second step, a high shear rate is applied to the crude emulsion which becomes monodisperse after no more than a few seconds (Figure 2.3). The shear has the effect to reduce both the average droplet diameter and the distribution width [41].

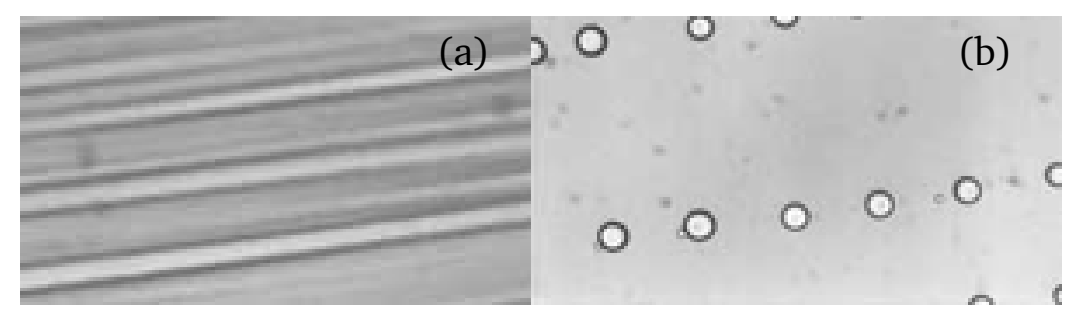

Figure 2.3: Images of monodisperse emulsion formation by shearing coarse emulsions. (a) The droplets of the precursor emulsion elongate into long cylinders under an applied shear stress, and (b) break up into aligned droplets of similar size. From [41].

The shear stress induces a Rayleigh instability in the droplets by stretching them into long cylinders. Under the driving force of surface tension, the cylinders break up into droplets to minimise their surface area. The final drop size is primarily determined by the amplitude of the applied shear stress and is slightly affected by the ratio of the dispersed and continuous phase viscosities. This latter parameter influences the distribution width and appears to be relevant to control the final monodispersity. If the viscosity ratio $p$ between the dispersed and continuous phases lies between 0.01 and 2 , the shear force applied to the coarse polydisperse emulsion leads to a monodisperse emulsion with a mean diameter directly governed by the stress [41]. The viscosity ratio is defined as

$$
p=\frac{\eta_{d}}{\eta_{c}}
$$

It is necessary that the precursor emulsion (either $\mathrm{O} / \mathrm{W}$ or $\mathrm{W} / \mathrm{O}$ ) is viscoelastic and the shear rate is well controlled. By tuning these variables, it is possible to produce emulsions with mean droplet diameters between 0.3 and $10 \mu \mathrm{m}$ with polydipersities of as little as 0.06, although typical values are closer to 0.16 [42]. Additional polydispersity may be introduced if the precursor emulsion contains droplets smaller than the mean droplet diameter of the final monodisperse emulsion [43]. 



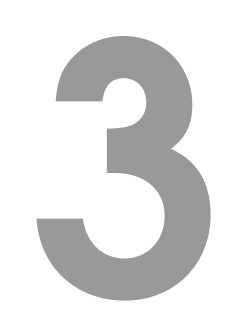

\section{Nuclear magnetic resonance}

The physical phenomenon of NMR is the result of the interaction between the magnetic moments of a nuclei and an applied magnetic field. While the spectroscopic technique based on this phenomenon is known to most chemists as an indispensable technique for molecular structural elucidation, it can also be used to probe molecular motion.

In heterogeneous systems such as emulsions, interfacial boundaries place restrictions on how molecules can diffuse through the sample. This restricted diffusion leaves a characteristic signature on the NMR signal, which can be used to obtain, for example, the droplet size distribution of an emulsion. The purpose of this chapter is to explain how diffusion is measured with NMR and what microstructural information this can provide. A more general discussion of NMR and its origins can be found in many excellent textbooks, for example [44] and [45].

\subsection{Molecular self-diffusion}

The stochastic motion of molecules in a liquid at thermal equilibrium is termed selfdiffusion. In the context of an NMR experiment, molecular self-diffusion is often modelled by a self correlation function $P_{s}\left(\mathbf{r} \mid \mathbf{r}^{\prime}, t\right)$, which describes the probability of a molecule at $\mathbf{r}$ moving to $\mathbf{r}^{\prime}$ during a time $t$ [46]. The self-correlation function can be used to determine the total probability $\Psi\left(\mathbf{r}^{\prime}, t\right)$, of finding a molecule at $\mathbf{r}^{\prime}$, at time $t$

$$
\Psi\left(\mathbf{r}^{\prime}, t\right)=\int \rho(\mathbf{r}, 0) P_{s}\left(\mathbf{r} \mid \mathbf{r}^{\prime}, t\right) d \mathbf{r}
$$

where $\rho(\mathbf{r}, 0)$ is the molecular density. Integrating over $\mathbf{r}$ accounts for all starting positions. It is useful to think of $\boldsymbol{\Psi}\left(\mathbf{r}^{\prime}, t\right)$ as an ensemble-averaged probability concentration for a single molecule because then it is possible to assume is obeys the Fick's diffusion equation with the following condition [47]

$$
P\left(\mathbf{r} \mid \mathbf{r}^{\prime}, 0\right)=\delta\left(\mathbf{r}^{\prime}-\mathbf{r}\right),
$$

where $\delta$ is the Dirac delta function. Fick's first law of diffusion can then be written as

$$
\mathbf{J}=-D \nabla P_{s},
$$

where $\mathbf{J}$ is the conditional probability flux and $D$ is the molecular self-diffusion coefficient. Because the conditional probability is conserved, we can use the continuity 
theorem to write

$$
\boldsymbol{\nabla} \cdot \mathbf{J}=-\frac{\partial P_{s}}{\partial t}
$$

Combining equations 3.3 and 3.4 gives Fick's second law of diffusion

$$
\frac{\partial P_{s}}{\partial t}=D \nabla^{2} P_{s}
$$

With the appropriate boundary conditions, equation 3.5 can be solved to give the form of the self-correlation propagator. For example, for free diffusion in a homogeneous, boundless medium, $P_{s}$ is

$$
P_{s}\left(\mathbf{r} \mid \mathbf{r}^{\prime}, t\right)=(4 \pi D t)^{-3 / 2} \exp \left(\frac{\left(\mathbf{r}^{\prime}-\mathbf{r}\right)^{2}}{4 D t}\right) .
$$

We note that the self-correlation propagator is concerned only with the net displacement, not the initial position of the molecule. Therefore, $\mathbf{r}-\mathbf{r}^{\prime}$ can be refered to as the dynamic displacement $\mathbf{R}$ (figure 3.1), and the self-correlation propagator becomes $\overline{P_{s}}(\mathbf{R}, t)$.

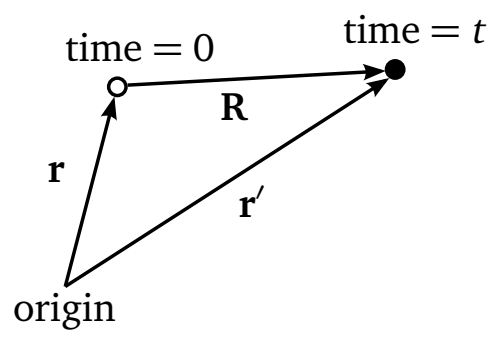

Figure 3.1: The displacement of a molecule from $\mathbf{r}$ to $\mathbf{r}^{\prime}$ over a time period $t$.

The mean squared displacement can be calculated using [48]

$$
\left\langle\mathbf{R}^{2}\right\rangle=\int_{-\infty}^{\infty} \bar{P}_{s}(\mathbf{R}, t) \mathbf{R}^{2} d \mathbf{R} .
$$

If we use $P_{s}$ for free diffusion (equation 3.6), then the mean square displacement for free diffusion is [49]

$$
\left\langle R^{2}\right\rangle=n D t,
$$

where $n=2$, 4, or 6 for one, two or three dimensions, respectively. The displacement due to diffusion increases linearly with time, and in a heterogeneous system such as an emulsion a molecule that has diffused for a sufficiently long time will come in contact with the interface. This is termed restricted diffusion.

To establish whether a molecule in such systems is experiencing free or restricted diffusion, a dimensionless variable

$$
\xi=\frac{D \Delta}{a^{2}}
$$

is used that relates the diffusion time $\Delta$, diffusion coefficient and the characteristic 
dimension of the restricting geometry $a$.

When $\xi<1$, the molecules have not diffused far enough to feel the effects of the boundary, and the diffusion coefficient is the same as that observed for the freely diffusing species. As the diffusion time increases so that $\xi \approx 1$, the observed diffusion coefficient will be smaller than that of the freely diffusing species as a portion of the molecules feel the effects of the boundary and the mean square displacement no longer varies linearly with time. At very long diffusion times, $\xi>1$ and the maximum distance a molecule can travel is limited by the boundaries, therefore the mean squared displacement and measured diffusion coefficient become independent of $\Delta$. These three modes of diffusion are shown for a sphere in figure 3.2

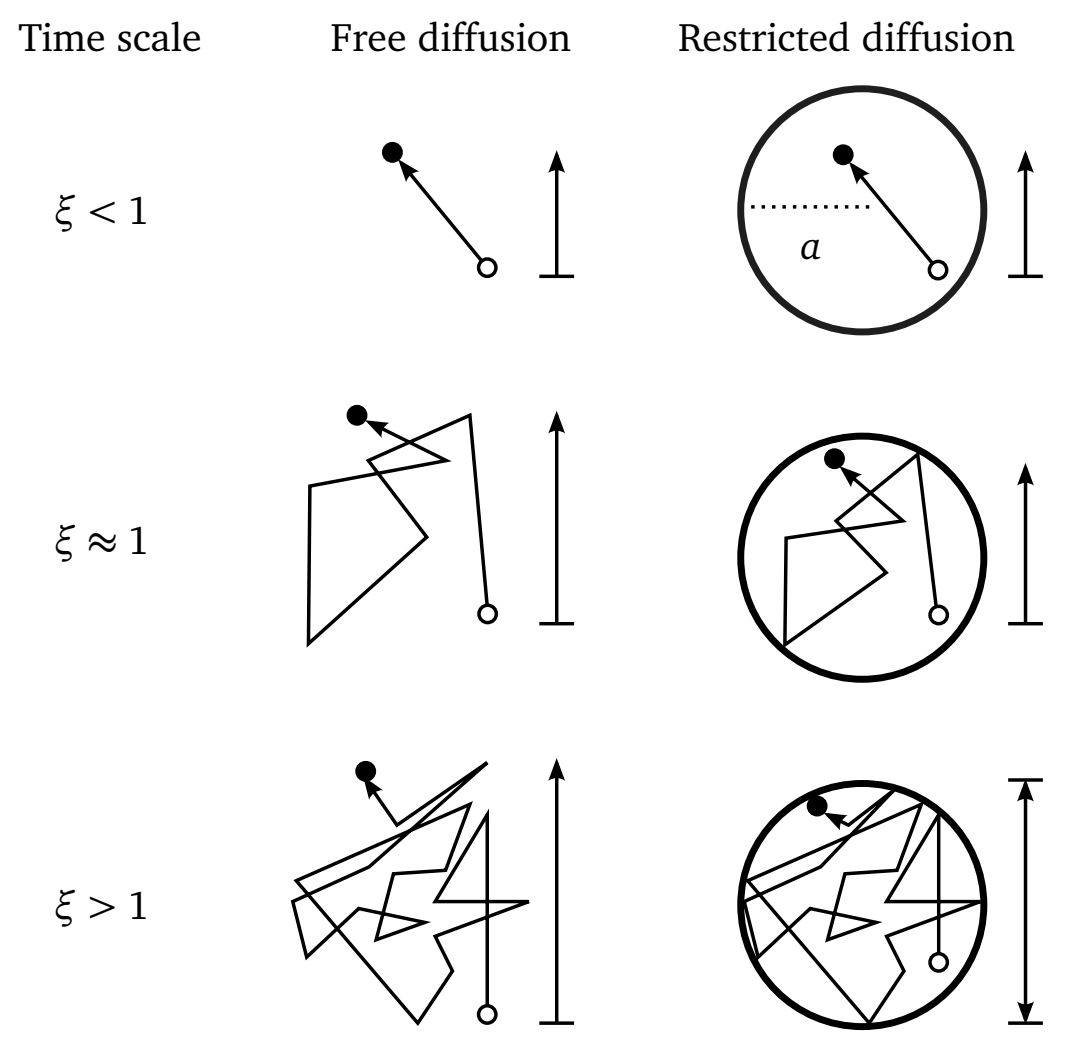

Figure 3.2: A graphical depiction of the three modes of diffusion within a restricting geometry.

\subsection{Diffusion and magnetic field gradients}

This section will detail the use of NMR experiments to measure self-diffusion and consequently obtain information about the microstructure of a heterogeneous system such as an emulsion.

\subsubsection{Magnetic field gradients as spatial labels}

Spins in a spatially homogeneous magnetic field $\mathbf{B}_{0}$ will precess at the same Larmor frequency $\omega_{0}$ regardless of their position $\mathbf{r}$,

$$
\omega_{0}=\gamma B_{0},
$$


where $\gamma$ is the gyromagnetic ratio of the nucleus under observation and a single quantum transition is assumed. However, if we impose a homogeneous magnetic field gradient $\mathbf{g}$, on the sample, defined as

$$
\mathbf{g}=\nabla \mathbf{B}_{0}=\frac{\partial \mathbf{B}_{z}}{\partial x} \hat{\mathbf{x}}+\frac{\partial \mathbf{B}_{z}}{\partial y} \hat{\mathbf{y}}+\frac{\partial \mathbf{B}_{z}}{\partial z} \hat{\mathbf{z}}
$$

the magnetic field is no longer spatially homogeneous. Instead, the strength of the magnetic field at any point is now given by

$$
B(\mathbf{r})=B_{0}+\mathbf{g} \cdot \mathbf{r} .
$$

As a result, the Larmor frequency of a spin is now dependent on its position within in the sample

$$
\omega(\mathbf{r})=\omega_{0}+\gamma(\mathbf{g} \cdot \mathbf{r}) .
$$

The Larmor frequency can therefore be used as a spatial label with respect to the direction of the gradient, which is the basis for measuring diffusion using NMR.

To emphasise the concept of spatial labelling, if a spin at $\mathbf{r}$ is subjected to a rectangular gradient of duration $\delta$, the phase difference is given by

$$
\phi(\mathbf{r})=\gamma \delta(\mathbf{g} \cdot \mathbf{r})
$$

That is, the phase of the spin is shifted by an amount that is dependent on both the dephasing strength of the gradient $(\gamma \delta g)$ and its position or motion in the direction of the gradient. Should the spin then diffuse to $\mathbf{r}^{\prime}$ and then be subjected to a second gradient pulse, the phase change is then

$$
\Delta \phi=\gamma \delta \mathbf{g} \cdot\left(\mathbf{r}^{\prime}-\mathbf{r}\right) .
$$

The signal attenuation due to the distribution of $\phi$ is [50]

$$
E(t)=\int_{-\infty}^{\infty} U_{\phi}(\phi, t) \exp (i \phi) d \phi,
$$

where $U_{\phi}(\phi, t)$ is the phase-distribution function. If the spins do no diffuse between the two gradient pulses, then there is no distribution of phases and consequently no signal attenuation. Conversely, diffusion will induce a distribution of phases and attenuate the signal. The greater the extent of diffusion, the weaker the signal.

It is therefore possible to correlate the signal strength with the magnitude of molecular self-diffusion. The following sections will detail the experimental and theoretical techniques for performing this analysis.

\subsubsection{Measuring diffusion with magnetic field gradients}

\subsubsection{Spin echoes}

Most NMR experiments that measure molecular self-diffusion are based on the concept of a spin echo [51]. Developed in 1950 by Hahn [52], a spin echo is an experimentally measurable signal generated by the refocusing of spin magnetisation. Hahn achieved this via application of a $\pi / 2_{x}$ pulse to tip the magnetisation onto the $x-y$ plane. After 
a time period $\tau$, a $\pi_{x}$ pulse is applied to refocus the dephasing spins and produce an echo at $2 \tau$ (figure 3.3 ).

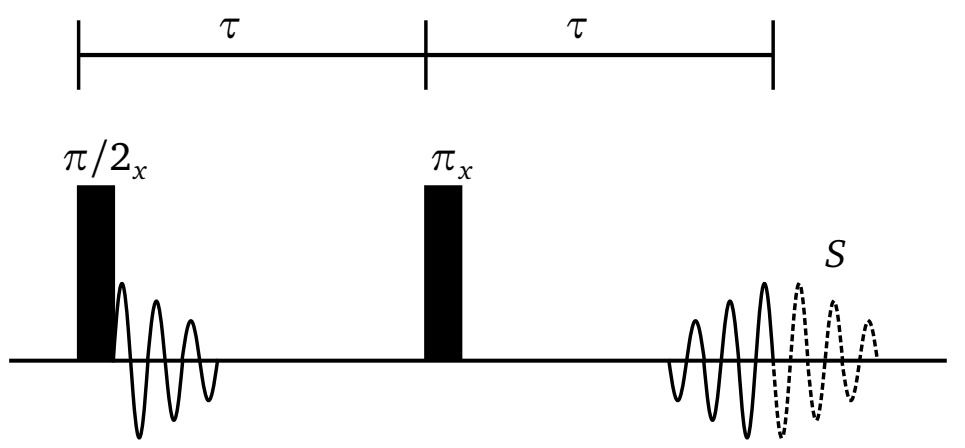

Figure 3.3: The Hahn spin echo pulse sequence. The time between the initial $\pi / 2_{x}$ pulse and the refocusing $\pi$ pulse is equal to the time elapsed between the refocusing pulse and the observed spin echo $-\tau$. The second half of the echo $S$, indicated by dotted lines, is digitised and used as the free induction decay. The notation $\pi_{x}$ indicates a pulse of $\pi$ radians about the $x$-axis.

The spin echo pulse sequence was modified by Hahn [52] as well as Carr and Purcell [50] to include a constant background gradient. While the constant gradient made it possible to accurately measure diffusion coefficients of pure liquids, it introduced several experimental problems [53]. Because the gradient is on during acquisition, the linewidth of the spectrum is broadened which means it is not possible to determine the diffusion coefficient of multiple components simultaneously. The broadening is increased as the gradient strength is increased, exacerbating this problem for systems with small diffusion coefficients. Additionally, the time over which diffusion is measured is not well defined, reducing its applicability to systems with restricted diffusion. Also, it is not possible to separate the effects of spin-spin relaxation and diffusion.

Pulsed gradients eliminate these effects meaning multi-component systems and samples with very small diffusion coefficients can be measured reliably. As a result, contemporary pulse sequences use pulsed gradients.

\subsubsection{The pulsed gradient spin echo experiment}

Discovered in 1965 by Stejskal and Tanner [53], the pulsed gradient spin echo (PGSE) experiment is an extension of the Hahn spin echo experiment with two gradient pulses of length $\delta$ and magnitude $g$ (figure 3.4).

Initially the magnetisation is tipped onto the $x-y$ plane with an excitation $2 \pi / 2_{x}$ pulse. At a time $t_{1}$ the first gradient pulse is applied which spatially encodes the spins with a Larmor frequency $\omega(\mathbf{r})$. After $\tau$, the magnetisation is refocused with a $\pi_{x}$ pulse. When the diffusion time $\Delta$ has elapsed, the second gradient of effective opposite polarity is applied to spatially decode the spins. Those molecules that have not diffused during $\Delta$ refocus completely and produce the maximum signal. Conversely, molecules that have diffused do not refocus completely and produce an attenuation of the signal which is contained in the spin echo produced at $2 \tau$. The signal for free diffusion is equal to

$$
S(g, 2 \tau)=M_{0} \exp \left(-\frac{2 \tau}{T_{2}}\right) \exp \left(-\gamma^{2} g^{2} D \delta^{2}\left(\Delta-\frac{\delta}{3}\right)\right)
$$



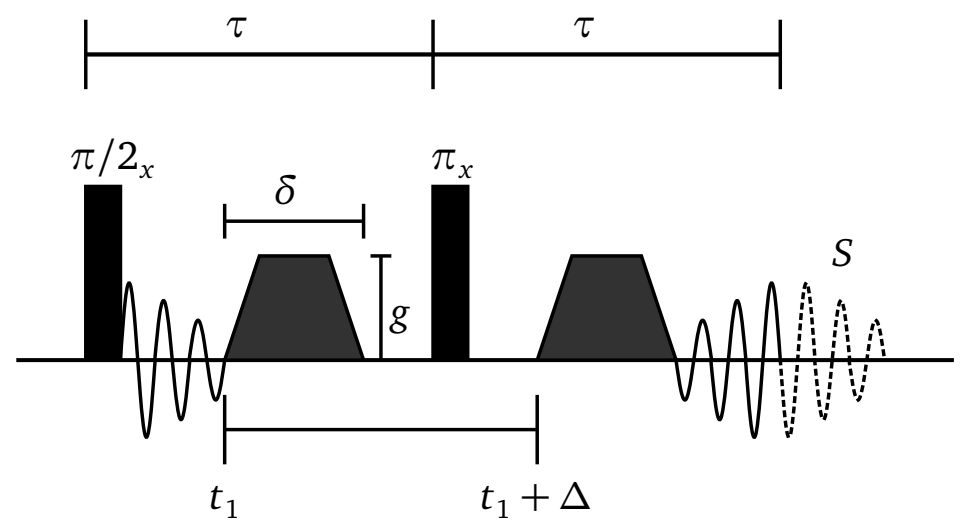

Figure 3.4: A depiction of the PGSE pulse sequence. The second portion of the spin echo S, is used as the free induction decay.

where $M_{0}$ is the initial bulk magnetisation and $T_{2}$ the spin-spin relaxation time. Generally, the signal is normalised with respect to the echo intensity at $g=0$, which leads to the Stejskal-Tanner equation

$$
E(g, \Delta)=E(g, 2 \tau)=\frac{S(g, 2 \tau)}{S(g=0,2 \tau)}=\exp \left(-\gamma^{2} g^{2} D \delta^{2}\left(\Delta-\frac{\delta}{3}\right)\right)
$$

where $E$ is the echo attenuation due to diffusion. In a PGSE experiment, diffusion can be probed by varying $g, \delta$ or $\Delta$ and observing the effects on signal attenuation.

\subsubsection{The stimulated echo experiment}

In the PGSE experiment, the magnetisation is stored in the $x-y$ plane and the maximum value of delta is limited by the spin-spin relaxation time $T_{2}$. In many systems such as emulsions, the spin-lattice relaxation time $T_{1}$ is longer than $T_{2}$, which led to the development of the stimulated echo pulse sequence where the magnetisation is stored on the $z$-axis $[54,55]$.

As shown in figure 3.5, the initial excitation pulse is followed by the encoding gradient pulse at $t_{1}$. After $\tau_{1}$ has elapsed, the magnetisation is stored on the $z$-axis by a $\pi / 2_{x}$ pulse. During $\tau_{2}$, any residual magnetisation is removed by using a homospoil and at $\tau_{1}+\tau_{2}$ the magnetisation is brought back into the $x-y$ plane by a third $\pi / 2_{x}$ pulse. After $\Delta$ has elapsed, the second gradient pulse is applied to spatially decode the spins. Like the PGSE experiment, any attenuation of the signal is contained in the spin echo which is used as the FID.

The signal intensity of the spin echo for free diffusion is given by

$$
S(g, 2 \tau)=\frac{M_{0}}{2} \exp \left(-\frac{2 \tau_{1}}{T_{2}}-\frac{\tau_{2}}{T_{1}}\right) \exp \left(-\gamma^{2} g^{2} D \delta^{2}\left(\Delta-\frac{\delta}{3}\right)\right),
$$

and the expression for the signal attenuation is identical to equation 3.18. The $M_{0} / 2$ term arises because the second $\pi / 2_{x}$ pulse only stores half the magnetisation. It is preferable to use a STE over a PGSE when the ratio $T_{1} / T_{2}$ is greater than unity where the improved relaxation properties outweigh the loss of magnetisation [54]. 


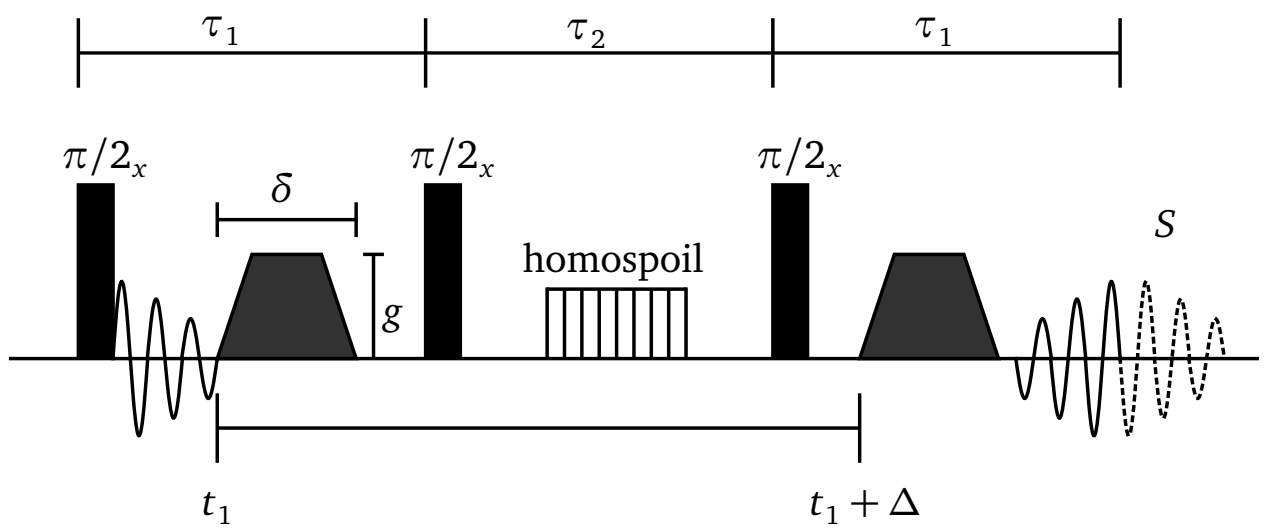

Figure 3.5: The STE pulse sequence. The second half of the stimulated echo is digitised (indicated by dots) and used as the FID. Any transverse magnetisation is purged using a homospoil so the phase cycle can be shortened.

\subsubsection{Correlating signal attenuation with diffusion}

There are three main theoretical routes to correlating signal attenuation with molecular self-diffusion [56]: solving the Bloch-Torrey equations [57], the Gaussian phase distribution (GPD) approximation [58] and the short gradient pulse (SGP) approximation $[53,59]$. All three methods produce identical results for free diffusion: equations 3.17 and 3.18 for the PGSE pulse sequence and equation 3.19 when using a stimulated echo [56]. However, once boundaries to diffusion are introduced, the solutions provided by the GPD and SGP models are divergent and solving the Bloch-Torrey equations becomes mathematically intractable [56]. For that reason, the Bloch-Torrey equations will not be discussed further.

\subsubsection{Free diffusion}

The GPD model is based on the assumption that when spins are subjected to a magnetic field gradient, the distribution of phases that is accumulated is Gaussian. That is,

$$
U_{\phi}(\phi, \Delta)=\left(2 \pi\left\langle\phi^{2}\right\rangle\right)^{-1 / 2} \exp \left(\frac{-\phi^{2}}{2\left\langle\phi^{2}\right\rangle}\right) .
$$

Using equation 3.16, the signal attenuation due to the Gaussian distribution of phases is

$$
E(\Delta)=\exp \left(\frac{-\left\langle\phi^{2}\right\rangle}{2}\right),
$$

and the mean-squared distribution of phases is [58]

$$
\left\langle\phi^{2}\right\rangle=\gamma^{2} g^{2} 2 \delta^{2}\left(\Delta-\frac{\delta}{3}\right) .
$$

By combining equations 3.20 and 3.22, the expression for the signal attenuation due to free diffusion is equation 3.18

$$
E(g, \Delta)=\exp \left(-\gamma^{2} g^{2} D \delta^{2}\left(\Delta-\frac{\delta}{3}\right)\right),
$$


where the $\Delta-\delta / 3$ term accounts for the finite width of the gradient pulse.

The SGP approximation assumes that the there is no motion during the application of a gradient pulse $[53,59]$. In essence, the gradient pulse is treated as a delta function. This is only valid experimentally when $\delta \ll \Delta$ and the distance diffused during the gradient pulse is much shorter than the characteristic dimension of the system [60].

The $q$-space formalism is typically used when discussing the SGP approximation, where $\mathbf{q}$ is given by [61]

$$
\mathbf{q}=\frac{1}{2 \pi} \gamma \delta \mathbf{g}
$$

Using this notation, the signal attenuation due to diffusion in the SGP limit is $[53,59]$

$$
E(\mathbf{q}, \Delta)=\iint \rho(\mathbf{r}) P_{s}\left(\mathbf{r} \mid \mathbf{r}^{\prime}, \Delta\right) \exp \left(i 2 \pi \mathbf{q} \cdot\left(\mathbf{r}^{\prime}-\mathbf{r}\right)\right) d \mathbf{r} d \mathbf{r}^{\prime},
$$

where the total signal is a superposition of signals in which each phase term is weighted by the probability of a spin diffusing from $\mathbf{r}$ to $\mathbf{r}^{\prime}$ during $\Delta$. By substituting the propagator for free diffusion (equation 3.6) into equation 3.24 and evaluating, the expression for signal attenuation due to free diffusion is

$$
E(\mathbf{q}, \Delta)=\exp \left(-\gamma^{2} g^{2} D \delta^{2} \Delta\right)=\exp \left(-(2 \pi q)^{2} D \Delta\right)
$$

which is equal to equation 3.18 in the limit of $\delta \rightarrow 0$.

\subsubsection{Restricted diffusion}

When $\xi>1$ and the mean-square displacement is no longer proportional to $\Delta$, the models developed for free diffusion will no longer correctly predict the signal attenuation. For extremely complex geometries, analytical solutions are not possible [62]. However, for simple geometries such as spheres, both the GPD and SGP approximations provide analytical solutions.

Assuming the spins are confined within a perfectly reflecting sphere of radius $a$, the expression for the signal attenuation using the GPD approximation is [63]

$$
\begin{aligned}
& E(g, \Delta)= \\
& \exp \left(-\frac{2 \gamma^{2} g^{2}}{D^{2}} \sum_{n=1}^{\infty} \frac{2 \alpha_{n}^{2} D \delta-2+2 L(\delta)-L(\Delta-\delta)+2 L(\Delta)-L(\Delta+\delta)}{\alpha_{n}^{6}\left(a^{2} \alpha_{n}^{2}-2\right)}\right),
\end{aligned}
$$

where $L(t)=\exp \left(-\alpha_{n}^{2} D t\right)$ and $\alpha_{n}$ are the roots of the equation

$$
\left(\alpha_{n} a\right) J_{3 / 2}^{\prime}\left(\alpha_{n} a\right)-(1 / 2) J_{3 / 2}\left(\alpha_{n} a\right)=0,
$$

where $J$ is the Bessel function of the first kind. The complexity of equation 3.26 emphasises the difficulties associated with correlating signal attenuation and diffusion for restricting geometries.

In the experimental conditions where the SGP approximation is valid $(\Delta \rightarrow \infty)$, the following expression for attenuation due to diffusion within a reflecting sphere is obtained

$$
E(q, \infty)=\frac{9[(2 \pi q a) \cos (2 \pi q a)-\sin (2 \pi q a)]^{2}}{(2 \pi q a)^{6}}
$$


The significant difference between the two models is a result of diffusive diffraction that is accounted for by the SGP approximation. At short diffusion times, the probability of displacement by $\mathbf{R}\left(\mathbf{r}^{\prime}-\mathbf{r}\right)$ is dependent on the starting position and displacements to new positions are strongly correlated with the initial spin density $\rho(\mathbf{r})$. If $\Delta \rightarrow \infty$ and therefore $\Delta \gg a^{2} / D$, then most molecules will collide with the restricting boundary and the probability of displacement by $\mathbf{R}$ is independent of the starting position. Consequently, $P_{s}\left(\mathbf{r} \mid \mathbf{r}^{\prime}, \infty\right)$ reduces to $\rho\left(\mathbf{r}^{\prime}\right)$ [64] and the average propagator becomes

$$
\overline{P_{s}}(\mathbf{R}, \infty)=\int \rho(\mathbf{r}) \rho(\mathbf{r}+\mathbf{R}) d \mathbf{r} .
$$

Therefore $\overline{P_{s}}(\mathbf{R}, \infty)$ is the autocorrelation function of the molecular density. Because the Fourier transform of a time autocorrelation function is the frequency power spectrum, $E(\mathbf{q}, \infty)$ is the power spectrum of $\rho(\mathbf{r})[65]$ :

$$
\begin{aligned}
E(\mathbf{q}, \infty) & =\iint \rho(\mathbf{r}) \rho(\mathbf{r}+\mathbf{R}) \exp (i 2 \pi \mathbf{q} \cdot \mathbf{R}) d \mathbf{r} d \mathbf{R} \\
& =|S(\mathbf{q})|^{2},
\end{aligned}
$$

where $S(\mathbf{q})$ is the Fourier transform of $\rho\left(\mathbf{r}^{\prime}\right)$. Equation 3.29 shows the origin of diffraction-like effects in diffusion NMR $[65,66]$. In neutron scattering, $|S(\mathbf{q})|^{2}$ is the structure factor - the mathematical description of how a material scatters radiation. Structural information is obtained directly from a plot of the signal attenuation as a function of $\mathbf{q}$, where minima in the plot correspond to characteristic distances in the confining geometry [60].

\subsubsection{Validity of the models}

The validity of the GPD and SGP approximations was tested by Balinov et al. [67] using simulations of Brownian motion. It was found that for diffusion within a reflecting sphere, the GPD model was valid for $\xi<1, \xi \approx 1$ and $\xi>1$. In contrast, the SGP model was valid only for $\xi>1$ and small gradient strengths. Despite this, the SGP approximation is able to predict diffraction-like effects in diffusion NMR experiments, while the GPD model cannot [68].

\subsection{Emulsion characterisation}

Nuclear magnetic resonance has been used to characterise emulsions since 1968 [59]. By correlating the signal attenuation with an assumed form of the emulsion droplet size distribution, it is possible to obtain the mean droplet size as well as the standard deviation [69]. A particular advantage of using NMR to characterise emulsions is that the sample does not need to be diluted or have the refractive index of the phases matched [70], meaning the sample can be analysed "as-is" without the need for preparative work.

\subsubsection{Polydisperse emulsions}

The expressions for diffusion within a reflecting sphere discussed earlier are only suitable for monodisperse systems. For a conventional emulsion that is composed of polydisperse droplets with an unknown distribution of droplet radii $P(a)$, the signal 
attenuation due to diffusion of the dispersed phase is given by [71]

$$
E(\mathbf{q}, \Delta)=\frac{\int_{0}^{\infty} a^{3} P(a) E(\mathbf{q}, \Delta) d a}{\int_{0}^{\infty} a^{3} P(a) d a},
$$

where $E(\mathbf{q}, \Delta)$ is the attenuation function for a reflecting sphere with radius $a$. Equation 3.30 contributions to the attenuation from the droplets is weighted by the volume of each droplet. The significant problem with this expression is that $P(a)$ is often not known, and a form of the distribution is often assumed. Packer and Rees suggested a log-normal distribution was suitable for most emulsions produced with conventional techniques [71], although other distributions have been used. Callaghan et al. assumed a normal distribution when determining the droplet size distribution of cheese [72].

It is possible to obtain $P(a)$ from the form of the signal attenuation. Ambrosone and coworkers developed a method to invert equation 3.30 and approximate $P(a)$ using a generating function [73, 74]. Additionally, Hollingsworth and Johns [75] developed a method for inverting 3.30 using regularisation methods.

\subsubsection{Monodisperse emulsions}

The GPD and SGP models for diffusion within a reflecting sphere are applicable to monodisperse emulsions with little modification. Woodward et al. [76] characterised a series of monodisperse poly(dimethyl siloxane)-in-water emulsions using the STE pulse sequence and diffusive diffraction of the dispersed phase.

As $\Delta$ was increased, minima in the $q$-space plots began to develop that were shallower than predicted by equation 3.27 (figure 3.6). This was attributed to the fact that not all of the dispersed phase molecules were experiencing restricted diffusion. To fit the attenuation data and extract the size of the droplets, a simple model was developed that combined the expressions for free diffusion (equation 3.19) and restricted diffusion within a reflecting sphere (equation 3.27)

$$
E_{\text {free+restricted }}=(1-x) E_{\text {free }}+x E_{\text {restricted }}
$$

where $x$ is the proportion of molecules experiencing restricted diffusion. This approach was similar to that used by Fourel et al. when characterising polydisperse emulsions[77]. Figure 3.6 shows that equation 3.31 was a satisfactory fit for the attenuation data below high values of $q$ for all $\Delta$. Because the position of the first minimum is sufficient to calculate the size of the restricting geometry, this simple model produced values for the droplet sizes that were concordant with confocal microscopy and laser diffraction.

\subsubsection{Non-NMR methods}

There are several additional methods that are routinely used to obtain information about the droplet size and size distribution of emulsions and other colloidal systems. These include confocal and optical microscopy [78], light scattering [4] and ultrasound spectroscopy [79]. The best technique is often dictated by the nature of the sample and often multiple techniques will be used to ensure the results are meaningful. The advantage of using NMR, particularly with concentrated samples, it that does not require sample dilution to obtain meaningful data. In fact, concentrated emulsions may 


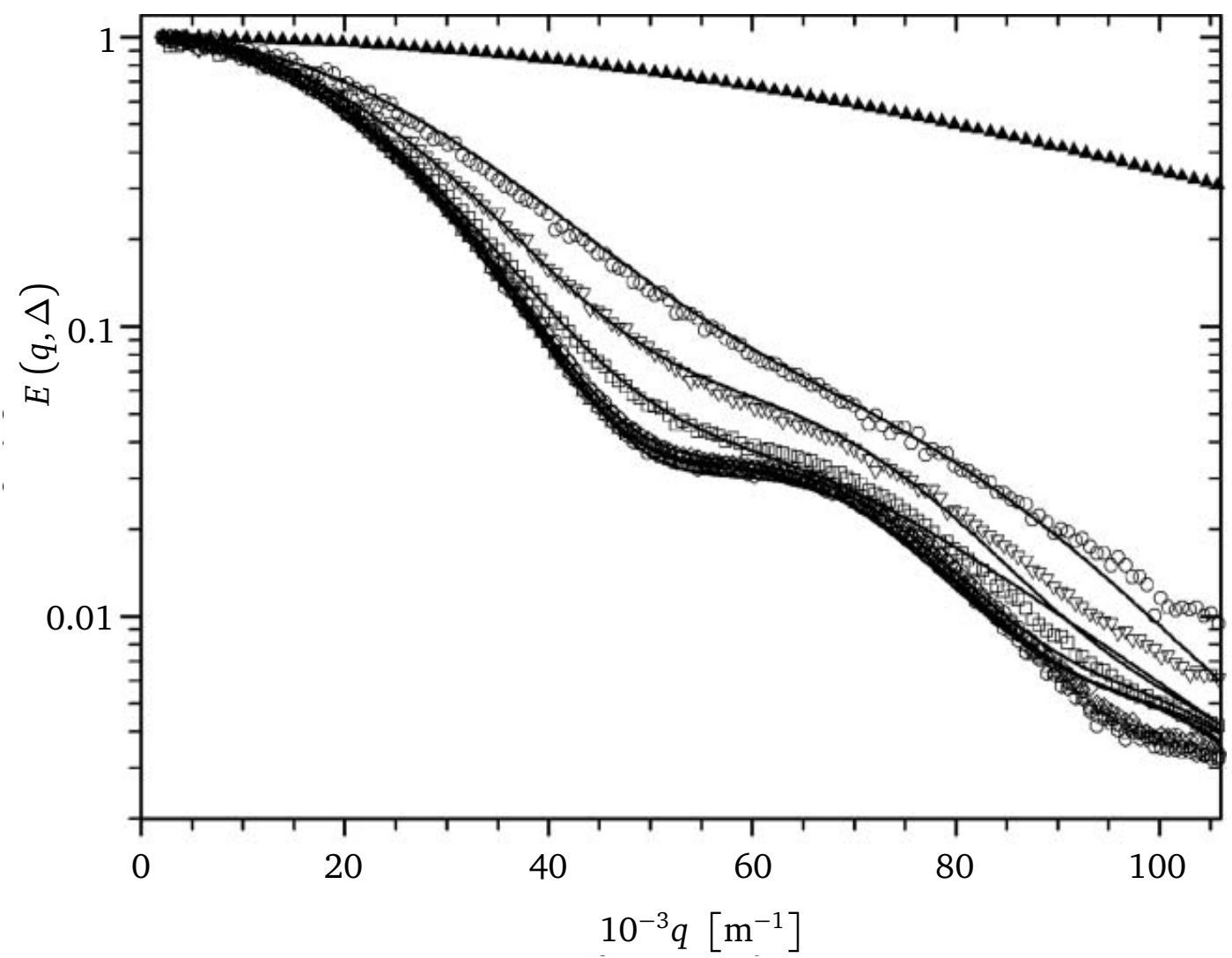

Figure 3.6: Various q-space plots at different diffusion times between 20 and $2500 \mathrm{~ms}$ for an emulsion with $2 a=27 \mu \mathrm{m}: \boldsymbol{\Delta}=20 \mathrm{~ms}, \bigcirc=310 \mathrm{~ms}, \nabla=490 \mathrm{~ms}$ and $\square=770 \mathrm{~ms}$. The remaining plots cover the range 1280 to $2500 \mathrm{~ms}$ and are indistinguishable. The solid lines represent a fit to equation 3.31. Reproduced from [76].

be advantageous if dispersed phase diffusion is being measured. For many emulsions, including those used in this research program, dilution or the vigorous stirring that is used in some equipment can limit the information that can be obtained (such as the nature of the droplet packing, for example), force the sample into a different phase or even destroy it. For these reasons, NMR was chosen as the primary technique for droplet size and size distribution analysis with optical microscopy used for verification. 

Everything flows

and nothing abides;

everything gives way

and nothing stays fixed.

Heraclitus (535-475 BCE)

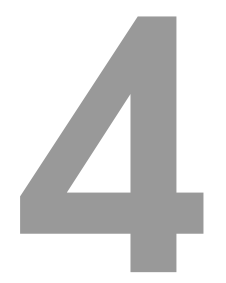

\section{An introduction to rheology}

Simply put, rheology is the study of deformation and flow of matter with the ultimate goal of linking the bulk flow properties of the sample with its microstructure. In the rheological study of emulsions, flow is typically induced by the application of shear (tangential) forces using geometries that oscillate or continuously rotate. In this chapter, the fundamental concepts of rheology pertinent to emulsions will be discussed and a review existing material in the field presented. Much of the theoretical work has been by drawn from the texts written by Larson [80], Macosko [81] and Morrison [82], and the interested reader is encouraged to consult these references for a more general treatment of what is a highly diverse field.

\subsection{Hookean solids and Newtonian fluids}

Hookean solids and Newtonian fluids represent limiting behaviours of materials at opposite ends of the spectrum: fluids flow and solids do not. The simplicity of the models allows for a straightforward introduction to stress and strain, two of the most fundamental concepts in rheology.

\subsubsection{Hookean solids}

The stress $\sigma$, on a material is defined as the magnitude of the applied force $F$, acting over an area $A$

$$
\sigma=\frac{F}{A}
$$

The force can have components that are normal or tangential to the surface of the material, which produces extensional/commpressive stress and shear stress, respectively. The applied stress will deform the material, and this deformation is known as the strain $\gamma$, on the material. It is defined as the amount of deformation $\Delta l$, relative to a reference configuration length, area or volume $l$ :

$$
\gamma=\frac{\Delta l}{l}
$$

Strain is often measured in units of per cent, where 100\% represents a deformation equal in magnitude to the orginal configuration. Graphical depictions of stress and strain are shown in figure 4.1.

The application of stress on a Hookean solid will induce an instantaneous and propor- 
tional strain response. This can be depicted mathematically as

$$
\sigma=G \gamma
$$

where $G$ is the shear, or rigidity, modulus and is directly analagous to the spring constant $k$, in Hooke's law $F=k x$, in that it is a measure of how "stiff" the material is. If the applied stress is constant, the solid will remain deformed until the stress subsides, at which point the solid returns to its original configuration. This is often termed elastic behaviour.

\subsubsection{Newtonian fluids}

If a fluid is placed between two parallel plates and sheared by moving one plate at a velocity $u$, a velocity gradient is created through the sample, as shown in figure 4.1c. The velocity gradient is given by $u / d$, where $d$ is the distance between the plates, and indicates how quickly the "parts" (as phrased by Newton) of the fluid are separated from each other due to the deformation. This can also be described by the shear rate (or strain rate) $\dot{\gamma}$, which is defined as

$$
\dot{\gamma}=\frac{\partial \gamma}{\partial t}
$$

where $t$ is time. Newtonian fluids have a shear stress response to strain that is proportional to the shear rate. The proportionality constant is the fluid viscosity $\eta$,

(a)

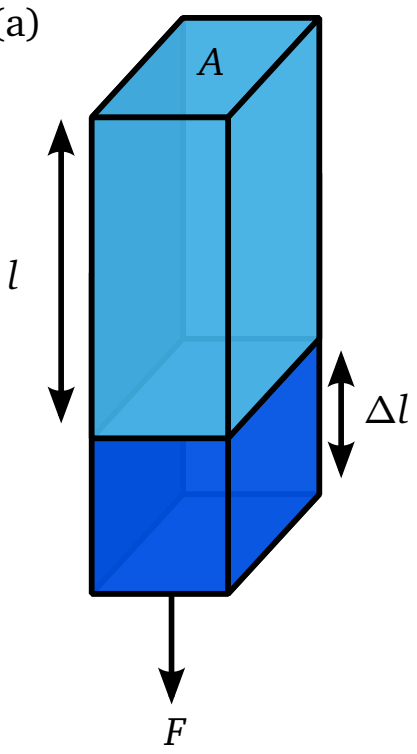

(b)

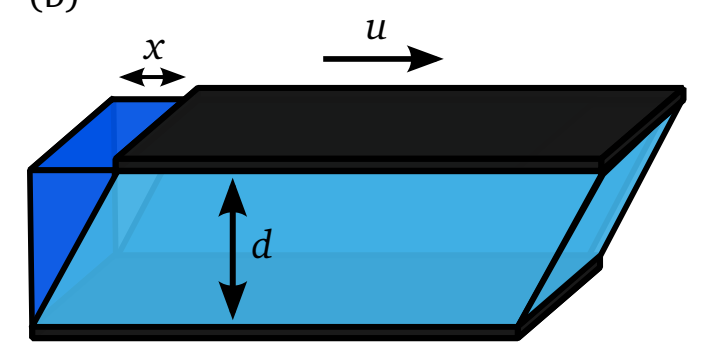

(c)

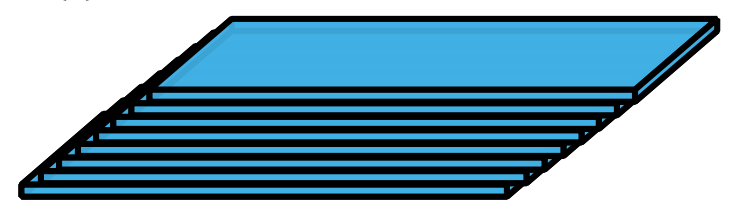

Figure 4.1: Visual depictions of stress and strain: (a) shows an extensional stress inducing strain; (b) shows two parallel plates in relative motion inducing strain equal to $x / d$, where $x$ is the displacement; (c) shows how the velocity gradient in shear flow can be visualised as a series of parallel planes in motion that move apart at $\dot{\gamma}-$ the "parts" referred to by Newton. 
which, measures the resistance to the applied force that produces a deformation

$$
\sigma=\eta \dot{\gamma}
$$

A Newtonian fluid deforms instantly when a constant stress is applied at a rate $\dot{\gamma}$ and continues to do so until the stress is removed, at which point none of the deformation is recovered. Materials that behave in such a way are often called viscous.

Equations 4.3 and 4.5 are both examples of constitutive relations. That is, they link the stress response of a material to the applied strain.

\subsection{Increased complexity}

\subsubsection{Stress and strain in multiple dimensions}

Stress and strain in real samples occurs in three dimensions. In addition to shear forces there are also normal forces (forces perpendicular to a surface) that play an important role in certain soft matter systems such as polymers [83, 84]. To deal with this increased complexity in an elegant way, tensors are used to represent stresses and strains. Tensors can be considered as an extension of the concept of scalars, vectors and matrices.

The stress tensor $\boldsymbol{\sigma}$, is a matrix that contains information about the stresses acting on a sample in three dimensions:

$$
\boldsymbol{\sigma}=\left(\begin{array}{lll}
\sigma_{11} & \sigma_{12} & \sigma_{13} \\
\sigma_{21} & \sigma_{22} & \sigma_{23} \\
\sigma_{31} & \sigma_{32} & \sigma_{33}
\end{array}\right) .
$$

Each element $\sigma_{i j}$ describes the stress acting perpendicular to the $i$ th face in the $j$ th

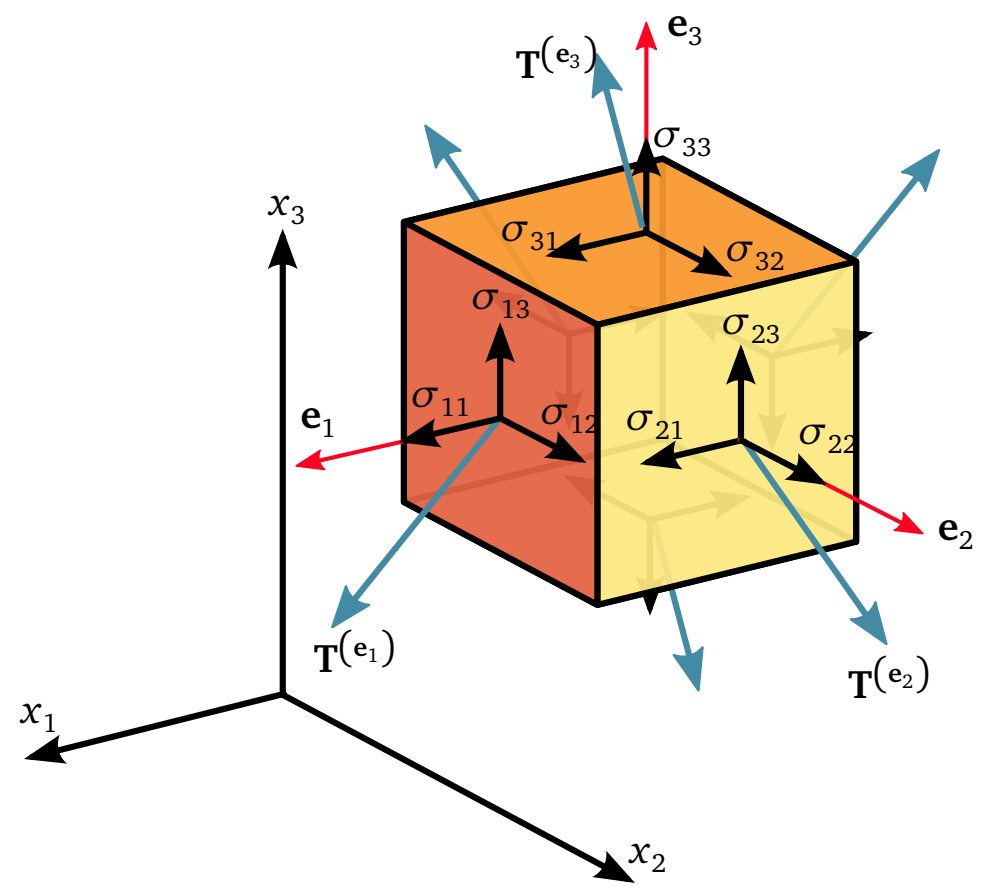

Figure 4.2: A graphical depiction of each element of the stress tensor in cartesian coordinates. The vector $\mathbf{e}_{a}$ is the normal vector for face $a$ and $\mathbf{T}^{\left(\mathbf{e}_{a}\right)}$ is the total stress on face $a$. 
direction. Shear stresses are described by elements with $i \neq j$ and normal stresses by elements with $i=j$. A graphical depiction of the stress tensor elements is given in figure 4.2 .

Strain can be depicted tensorially with knowledge of the velocity gradient tensor $\nabla \mathbf{v}$, which describes the magnitude of the velocity variation in each direction. Using the same coordinates as in figure 4.2 , the velocity gradient is given by

$$
\nabla \mathbf{v}=\left(\begin{array}{lll}
\frac{\partial v_{1}}{\partial x_{1}} & \frac{\partial v_{1}}{\partial x_{2}} & \frac{\partial v_{1}}{\partial x_{3}} \\
\frac{\partial v_{2}}{\partial x_{1}} & \frac{\partial v_{2}}{\partial x_{2}} & \frac{\partial v_{2}}{\partial x_{3}} \\
\frac{\partial v_{3}}{\partial x_{1}} & \frac{\partial v_{3}}{\partial x_{2}} & \frac{\partial v_{3}}{\partial x_{3}}
\end{array}\right)
$$

The shear rate tensor $\dot{\gamma}$, is then defined as

$$
\dot{\gamma} \equiv \nabla \mathbf{v}+(\nabla \mathbf{v})^{T}
$$

where $(\nabla \mathbf{v})^{T}$ is the transpose of the velocity gradient tensor. Examples of $\dot{\gamma}$ are given for rheological flows in section 4.3.3.1.

\subsubsection{Non-Newtonian fluids}

Contrary to Newtonian fluids, many materials in the real world exhibit viscosities that are a function of the applied shear rate. Examples include polymer melts, foods such as yogurt and most multiphase materials such as emulsions and dispersions [85]. As such, equation 4.5 no longer applies and instead we have

$$
\sigma=\eta(\dot{\gamma}) \dot{\gamma}
$$

which is the constitutive equation for non-Newtonian materials [86] and the basis for most phenomenological models of non-Newtonian behaviour [87], some of which are discussed below.

There are several broad classifications that are used to describe non-Newtonian materials. Materials that show a decrease in viscosity as the shear rate increases are classified as shear-thinning. This behaviour is common in systems where the molecules or particles can align in a way that reduces the chance of entaglement or obstruction $[88,89]$. In emulsions, an increase in shear rate can induce a decrease in viscosity by disrupting the packing of droplets and deforming the droplets so that they are elongated and aligned in the direction of of the shearing [90, 91].

Certain materials can show an increase in viscosity as the shear rate increases. They are classified as shear thickening. Materials that develop a microstructure that is highly associated, such as some polymeric systems, are likely to show shear-thickening behaviour [92]. However, it is claimed that all materials show some degree of shear thickening at shear rates that are beyond those practical in rheological experiments [93].

Shear thinning and shear thickening as well as Newtonian behaviour can be modelled using a simple power law developed by Ostwald and de Waele [94]

$$
\sigma=m \dot{\gamma}^{n}
$$


where $n$ is the flow behaviour index and the viscosity is replaced by $m$, the consistency coefficient. Shear thinning and shear thickening materials have $n<1$ and $n>1$, respectively. If $n=0$ the material is Newtonian and $m=\eta$. In some cases materials such as mayonnaise appear to behave elastically until a critical amount of stress, called the yield stress, is applied. Equation 4.10 can be modified to include a yield stress $\sigma_{y}$, giving the Herschel-Bulkley model [95]

$$
\sigma=\sigma_{y}+m \dot{\gamma}^{n}
$$

Model flow curves are given for shear thinning, shear thickening and yield stress materials in figure 4.3 .

While the concept of yield stresses can be useful when modelling materials, there is strong debate as to whether yield stresses truly exist [96]. Newer rheological equipment is currently capable of achieving shear rates as low as $10^{-6} \mathrm{~s}^{-1}$, several orders of magnitude lower than when the concept of yield stress was first modeled. As such, Barnes [97] suggested that a yield stress only represented what could not be measured.

There is a variety of other phenomenological and empirical models for non-Newtonian behaviour. In most cases, the validity of a model is restricted to the classes of materials for which it was developed. Several examples are given in references [98] and [99].

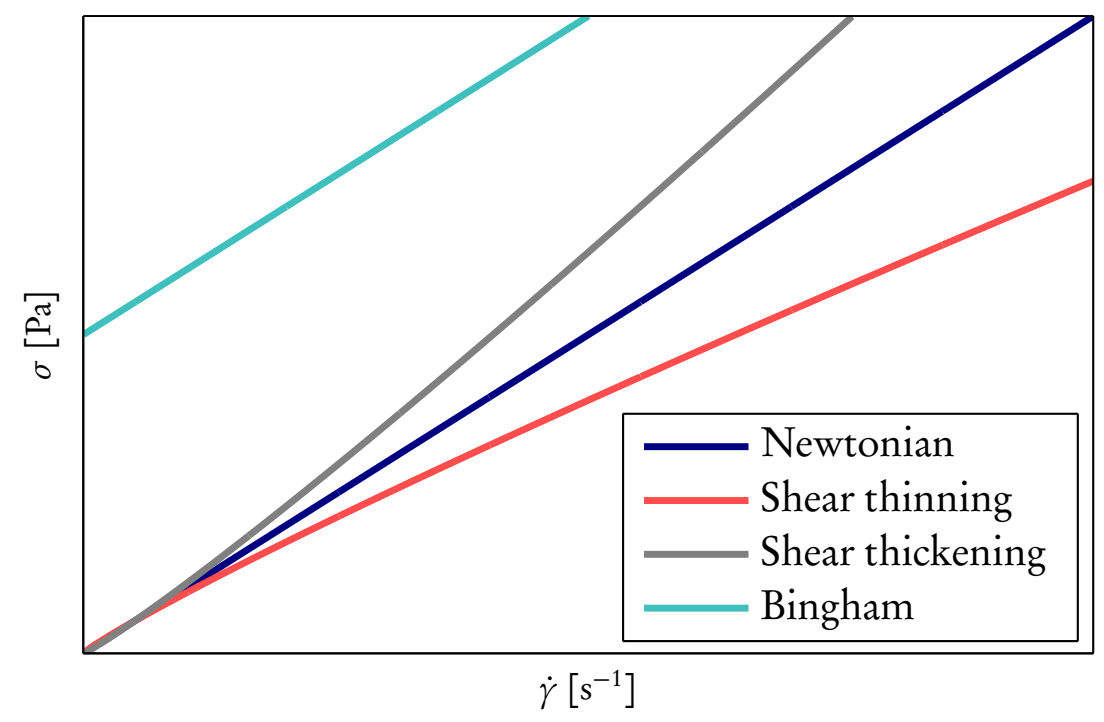

Figure 4.3: A rheogram comparing Newtonain, non-Newtonian and yield stress behaviours discussed in the text. Bingham yield behaviour is a special case of the Herschel-Bulkley model (equation 4.11 where the material flows in a Newtonian manner after exceeding the yield stress.

\subsubsection{Viscoelasticity}

Elastic and viscous responses represent limiting cases for the flow properties of a material, and often a combination of the two behaviours is observed. For example, after applying a constant stress to an unknown sample, a portion of the material's deformation may be restored. That is, the material has a "memory" of its deformational history. Such materials are viscoelastic. Whether we observe a viscous, elastic or 
viscoelastic response is dependent on the characteristic time of the material's relaxation and for how long the material is observed. In rheology, the Deborah number is often used to establish which type of flow behaviour will be observed. Using the variables mentioned above, the Deborah number De, is defined to be [100]

$$
\operatorname{De} \equiv \frac{\lambda}{t}
$$

where $\lambda$ is the relaxation time of the material and $t$ is the observation time. If the observation time is long or the relaxation time is short, De $<1$ and the material appears to flow. Conversely, if the observation time is short and the relaxation time is long, De $>1$ and the material behaves as a Hookean solid. For De $\sim 1$ the material will appear to be viscoelastic.

\subsubsection{The Maxwell model}

Proposed by James Clerk Maxwell in 1867 [101], the empirical Maxwell model includes viscous and elastic components in the form of a dashpot with viscosity $\eta$, and a spring with elastic modulus $G$, connected in series (figure 4.4). An elongational force $f$ will cause the spring to produce a restoring force opposing the deformation while the piston in the dashpot experiences a drag while moving through the liquid. Because the two elements are connected in series, the displacement, or strain, experienced by the dashpot and spring is additive. This gives

$$
\boldsymbol{\sigma}+\lambda \frac{\partial \boldsymbol{\sigma}}{\partial t}=\eta \dot{\gamma}
$$

where $\lambda=\eta / G$ is the relaxation time of the material and defines how quickly stress is acquired and lost as a strain is applied and stopped, respectively. The additive nature is apparent if we consider a constant stress, where $\partial \sigma / \partial t \rightarrow 0$ and Newton's law is produced. Conversely, over short time periods, $\partial \boldsymbol{\sigma} / \partial t$ becomes much larger than $\sigma$, which can then be ignored, producing Hooke's law.

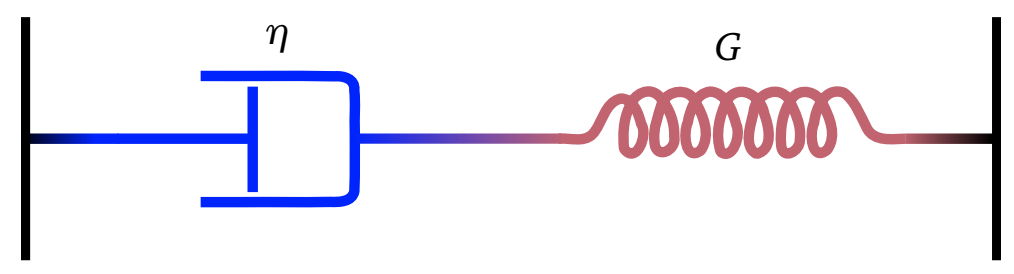

Figure 4.4: A schematic depiction of the Maxwell model, where a dashpot with viscosity $\eta$ (blue), and a spring with elastic modulus $G$ (red), are connected in series.

Where Maxwell's model differs from those given in previous sections, is that it accounts for the deformational history of the material. This is more apparent if equation 4.13 is solved for $\boldsymbol{\sigma}$ to give

$$
\boldsymbol{\sigma}(t)=\int_{-\infty}^{t}\left[\frac{\eta_{0}}{\lambda} \exp \left(\frac{-\left(t-t^{\prime}\right)}{\lambda}\right)\right] \dot{\gamma}\left(t^{\prime}\right) d t^{\prime},
$$

where $\eta_{0}$ is the zero shear viscosity, and $t^{\prime}$ is a dummy variable for integration. In the Maxwell model stress is a function of the history of the shear rate. The portion of the 
equation inside the square brackets is a decay function that acts as a weighting on the shear rate tensor, such that the farther back in time the deformation occurred, the less impact it has on the stress at time $t$.

Materials may have multiple relaxation times, in which case the Maxwell model can be extended to include an arbitrary number of relaxation times:

$$
\boldsymbol{\sigma}(t)=\int_{-\infty}^{t}\left[\sum_{k=1}^{n} \frac{\eta_{k}}{\lambda_{k}} \exp \left(\frac{-\left(t-t^{\prime}\right)}{\lambda_{k}}\right)\right] \dot{\gamma}\left(t^{\prime}\right) d t^{\prime},
$$

which is known as the generalised Maxwell model. This leads to the idea of a generalised viscoelastic fluid, where the portion of the expression within the square brackets is replaced with any function that is found to describe the strain history of the sample.

There are many other models that incorporate viscous and elastic elements connected in a variety of ways. One example is the Kelvin-Voigt model, where a dashpot and spring are connected in parallel, rather than in series [102]. The use of one model over another is justified only if it more accurately predicts the viscoelastic behaviour of a sample. Indeed, a significant problem with such models is that they offer no link between the structure of a material and its properties.

These models are also limited to the linear viscoelastic range of the material, where the stress response and applied strain are directly proportional. This is an approximation that typically holds when the deformation is small compared to the dimensions of the sample $(\sim 1 \%)$ [103]. The theories developed to account for the non-linear behaviour of materials at large strains are more complicated than the Maxwell model and similar approaches.

\subsection{Rheometry}

Rheometry is the experimental technique used to probe the flow properties of a material. Using a rheometer, the sample is typically deformed by a geometry controlled by an electric motor that is able to produce a variety of flows appropriate for measuring non-Newtonian and viscoelastic responses. This section will cover the types of rheometers, geometries and flows that are commonly used to investigate emulsion rheology.

\subsubsection{Rheometers}

There are two main types of rotational rheometers: controlled shear stress and controlled shear strain. Schematic depictions of both are given in figure 4.5. In controlled shear stress instruments the motor that drives the measuring geometry and the transducer that measures the response of the fluid are mounted on the same side of the sample. Either the tourque $\mathscr{T}$, is set by the user and the rotational speed of the geometry $\Omega$, is measured by a position sensor, or the rotational speed is set and the resulting torque exerted by the sample is measured.

Controlled shear strain rheometers typically have the motor and transducer mounted on opposite sides of the sample. The angular velocity or defelction angle $\varphi$, of the motor is set, and the force exerted on the measuring geometry by the sample is measured. The advantage of this system is that it reduces the influence of the measuring geometry inertia on the results. This can be particularly important at high rotational speeds or 
(a)

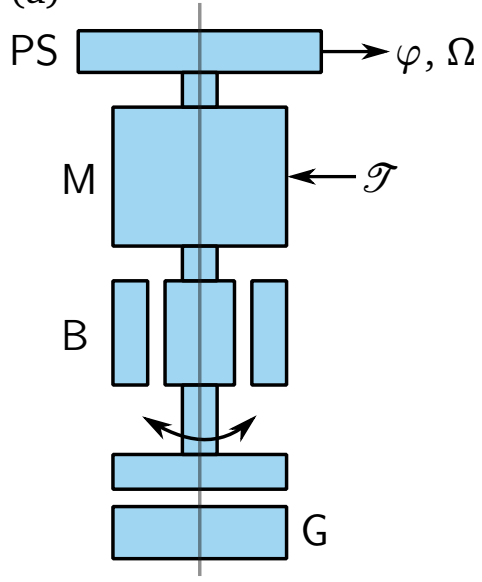

(b)

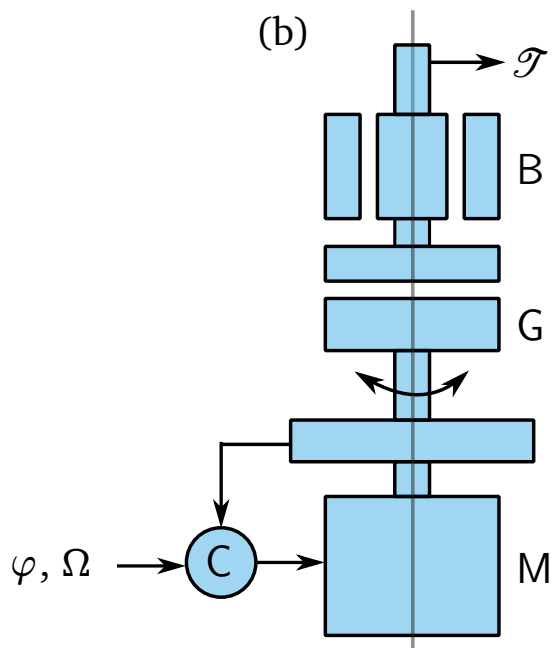

Figure 4.5: Schematic depictions of controlled shear stress (a) and controlled shear strain (b) rheometers. Controlled stress rheometers use a torque setting $(\mathscr{T})$ supplied to the motor $(\mathrm{M})$, which drives the measuring geometry (G). The flow properties of the sample cause the geometry to rotate at an angular velocity $\Omega$ that is measured by the position sensor (PS). Controlled shear strain rheometers drive the base of the measuring geometry using a set angular velocity or deflection angle (C), and the torque exerted by the sample is measured. Both systems use an air bearing to reduce friction (B).

when probing low viscosity materials [104]. With modern equipment it often possible to obtain similar test results regardless of the type of system used.

\subsubsection{Measuring geometries}

There are several measuring geometries commonly used to produce shear flow, with each performing optimally with different types of samples. Three common geometries used for rotational and oscillatory experiments: parallel plates, cone and plate, and concentric cylinders are shown in figure 4.6 and discussed below.

\subsubsection{Parallel plates}

The parallel plate measuring geometry involves a disk of radius $R$, rotating with an angular velocity $\Omega$, separated from a stationary plate by a distance $H$ (figure 4.6(a)). In most cases $H \ll R$. When the angular velocity is constant, the sample only moves in the direction of the rotation $\theta$, giving the following velocity field

$$
\mathbf{v}=\left(\begin{array}{c}
0 \\
v_{\theta} \\
0
\end{array}\right)
$$

The velocity of the sample is zero at the stationary plate $(z=0)$ and increases to a maximum value at the rotating disk

$$
v_{\theta}=\frac{r \Omega z}{H},
$$

which can be substituted into equation 4.8 to give an expression for the spatial dependence of the shear rate:

$$
\dot{\gamma}=\frac{r \Omega}{H} .
$$




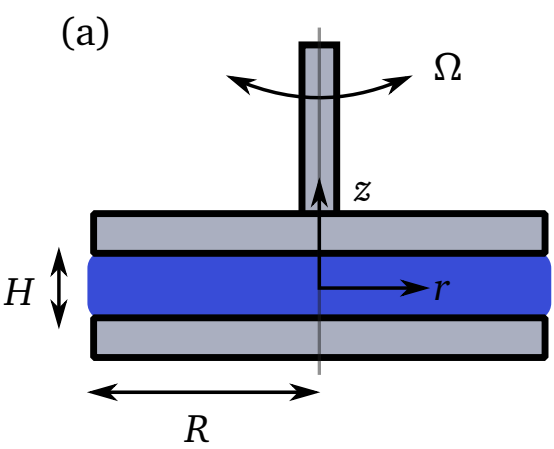

$(\theta$-plane section)

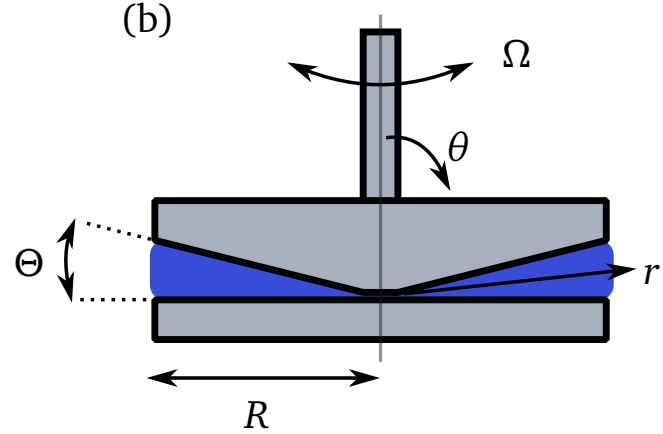

( $\phi$-plane section)

(c)

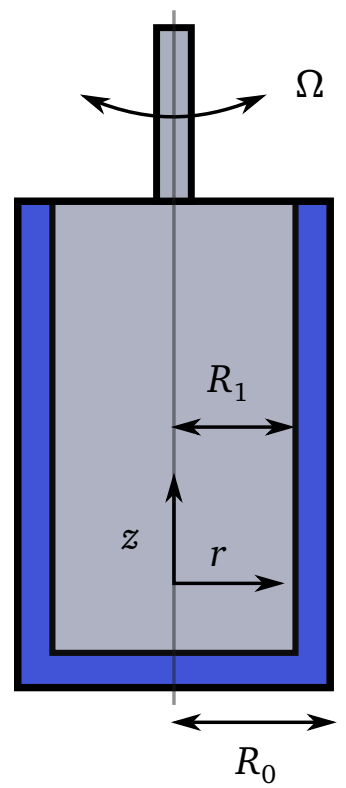

$(\theta$-plane section)

Figure 4.6: Geometries commonly used in rotational and oscillatory rheometry: parallel plates (a), cone and plate (b) and concentric cylinders (c).

From this equation, the greatest limitation of the parallel plate measuring system is evident: the shear rate varies with radial position, with a maximum at $r=R$. This is often accounted for in rheological software by displaying an average value of the shear rate, or reporting the maximum shear rate at the edge.

Despite this limitation, parallel plates are commonly used when investigating suspensions or performing experiments at varied temperatures on sample of moderate to high viscosity. Unlike the cone and plate and concentric cylinder geometries discussed below, the gap can be set as large as the sample will allow to accommodate particles becoming jammed or distorted. A large gap may produce a temperature gradient across the sample which can be mitigated by allowing the material to equilibrate before starting a measurement.

\subsubsection{Cone and plate}

The cone and plate geometry uses a similar setup to the parallel plate geometry, where the disk is replaced with a cone (figure 4.6b). The use of a cone with a small angle $\Theta$, creates a homogeneous flow with a constant shear rate across the sample. In most 
cases, $\Theta$ is between 1 and $4^{\circ}$. Using spherical coordinates, the velocity field created by a cone rotating at a constant angular velocity is

$$
\mathbf{v}=\left(\begin{array}{c}
0 \\
0 \\
v_{\phi}
\end{array}\right)
$$

The velocity at the stationary base is zero and $r \Omega$ at the cone, which gives the following expression for the velocity:

$$
v_{\phi}=\frac{r \Omega}{\Theta}\left(\frac{\pi}{2}-1\right),
$$

which can be substituted into equation 4.8 to give the shear rate applied to the sample

$$
\dot{\gamma}=\frac{\Omega}{\Theta} .
$$

The constant shear rate across the sample means that the stress is simply calculated from the bulk torque exerted on the geometry by the sample.

Cone and plate geometries are suitable for a range of non-particular materials of moderate to high viscosity and require a small amount of sample. Materials such as suspensions and emulsions must have a small mean particle or droplet size to produce reliable results. While temperature gradients may be present during a variable temperature experiment, as with parallel plates, this can be mitigated by allowing the sample to equilibrate.

\subsubsection{Concentric cylinders}

The concentric cylinder measuring geometry consists a smaller cylinder with radius $R_{1}$, often called the "bob", suspended within a larger cylinder of radius $R_{0}$, often called the "cup". Most commercial rheometers use the Searle method, where the bob is rotated and the cup remains stationary. If the bob rotates at a constant angular velocity, in cylindrical coordinates the velocity field is

$$
\mathbf{v}=\left(\begin{array}{c}
0 \\
v_{\theta} \\
0
\end{array}\right)
$$

The velocity is zero at the cup wall and increases to a maximum of $R_{1} \Omega$ at the surface of the bob to giving a velocity profile of

$$
v_{\theta}=\frac{\Omega R_{1}^{2}}{R_{0}^{2}-R_{1}^{2}}\left(\frac{R_{0}^{2}}{r}-r\right) .
$$

Using equation 4.8 , the average shear rate across the gap between the two cylinder is

$$
\dot{\gamma}=\frac{\Omega R_{1}}{R_{0}-R_{1}} .
$$

Concentric cylinders are suitable for low viscosity samples because of the large surface area that is in contact with the sample. Because the gap between the cylinders must be small to assume a linear velocity profile, concentric cylinders are not appropriate for 
particulate materials. Concentric cylinders also require large sample volumes, typically greater than $10 \mathrm{~cm}^{3}$.

\subsubsection{Standard flows and material functions}

The information we can obtain from a rheological experiment about a material is dependent on the type of flow to which the material is subjected. The following sections will cover the flows employed to determine the non-Newtonian and viscoelastic properties of monodisperse emulsions.

\subsubsection{Simple shear flow: flow curves}

Simple shear flow was described briefly in section 4.1.2 as two parallel plates in relative motion at a shear rate $\dot{\gamma}$. By varying the shear rate and measuring the associated stress response, we are able to probe the non-Newtonian and non-linear properties of a material. Simple shear flow has the following velocity profile:

$$
\mathbf{v}=\left(\begin{array}{l}
v_{1} \\
v_{2} \\
v_{3}
\end{array}\right)=\left(\begin{array}{c}
\dot{\gamma}_{21}(t) x_{2} \\
0 \\
0
\end{array}\right)
$$

which is depicted in figure 4.7. Using equation 4.8 , this velocity profile produces the following shear rate tensor

$$
\dot{\gamma}=\left(\begin{array}{ccc}
0 & \dot{\gamma}_{21}(t) & 0 \\
\dot{\gamma}_{21}(t) & 0 & 0 \\
0 & 0 & 0
\end{array}\right) \text { where } \dot{\gamma}_{21}=\frac{\partial v_{1}}{\partial x_{2}}
$$

The shear rate is independent of position, making simple shear a homogeneous flow. As indicated by $\dot{\gamma}_{21}(t)$, homogeneous flows can be time variant. Simple shear flow is only achieved approximately when the gap is narrow.

A flow curve is a rheogram where shear stress is plotted as a function of shear rate and is a common way to present the flow properties of a material under steady shear (see figure 4.3. When measuring a flow curve, the shear rate is typically varied in a linear ramp or preferably as a series of steps where the shear rate is held constant for a set period of time ("peak holds"). The latter approach displays how the stress response varies with time and allows the material to reach a steady state if one exists (for example, see [105]).

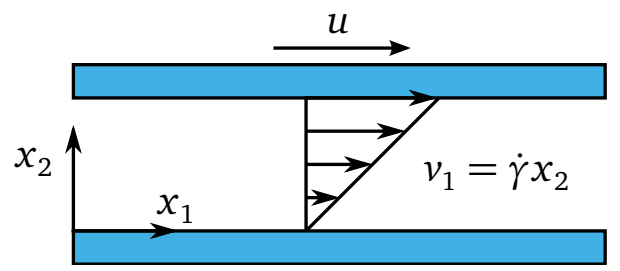

Figure 4.7: A depiction of simple shear in the $x_{3}$ plane that shows the velocity gradient across the sample at a constant shear rate.

\subsubsection{Small amplitude oscillatory shear: frequency and strain sweeps}

The linear viscoelastic properties of an emulsion can be examined by applying a small oscillatory deformation and measuring the stress response. Oscillatory shear is an 
unsteady flow with the following periodic velocity profile:

$$
\mathbf{v}=\left(\begin{array}{c}
\dot{\gamma}(t) x_{2} \\
0 \\
0
\end{array}\right) \text { where } \dot{\gamma}(t)=\gamma_{0} \cos \omega t
$$

The oscillation has an amplitude $\dot{\gamma}_{0}$ and frequency $\omega$. The strain on the material can be calculated by integrating the shear rate over the measurement time $t$

$$
\begin{aligned}
\gamma(t) & =\int_{0}^{t} \dot{\gamma}(t) d t^{\prime} \\
& =\int_{0}^{t} \dot{\gamma} \cos \omega t d t^{\prime} \\
& =\gamma_{0} \sin \omega t,
\end{aligned}
$$

where $\gamma_{0}=\dot{\gamma}_{0} / \omega$ is the strain amplitude. The stress response to the strain is also oscillatory

$$
\sigma(t)=\sigma_{0} \sin (\omega t+\delta),
$$

where $\sigma_{0}$ is the stress amplitude and $\delta$ is the phase lag - the strain and the associated stress response are not necessarily in phase. Equation 4.29 can be expanded using trigonometric identities to give

$$
\sigma(t)=\left(\sigma_{0} \cos \delta\right) \sin \omega t+\left(\sigma_{0} \sin \delta\right) \cos \omega t
$$

This shows that there is a portion of the stress response in phase with the strain $(\propto \sin \omega t)$ and a portion that is in phase with the strain rate $(\propto \cos \omega t)$. In other words, the material is viscoelastic.

To determine the proportion of viscous and elastic behaviour, two material functions are introduced: the storage modulus $G^{\prime}(\omega)$, and the loss modulus $G^{\prime \prime}(\omega)$ which are defined as

$$
G^{\prime}(\omega) \equiv \frac{\sigma_{0}}{\gamma_{0}} \cos \delta
$$

and

$$
G^{\prime \prime}(\omega) \equiv \frac{\sigma_{0}}{\gamma_{0}} \sin \delta
$$

The storage and loss moduli essentially show the degree of elastic and viscous response the material shows to oscillatory shear, respectively.

For a purely viscous fluid, the stress response is in phase with the strain rate $\left(\delta=90^{\circ}\right)$ and $G^{\prime}(\omega)=0$ while $G^{\prime \prime}(\omega)=\eta \omega$. For purely elastic solids, the stress response is in phase with the shear rate $(\delta=0)$ and $G^{\prime}(\omega)=G$ while $G^{\prime \prime}(\omega)=0$. Viscoelastic materials have nonzero values of the storage and loss moduli, which can vary significantly with the frequency of oscillation.

There are two main experiments that utilise oscillatory shear: frequency sweeps and strain sweeps. As the names suggest, a frequency sweep involves varying the oscillation frequency at a fixed strain, while a strain sweep involves varying the strain amplitude at a fixed oscillation frequency. When characterising a sample, several strain sweeps 
will be performed to identify the linear viscoelastic range of the material, ideally at the maximum and minimum frequencies that will be probed during the frequency sweep, as well as at least one somewhere between. The frequency sweep is then performed using a strain amplitude that falls in that range [104].

\subsection{Emulsion rheology}

Emulsions can exhibit a wide range of rheological properties that are primarily determined by composition, interfacial interactions and droplet microstructure $[8,106]$. The example given in chapter 1 of an emulsion changing from a viscous liquid at low $\phi$ to a material with a large elastic response at higher $\phi$ effectively illustrates the significant effect afforded by changing one parameter. Emulsions are complicated systems, despite this it is possible to make qualitative generalisations about how flow properties are influenced by certain variables, which will be attempted in the following sections.

\subsubsection{Droplet dynamics}

The viscoelastic properties of emulsions is intimately linked to the ability of the suspended droplets to pack and deform [107]. The shape of a droplet is governed by a balance between competing forces: the shear stress applied during a rheological experiment and the interfacial tension [80]. The shear stresses tend to stretch and elongate the droplet in the direction of the flow, while the interfacial tension and viscosity of the droplet oppose the elongation [108]. The influence of these effects is given by the capillary number and viscosity ratio first introduced in chapter 2

$$
\mathrm{Ca} \equiv \frac{\eta_{c} G \dot{\gamma}}{\xi}
$$

and

$$
p=\frac{\eta_{d}}{\eta_{c}}
$$

Taylor [109] quantified droplet deformation by introducing the deformation parameter

$$
D \equiv \frac{L-B}{L+B}
$$

where $L$ and $B$ are the lengths of the major and minor axes of the droplet with volume $(4 / 3) \pi L B^{2}$, respectively. The deformation parameter is zero for a spherical droplet and tends to 1 as the droplet becomes infinitely slender.

In terms of the capillary number and viscosity ratio, the deformation parameter is

$$
D=\mathrm{Ca}\left(\frac{19 p+16}{16(p+1)}\right)
$$

If $p<1$, low shear rates (typically below $1 \mathrm{~s}^{-1}$ ) cause the droplet to elongate and assume an elliptical shape with its long axis orientated $45^{\circ}$ to the direction of shear [110]. As the shear rate increases, the droplet is further elongated and its long axis rotates into the plane of shear. At higher shear rates, the droplet may deform further into long, thin threads until a critical shear rate is reached when the droplet will burst. This typically occurs when $D \approx 0.5$. For $p>1$ the degree of deformation is modest. 
At most the droplet will form a prolate ellipse with its long axis orientated in the direction of shear and no breakup is observed [80]. The deformable nature of droplets is responsible for the non-Newtonian and viscoelastic effects seen in the majority of emulsion systems, even when both phases are Newtonian liquids.

\subsubsection{Dilute emulsions}

Dilute emulsions are typically defined as those that have a volume fraction low enough that droplet-droplet interactions do not influence its rheological properties. They show shear thinning behaviour at all volume fractions and shear rates [7], and in their linear viscoelastic range have $G^{\prime}<G^{\prime \prime}$. As $\phi$ increases, the shear thinning becomes increasingly pronounced along with a raise in the $\eta_{0}$ as well as $G^{\prime}$ and $G^{\prime \prime}$, though they are still predominately liquid-like materials (for example, see [111] and [112]).

The first theoretical description of dilute emulsions was made by Taylor in 1932 [113], where he derived the following equation for the viscosity of an infinitely dilute emulsion at low $\mathrm{Ca}$

$$
\eta=1+\left(\frac{1+2.5 p}{1+p}\right) \phi,
$$

on the basis that there is no droplet deformation at low shear rates and that there are no droplet-droplet interactions. Equation 4.35 holds at very low volume fractions for viscosity ratios between 0.5 and 5, where droplet deformation is not significant [114].

Oldroyd [115] used an effective medium approach, where the emulsion was seen as a homogeneous medium, to relax the requirement of infinite dilution and assumed that the droplets deviated only slightly from their original shape. He obtained the following expression for the zero shear viscosity of a dilute emulsion

$$
\eta_{0}=\eta\left(\frac{10(p+1)+3 \phi(2+5 p)}{10(p+1)-2 \phi(2+5 p)}\right) .
$$

While deemed to be the most general solution for the viscosity of dilute emulsions [116], further generalisations have been made to predict the behaviour of emulsions with a wider range of droplet fractions with an increase in complexity (for example see [117] and [118]).

\subsubsection{Concentrated emulsions}

At high volume fractions, droplets are numerous enough that their properties such as deformability and packing have a significant effect on the bulk behaviour of the emulsion [116]. Like dilute emulsions, the viscosity of concentrated emulsions increases with volume fraction and as the droplet size decreases. Because the droplets are no longer free to move past each other unencumbered, concentrated emulsion tend to behave elastically at small oscillatory strains and low frequencies [119], although this can vary with droplet size [80].

At larger deformations, the droplets are able to elongate as described in section 4.4.1 to produce shear thinning behaviour, which becomes more pronounced as $\phi$ increases [120]. High volume fractions also induce thixotropic effects because the droplets are able to form a structure when they pack that is disrupted through an applied shear deformation and is restored some time after the deformation has ceased [121].

Attempts to construct theories that adequately describe concentrated emulsion have 
been hampered by the complexity of the systems. Pal [107] used a differential scheme originally described by Bruggeman [122] where a concentrated emulsion is obtained from the continuous phase by the addition of infinitesimal amounts of droplets. The equation for the viscosity of any concentrated emulsions is then

$$
\eta\left(\frac{M-P+32 \eta}{M-P+32}\right)^{N-1.25}\left(\frac{M-P+32}{M-P+32 \eta}\right)^{N+1.25}=(1-\phi)^{-2.5}
$$

where $M$ and $P$ are complicated variables that include the viscosity ratio and the shear rate dependence of the viscosity. This model accurately describes emulsion behaviour when $\phi<0.4$ [107]. Improvements to this model were made by accounting for the restrictions on droplet packing [107]. The right hand side of equation 4.37 was modified to be

$$
\left(1-\frac{\phi}{\phi_{m}}\right)^{-2.5 \phi_{m}}
$$

where $\phi_{m}$ is the maximum packing volume. This extension showed good agreement with experimental data across all volume fractions and capillary numbers measured. Despite the accuracy of the model, like others that are derived from similar principles it is complicated and unwieldy while offering little physical insight about the actual microstructure of the emulsion. Models derived from first principles are, however, complicated and in some cases lack generality (for example, see [123].

\subsubsection{Monodisperse emulsions}

Using the depletion flocculation technique, Mason et. al [10, 124, 125] as well as Chanamai and McClements [126, 127] have performed rheological studies on monodisperse emulsions.

At $\phi<0.4$, the monodisperse emulsions with droplet radii between 0.25 and $0.74 \mu \mathrm{m}$ behaved as monodisperse hard spheres at low shear rates [125, 128]. At higher volume fractions, the application of steady shear showed that the emulsions generally display shear thinning behaviour and the stress response increases significantly (six orders of magnitude) as $\phi$ increases from 0.55 to 0.87 . Above $\phi=0.58$, the emulsions exhibit a stress plateau that was attributed to the droplets being trapped in "cages" and being unable to relax to an unsheared state in a short time frame [125].

Oscillatory measurements showed that the emulsions were highly viscoelastic at strains below 0.1 , with $G^{\prime}$ two orders of magnitude larger than $G^{\prime \prime}$. Both moduli increased significantly with $\phi$ and as the droplet size decreased [124, 125, 127]. Frequency sweeps showed similar trends, with $G^{\prime}>G^{\prime \prime}$ and the moduli increasing with $\phi$ and as the droplet size decreased $[10,124]$. These trends were explained by suggesting higher volume fractions did not allow the droplets to relax to an undeformed state and that droplets are less able to deform as they decrease in size.

These studies did not investigate the effect of droplet size distribution on emulsion rheology, although it was suggested by Mason et al. [10] that monodisperse emulsions provide the ability to tailor the polydispersity of a sample. Currently, it is not quantitatively known how varying degrees of polydispersity affect the response of emulsions to steady and oscillatory shear. 



\section{Emulsion formation and structure}

Monodisperse emulsions were generated using microfluidic methods and characterised using PGSE NMR. In this chapter, the experimental details associated with emulsion formation and the subsequent characterisation are covered.

\subsection{Emulsion formation}

\subsubsection{Experimental details}

\subsubsection{The microfluidic device}

The microfluidic device used to generate monodisperse emulsions has an angled flow focusing geometry similar to those used by Woodward et al. [76]. The devices were fabricated by Epigem Limited from SU-8 that was treated with oxygen plasma prior to assembly. This rendered the internal surfaces hydrophilic to facilitate oil-in-water emulsion formation. An example device is shown in figure 5.1.

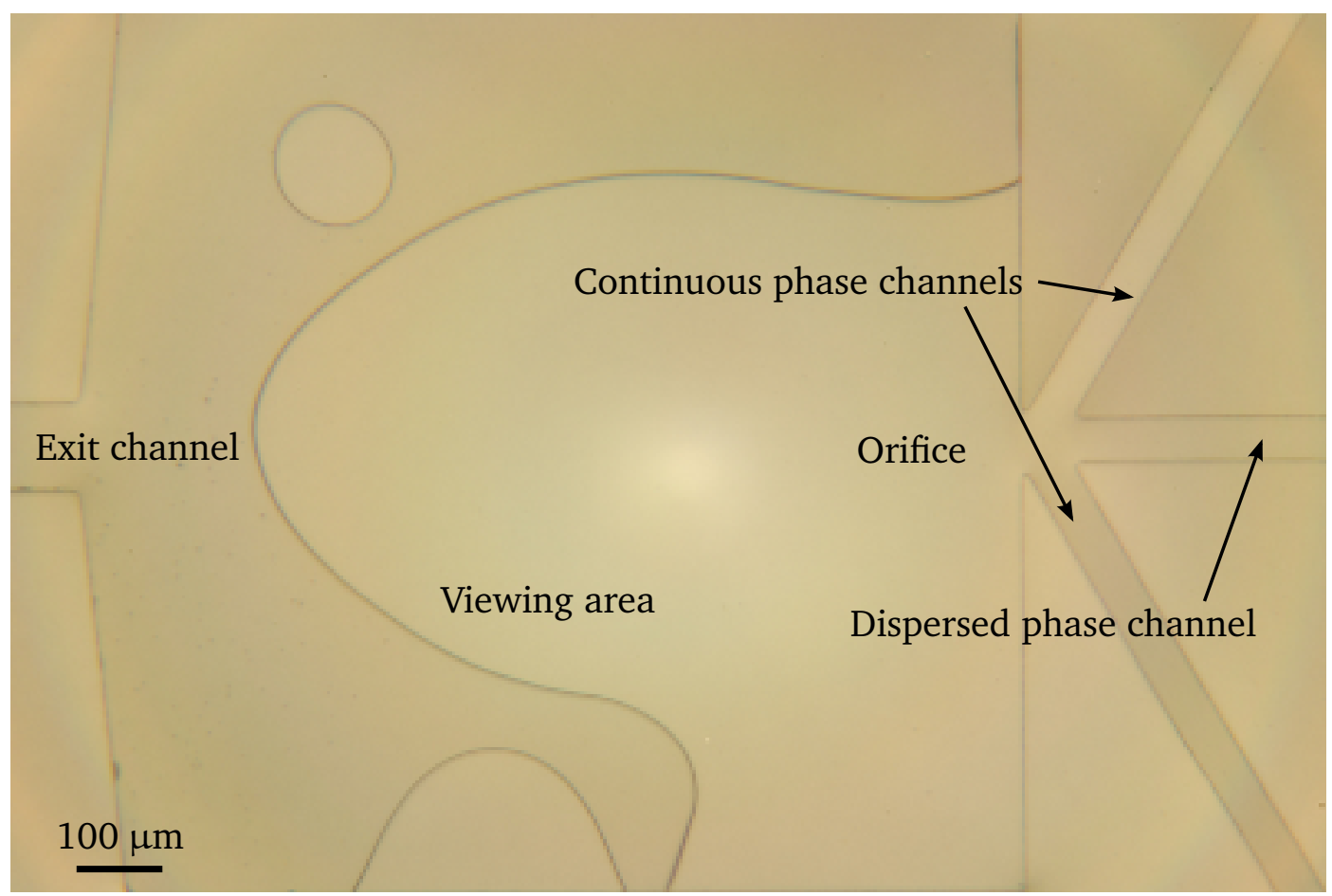

Figure 5.1: A micrograph of one of the flow focusing microfluidic devices used in this research.

The dispersed and continuous phases were introduced to the microfluidic device 
using one New Era Pump Systems NE-1000 syringe pump for each phase. Hamilton GASTIGHT $101010 \mathrm{~cm}^{3}$ and Norm-Ject polypropylene/polyethylene $50 \mathrm{~cm}^{3}$ syringes were used to house the dispersed and continuous phases, respectively. The solutions were filtered using $0.4 \mu \mathrm{m}$ pore size syringe filters. Using Luer-Lok connections, the syringes were interfaced with polytetrafluoroethylene tubing that connected to the microfluidic devices using the Epigem Fluence connection system. The emulsions were collected by running PTFE tubing from the exit channel to a large centrifuge tube that had been rinsed with the continuous phase, which prevented the rupturing of the droplets as they rolled down the wall of the tube.

\subsubsection{Materials}

The use of surface-modified SU-8 meant that only oil-in-water emulsions could be generated. Because the viscosity of water is approximately $1 \mathrm{mPas}$, it imparts a lower shear stress on the dispersed phase than the oils typically used in flow focusing experiments, where $\eta_{c}$ is typically greater than $40 \mathrm{mPa}$ (for example, see [31]). For this reason, n-dodecane (Sigma-Aldrich, $\geq 99 \%$, used as supplied) with $\eta_{d}=$ $1.344 \mathrm{mPas}$ [129] was chosen as the dispersed phase. The continuous phase was a 5 wt. \% solution of the polymeric surfactant Pluronic F-127 (Sigma, used as received) in deionised water (resistivity of $18.2 \mathrm{M} \Omega \mathrm{m}$ ). The use of a surfactant not only lowered the interfacial tension, facilitating smaller droplet sizes, but stablised the droplets against coalesence during formation and subsequent analysis.

\subsubsection{Results and discussion}

Each rheological analysis requires $\sim 1 \mathrm{~cm}^{3}$ of the creamed phase, hence low flow rates such as those used by Woodward et al. [76] $\left(Q_{d} \sim 30 \mu \mathrm{h}^{-1}, Q_{c} \sim 600 \mu \mathrm{h}^{-1}\right)$ to produce oil-in-water emulsions using a similar flow focusing geometry were deemed impractical. Instead, the dispersed phase flow rate was set at $1 \mathrm{~cm}^{3-1} \mathrm{~h}$ and the flow rate ratio was increased from 2.5 to 20 to obtain six emulsions with progressively smaller droplets, as shown in figure 5.2 and summarised in table 5.1. With this configuration, enough sample for one rheological test could be collected in one hour.

Table 5.1: The monodisperse emulsions and size as measured by optical microscopy.

\begin{tabular}{ccc}
\hline Emulsion name & Flow rate ratio & $\boldsymbol{a}_{\text {micro }}[\boldsymbol{\mu m}]$ \\
\hline A & 2.5 & $49 \pm 2$ \\
B & 5 & $44 \pm 1$ \\
C & 7.5 & $37 \pm 1$ \\
D & 10 & $33 \pm 2$ \\
E & 12.5 & $29 \pm 2$ \\
F & 20 & $20 \pm 2$ \\
\hline
\end{tabular}

For a wide range of flow rate ratios (from $\varphi=2.5$ to 15), the droplets form via a geometry controlled mechanism. Only once the flow rate ratio reached a value of 20 did the breakup mode change. This can be attributed to the relatively low viscosity of the continuous phase. Indeed, unlike most experimental systems used to study droplet formation in flow focusing systems (see [130]), the viscosity of the dispersed phase is greater than that of the continuous phase. As a result, the capillary number for the 

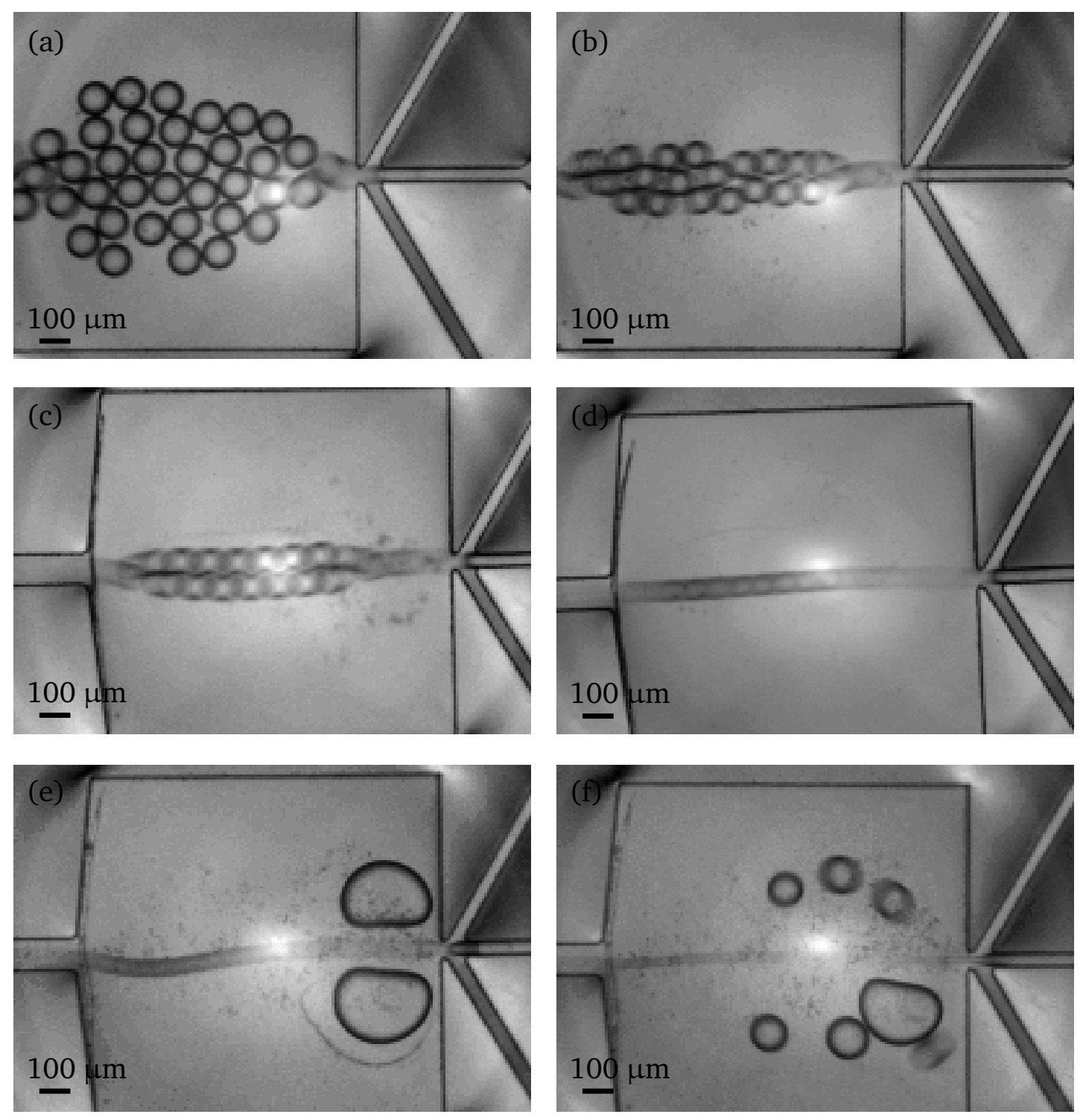

Figure 5.2: A comparison of the six emulsions produced, captured with a $1 / 500 \mathrm{~s}$ shutter speed. In (a), $\varphi=2.5$; in (b) $\varphi=5$; in (c) $\varphi=7.5$; in (d) $\varphi=10$; in (e) $\varphi=15$ and in (f) $\varphi=20$. As the continuous phase flow rate increases, the dispersed phase is thinned at the junction to produce a narrower stream of droplets at a higher formation frequency and eventually dripping sets in at $\varphi=20$. As can be seen in (e) and (f), at high flow rates large droplets of unknown origin become trapped within the viewing window.

system is low. That is, interfacial tension has more of an influence over the shape of the interface than the shearing from the continuous phase.

The balance of interfacial tension and viscous forces can be quantified using an expression for the capillary number during droplet breakup proposed by Anna and Mayer [31]

$$
\mathrm{Ca} \equiv \frac{\eta_{c} r Q_{c}}{\xi h \Delta z}\left(\frac{1}{W_{\text {or }}}-\frac{1}{2 W_{\text {up }}}\right),
$$

where $r$ is the half width of the dispersed phase channel, $h$ is the depth of the microfluidic device, $\Delta z$ is the distance between the continuous phase channel and the orifice, $W_{\text {or }}$ is the width of the orifice and $W_{\text {up }}$ is the width of the dispersed phase channel. The interfacial tension for the dodecane/Pluronic F127/water system was 


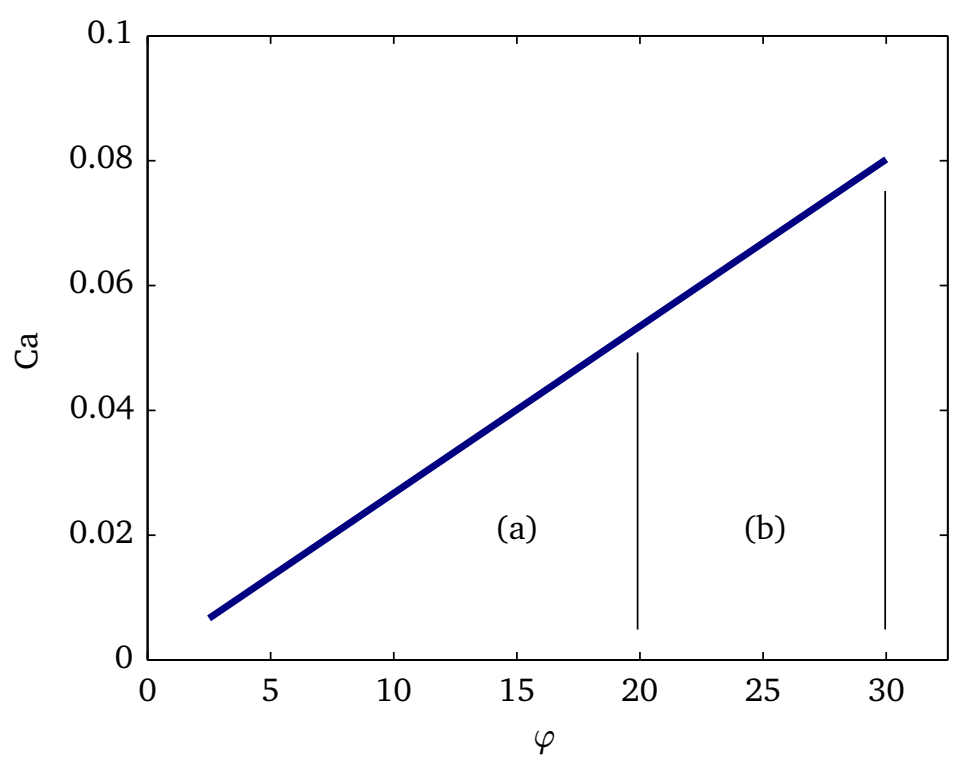

Figure 5.3: Ca for the observed droplet formation as predicted by equation 5.1, where $r=48 \mu \mathrm{m}$, $h=100 \mu \mathrm{m}, \Delta Z=67 \mu \mathrm{m}, W_{\text {or }}=48 \mu \mathrm{m}$ and $W_{\text {up }}=48 \mu \mathrm{m}$. Geometry controlled breakup was observed for flow rate ratios in region (a), while flow rate ratios in region (b) produced dripping. For $\varphi>30$, droplets formed in a highly erratic jetting mode.

measured as $8.3 \mathrm{mN} \mathrm{m}^{-1}$ at $5 \mathrm{wt}$ \% surfactant using a Wilhelmy plate. A plot of Ca as a function of flow rate ratio for the present system is given in figure 5.3.

The droplets form via a geometry controlled mechanism for $\mathrm{Ca}>0.05$, a dripping mechanism when $0.5<\mathrm{Ca}<0.08$ and a jetting mechanism for higher capillary numbers. This is significantly different to the values obtained by Lee et al. [130] using a flow focusing device with parallel input channels. In their system, the transition to tipstreaming occurred at $\mathrm{Ca} \approx 1$ and dripping was observed for $\mathrm{Ca}>3$.

This significant difference can be rationalised by considering the differences in the geometry of the two devices. The angled inputs of the microfluidic device used in the current research create a much smaller junction $(\Delta z=67 \mu \mathrm{m})$ than those found in parallel input devices (typically $\Delta z \geq 200 \mu \mathrm{m}$ ). Smaller distances between the continuous phase channel and the orifice lead to larger strain rates acting on the dispersed phase for the same continuous phase flow rate [130], therefore leading to the thinner streams of dispersed phase required for dripping and jetting breakup modes at low Ca.

Within the geometry controlled breakup mode, increasing the flow rate ratio results in the droplet diameter approaching a limit that is close to the size of the orifice: the droplet size did not appear to change significantly for $12.5<\varphi<20$. Only once the droplets are formed via the dripping mode do they become smaller in diameter than the orifice, and still they are relatively large in the context of conventional emulsions. Indeed, due to their size it was not possible to keep the droplets dispersed. Even when materials with densities close to $1000 \mathrm{~kg} \mathrm{~m}^{-3}$ were used as the dispersed phase, the droplets would not remain dispersed for longer than several seconds. The minimum droplet size is primarily controlled by the orifice size. It was not possible to form droplets significantly smaller than those shown in figure 5.2 (f), because as the flow rate ratio was further increased, instabilities in the droplet formation process occurred, 
leading to larger, polydisperse droplets. To obtain a wide range of droplet sizes, a variety of microfluidic devices needs to be used. As a consequence of the creaming, it was not possible to control the volume fraction of the emulsions.

\subsubsection{Emulsion purification}

As discussed in chapter 2, unless droplets are formed in the jetting mode, satellite droplets are also produced. It is possible to passively remove these droplets immediately following their formation by designing a microfluidic device that sorts droplets based on their size $[131,132]$. However, lacking the fabrication facilities to produce such a microfluidic device, the crude emulsions were filtered using a homemade centrifuge filter to separate the main droplets from their satellites.

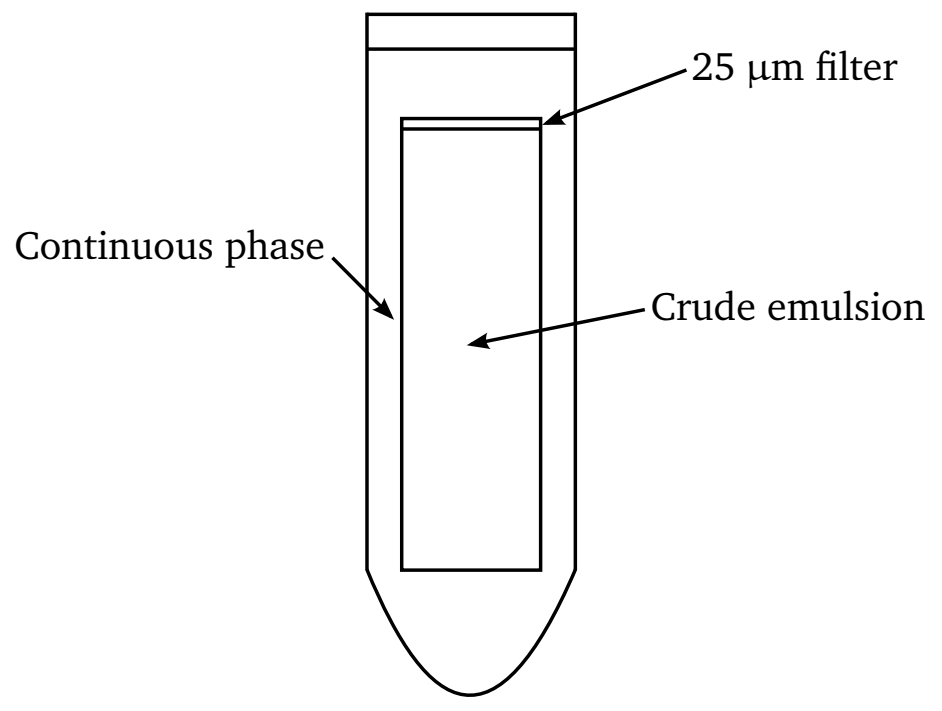

Figure 5.4: A schematic depiction of the centrifuge filter used for emulsion purification.

A depiction of the centrifuge filter is given in figure 5.4. The crude emulsion is held beneath a filter with pore sizes of approximately $25 \mu \mathrm{m}$ within a $30 \mathrm{~cm}^{3}$ sample tube that is encased in a $50 \mathrm{~cm}^{3}$ centrifuge tube filled with continuous phase. Upon centrifugation, the satellite droplets are able to pass through the filter, whereas the larger droplets are not. The satellite droplets can then be decanted from the centrifuge tube leaving a truly monodisperse emulsion behind. In figure 5.5 is a purified emulsion after 30 minutes of centrifugation at $100 \mathrm{rpm}$ and the isolated satellite droplets.

Consideration was made to using the isolated satellite droplets as a method to obtain droplets smaller than could be obtained directly using our microfluidic device. Unfortunately, investigation of the isolated satellite droplets showed that they were highly polydisperse. The polydispersity appeared to be independent of the flow rate ratio and the dispersed phase viscosity, as polydimethylsiloxane test emulsions also formed with highly polydisperse satellites.

\subsubsection{Polydisperse emulsions}

One of the goals of this research programme was to increase our understanding of how polydispersity influences emulsion rheology. A first approximation of polydispersity can be created by forming bimodal systems, which can be created easily by mixing two purified monodisperse emulsions in varying proportions. Emulsions A and D were mixed to give samples with specific number fractions $x_{n}$ of the two differently 


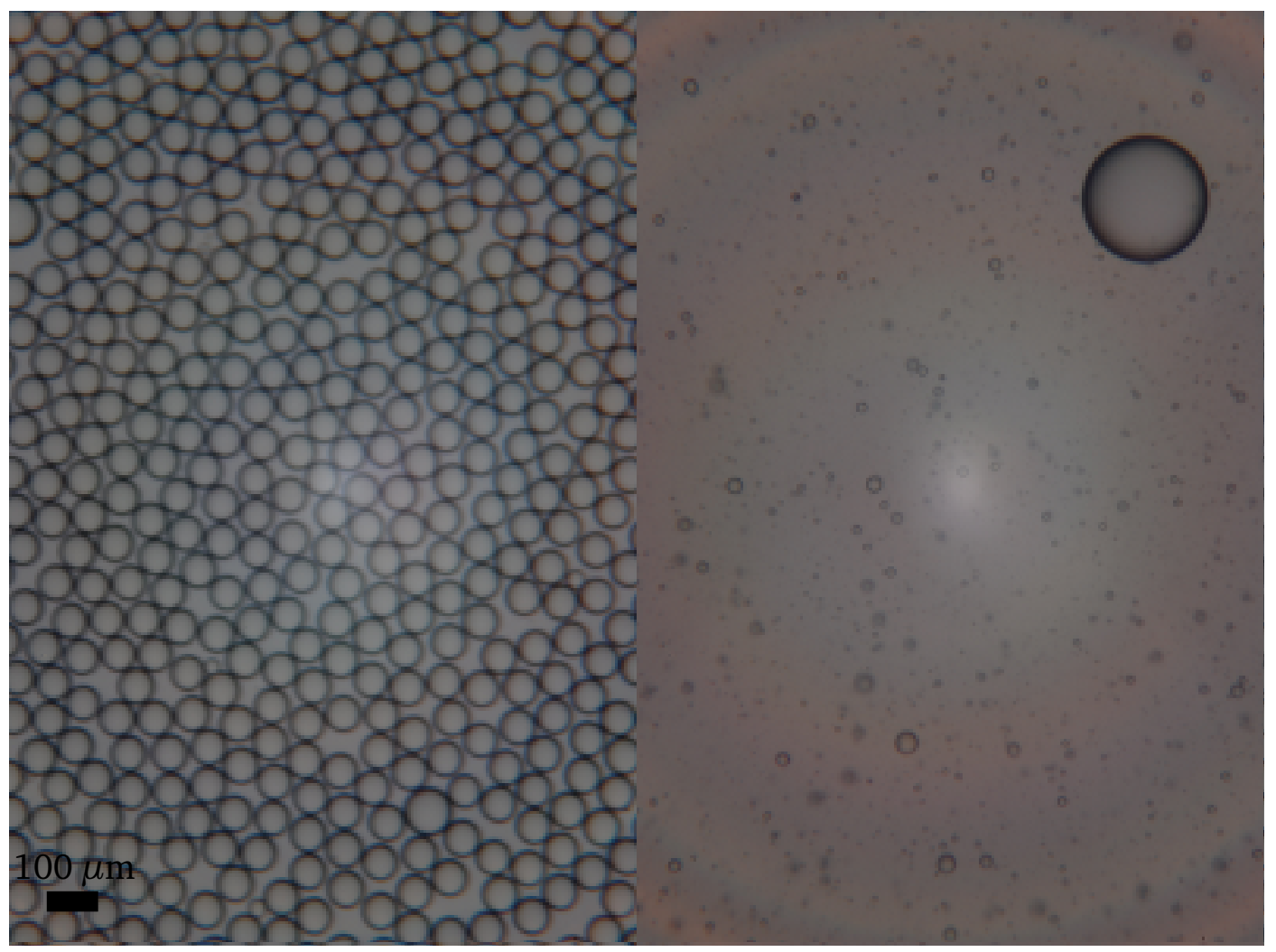

Figure 5.5: A purified emulsion (left) and the isolated satellite droplets (right).

sized droplets ranging from $100 \%$ of emulsion A to $100 \%$ of emulsion D. The bimodal emulsions and their exact composition are summarised in table 5.2.

Table 5.2: The bimodal emulsions.

\begin{tabular}{ccc}
\hline \multicolumn{3}{c}{ Table 5.2: The bimodal emulsions. } \\
\hline Sample name & Volume fraction of A & Volume fraction of D \\
\hline PD1 & 0.9 & 0.1 \\
PD2 & 0.75 & 0.25 \\
PD3 & 0.5 & 0.5 \\
PD4 & 0.25 & 0.75 \\
PD5 & 0.1 & 0.9 \\
\hline
\end{tabular}

These number fractions made it possible to establish how the structure of the droplets is altered and the associated rheological properties of emulsions that range from systems of large droplets perturbed by smaller droplets to the opposite extreme.

\subsection{Emulsion characterisation}

\subsubsection{Optical microscopy}

\subsubsection{Experimental details}

A Nikon OPTIPHOT2-POL optical microscope was used to image the emulsions during formation and after collection. Qualitative estimates of droplet size could be obtained using the internal graticule that was calibrated against a standard, while more accurate 
measurements were made using JEOL Semaphore imaging software. In this case, the size of the droplets were calculated relative to a section of the image of known size, which could be any one of the channels within the microfluidic device also captured in the micrograph. Images were taken for analysis using a Nikon D200 digital single-lens reflex camera attached to the microscope using either 4 or 10 times magnification. The captured images were of sufficient resolution ( 3872 by 2592 pixels) to ensure that the interface of the droplets was composed of several pixels. Because of this, there was little chance of errors arising when defining the diameter of a droplet when using Semaphore.

\subsubsection{Results}

After the analysis of 30 droplets from each filtered sample, the results shown in table 5.1 were obtained. These results confirm the qualitative observations made in section 5.1.2, where higher flow rate ratios create emulsions with smaller droplets. All emulsions showed a low degree of variance in their droplet size.

\subsubsection{Nuclear magnetic resonance}

\subsubsection{Experimental details}

All NMR measurements were made using a Bruker AVANCE 400 spectrometer with a field strength of $9.4 \mathrm{~T}$, which correlates to a $400 \mathrm{MHz}{ }^{1} \mathrm{H}$ Larmor precession frequency. The magnetic field gradients were generated using a Bruker Diff 60 probe. Samples that had been left to stand overnight of the emulsion cream were placed in $5 \mathrm{~mm}$ Norell XR-55-7 glass NMR tubes to a depth of approximately $15 \mathrm{~mm}$.

Each stimulated echo experiment was performed at $295 \mathrm{~K}$ and with a maximum gradient strength ranging from 0.51 to $1.5 \mathrm{~T} \mathrm{~m}^{-1}$ (sample dependent to avoid excessive attenuation in larger emulsion droplets) in 128 discrete steps with a 1 ms gradient duration. The gradient duration was restricted to ensure that the following relation holds

$$
\delta \ll \frac{a^{2}}{D}
$$

which means that a minimal number of molecules experience a droplet boundary during the gradient pulse and the SGP approximation holds [56]. The range of gradient strengths is suitable for measuring confining geometries with characteristic distances as small as $23 \mu \mathrm{m}$, and was set specifically for each sample based on the droplet size estimated from optical microscopy. Experimental data was collected using TopSpin 2.0 and processed using a combination of the Curve Fitting Toolbox for Matlab R2009a and Prospa 2.2.15.

\subsubsection{Results and discussion}

The ${ }^{1} \mathrm{H}$ spectrum for all emulsions contains two peaks: one at $1 \mathrm{ppm}$ corresponding to dodecane and the other at $3 \mathrm{ppm}$ attributed to water. Because Pluronic F127 was present at a relatively low concentration, no resonance peaks associated with the surfactant were evident. By integrating the peaks it was possible to determine the volume fraction of the emulsions using the following relationship [133]

$$
\frac{V_{d}}{V_{c}}=\frac{I_{d}\left[m\left(H_{d}\right) \rho_{d}\right]^{-1}}{I_{c}\left[m\left(H_{d}\right) \rho_{c}\right]^{-1}},
$$




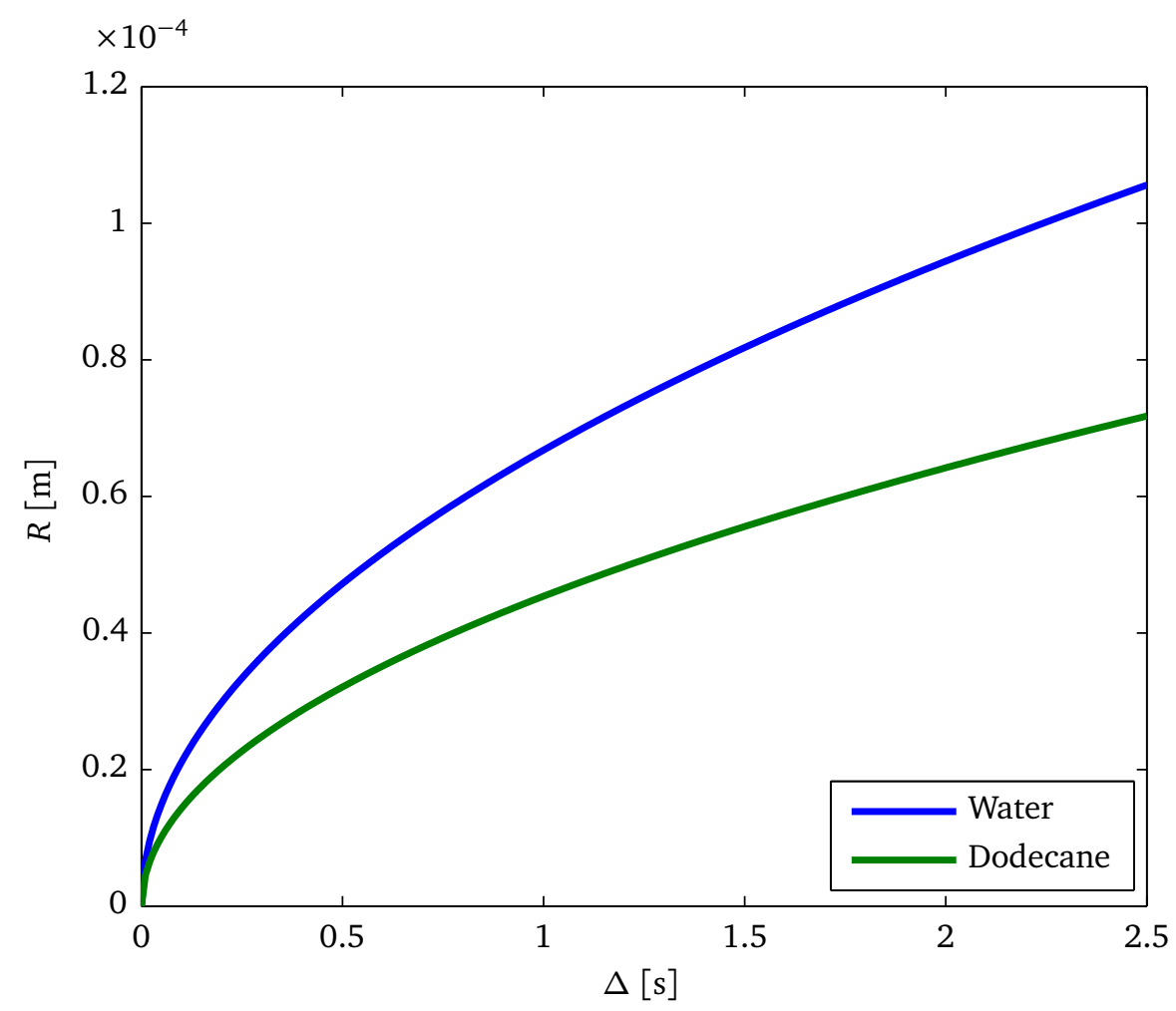

Figure 5.6: The displacement of water and dodecane molecules, as predicted by equation 3.8.

where $I$ is the magnitude of the integral, $m(H)$ is the mass fraction of protons and $\rho$ is the bulk density. Subscripts indicate the relevant phase. For all monodisperse emulsions, equation 5.3 gave $\phi \approx 0.74$, indicating the emulsions are close packed.

The separation between the two resonance peaks for dodecane and water meant that data for the diffusion of the continuous and dispersed phases could be collected simultaneously but analysed independently. Additionally, because of the large droplet size, the motion of the droplets themselves could be ignored during analysis.

Dispersed phase diffusion A STE pulse sequence and an inversion-recovery test were used to measure the free diffusion coefficient for dodecane and its $T_{1}$ relaxation time as $1.03 \times 10^{-9} \mathrm{~m}^{2} \mathrm{~s}^{-1}$ and $1.26 \mathrm{~s}$, respectively. Both measurements are in agreement with standards in the literature [134] and were used to establish parameters for measuring the diffusion of dodecane in the emulsions so that a sufficient number of molecules would experience restricted diffusion. In figure 5.6 the expected displacement of dodecane molecules at observation times up to $2.5 \mathrm{~s}$ is shown.

Using emulsion E with $a=29 \mu \mathrm{m}$ as an example and the information summarised in figure 5.6, we predict that a significant amount of the diffusing molecules will come in contact with the boundary when $\Delta \geq 500 \mathrm{~ms}$. When the diffusion of dodecane in emulsion $\mathrm{E}$ was measured at varying $\Delta$ and plotted as a function of $q$, a coherence feature was observed to form when $\Delta=500 \mathrm{~ms}$ at $q \approx 24000 \mathrm{~m}^{-1}$ that becomes more defined as $\Delta$ is further increased and a larger number of molecules come in contact with the boundary, as shown in figure 5.7.

As discussed in section 3.2.3.2, this minima corresponds to a characteristic distance in the sample, and occurs when the numerator of equation 3.27 is equal to zero. In other 


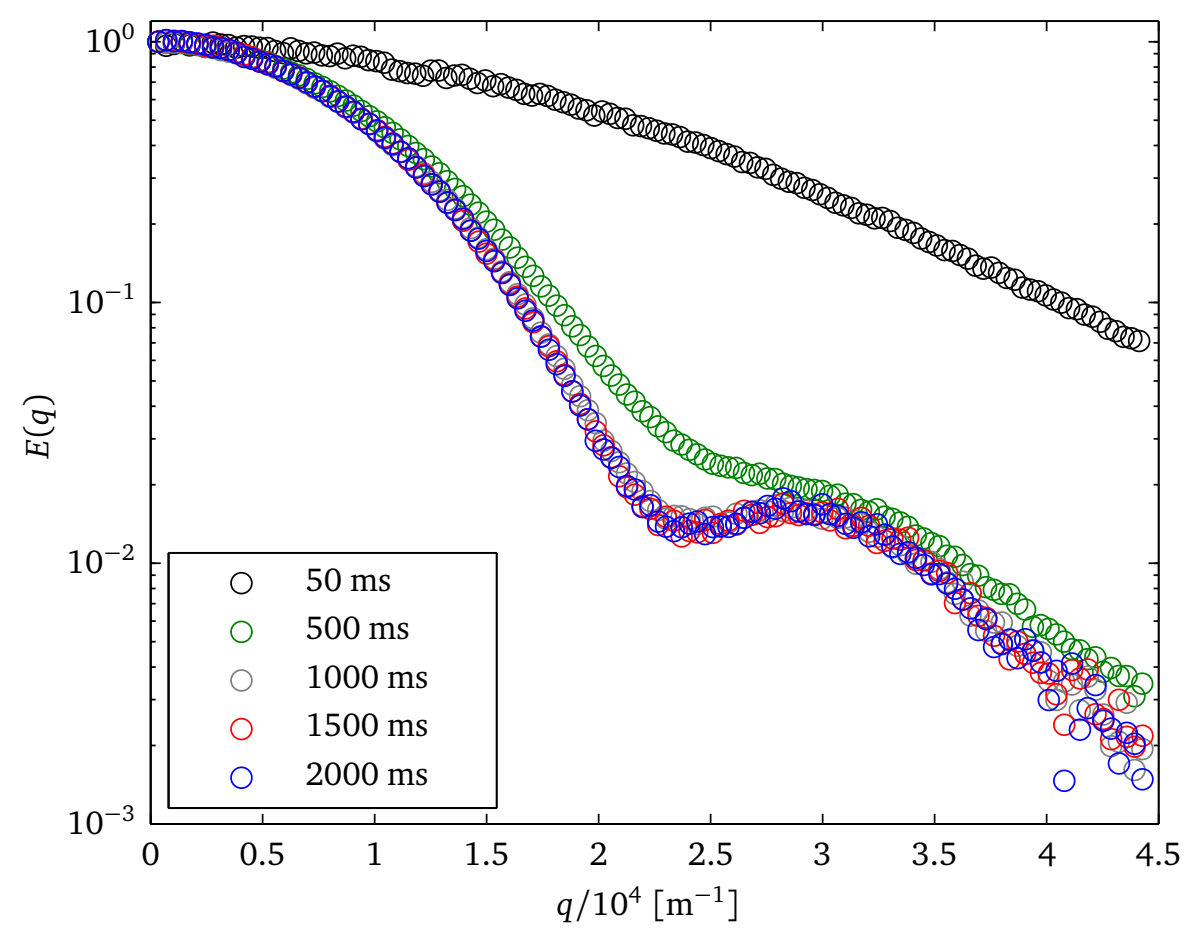

Figure 5.7: A comparison of the signal attenuation for dodecane in emulsion $\mathrm{E}$ at various $\Delta$. At $\Delta=50 \mathrm{~ms}$, there is little restricted diffusion. At $\Delta=500 \mathrm{~ms}$, the effects of restricted diffusion can be seen, with a coherence feature formed at approximately $2.5 \times 10^{4} \mathrm{~m}^{-1}$. When $\Delta \geq 1000 \mathrm{~ms}$, the coherence feature becomes well defined and the signal attenuation is nearly identical for higher $\Delta$.

words, when

$$
(2 \pi q a) \cos (2 \pi q a)=\sin (2 \pi q a) .
$$

The positions of the minima can be determined using the following relation

$$
2 \pi q a=\frac{\sin (2 \pi q a)}{\cos (2 \pi q a)}
$$

which leads to the simple result of

$$
a=\frac{0.715}{q}
$$

Using equation 5.5 for emulsion E gives $a=30 \mu \mathrm{m}$, which is concordant with the measurement made using optical microscopy (table 5.1).

The efficacy of measuring the positions of the coherence minima is reduced as the droplets increase in size, ensuring that, while a majority of the molecules may be experiencing restricted diffusion, a sufficiently large proportion are not, resulting in the minima being poorly defined. This is shown in figure 5.8 , where it is difficult to determine the position of the minimum in the $q$-space plot for emulsion A when $\Delta=2500 \mathrm{~ms}$.

When Woodward et al. [76] encountered a similar issue, they suggested the use of equation 3.31, which is repeated in full below: 


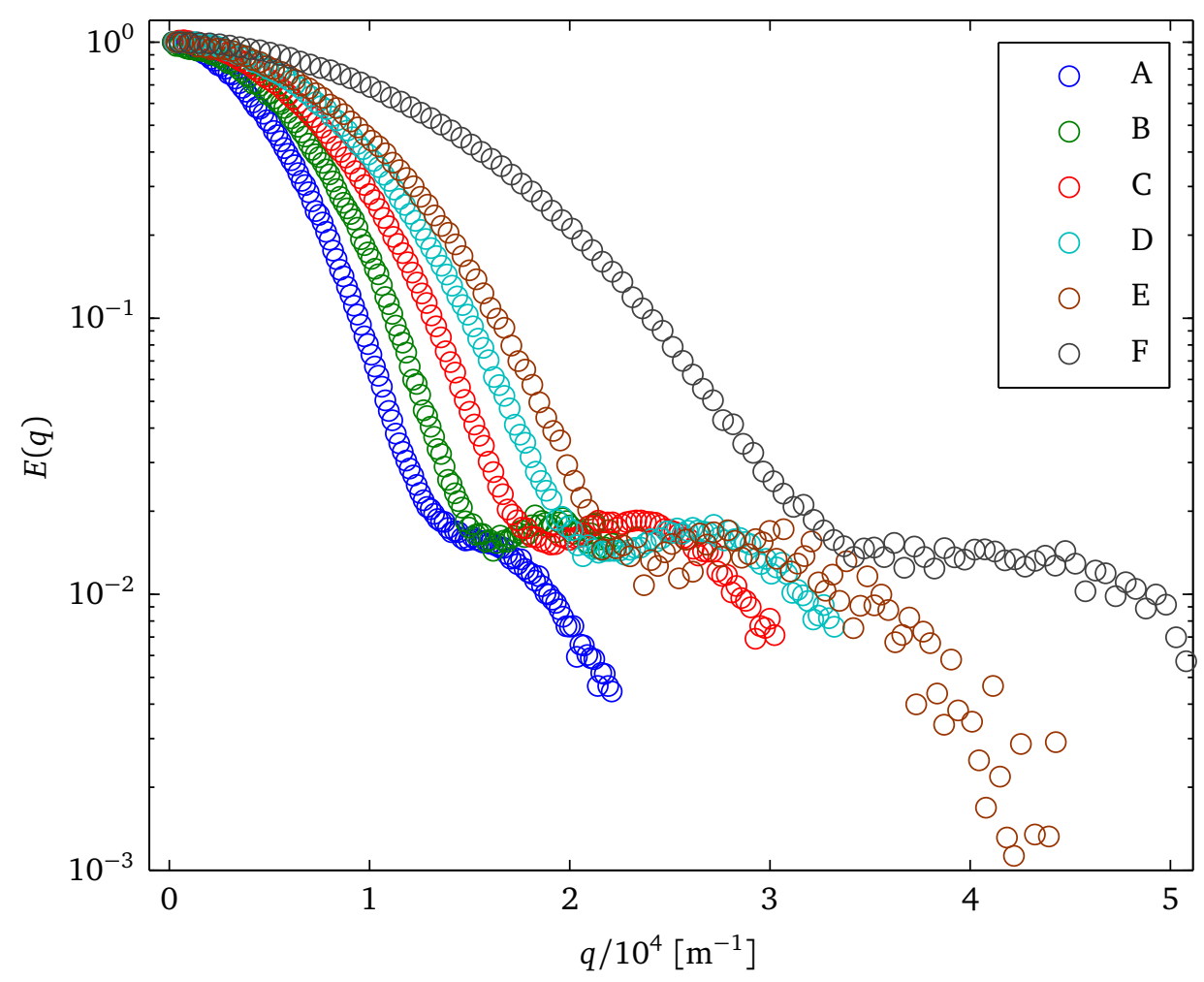

Figure 5.8: A demonstration of how the coherence feature is affected by the proportion of molecules experiencing restricted diffusion. Measurements were made at $\Delta=2500 \mathrm{~ms}$ for emulsions A-E, and $\Delta=1500 \mathrm{~ms}$ for emulsion $\mathrm{F}$.

$$
\begin{array}{r}
E(q, \Delta)=x\left(\frac{9[(2 \pi q a) \cos (2 \pi q a)-\sin (2 \pi q a)]^{2}}{(2 \pi q a)^{6}}\right)+ \\
(1-x) \exp \left(-(2 \pi q)^{2} D \Delta\right) .
\end{array}
$$

The first part of the equation represents the contribution from the molecules experiencing restricted diffusion, and the second part expresses the contribution from the molecules undergoing free diffusion. The weighting factor $x$ gives the proportion of molecules that come in contact with the boundary.

A fit to experimental data obtained for emulsion $\mathrm{E}$ with $\Delta=2500 \mathrm{~ms}$ using equation 5.6 is shown in figure 5.9. It is not concordant with the plots presented by Woodward et al. in [76] for an emulsion with $a=13.5 \mu \mathrm{m}$ and $D=1.09 \times 10^{-10} \mathrm{~m}^{2} \mathrm{~s}^{-1}$ shown in figure 3.6. One difficulty in applying equation 5.6 to diffusion data is that it is not clear in the assigned literature $[76,133]$ whether $D$ is taken as a fixed or free parameter. Both approaches have been used here. In the first, $D$ was set to $1.03 \times 10^{-9} \mathrm{~m}^{2} \mathrm{~s}^{-1}$, the bulk self-diffusion coefficient of dodecane. This would be the fastest applicable diffusion coefficient for the molecules undergoing unrestricted diffusion. As a result of this relatively fast diffusion coefficient, $\exp \left(-(2 \pi q)^{2} D \Delta\right) \rightarrow 0$ at small $q$ values, this thereby ensures the presence of sharp minima in the fit - there is no contribution from the second term of the equation at $q$-values associated with the length scale of the geometry (figure 5.9).

If instead $D$ is treated as a free fitting parameter, the result shown in figure 5.10 is 
obtained. Here $D=1.03 \times 10^{-11} \mathrm{~m}^{2} \mathrm{~s}^{-1}$. The determined fit is a better representation of the data, however the diffusion coefficient is significantly lower than expected to accurately represent the contribution from free diffusion to the signal attenuation. Our data has highlighted the limitations of the weighted average of equation 5.6, which appears to be valid only for molecules with diffusion coefficients in the order of $10^{11} \mathrm{~m}^{2} \mathrm{~s}^{-1}$ and slower. Further developments of the theory may include a multiplicative relationship or higher order terms to account for the shallow minima.

Despite the visual discrepancy between the two approaches and the highlighted weaknesses, both produce primary minima that are consistent with droplet sizes obtained from optical microscopy and are, at most, $2 \mu \mathrm{m}$ apart from each other. The results are summarised in table 5.3.

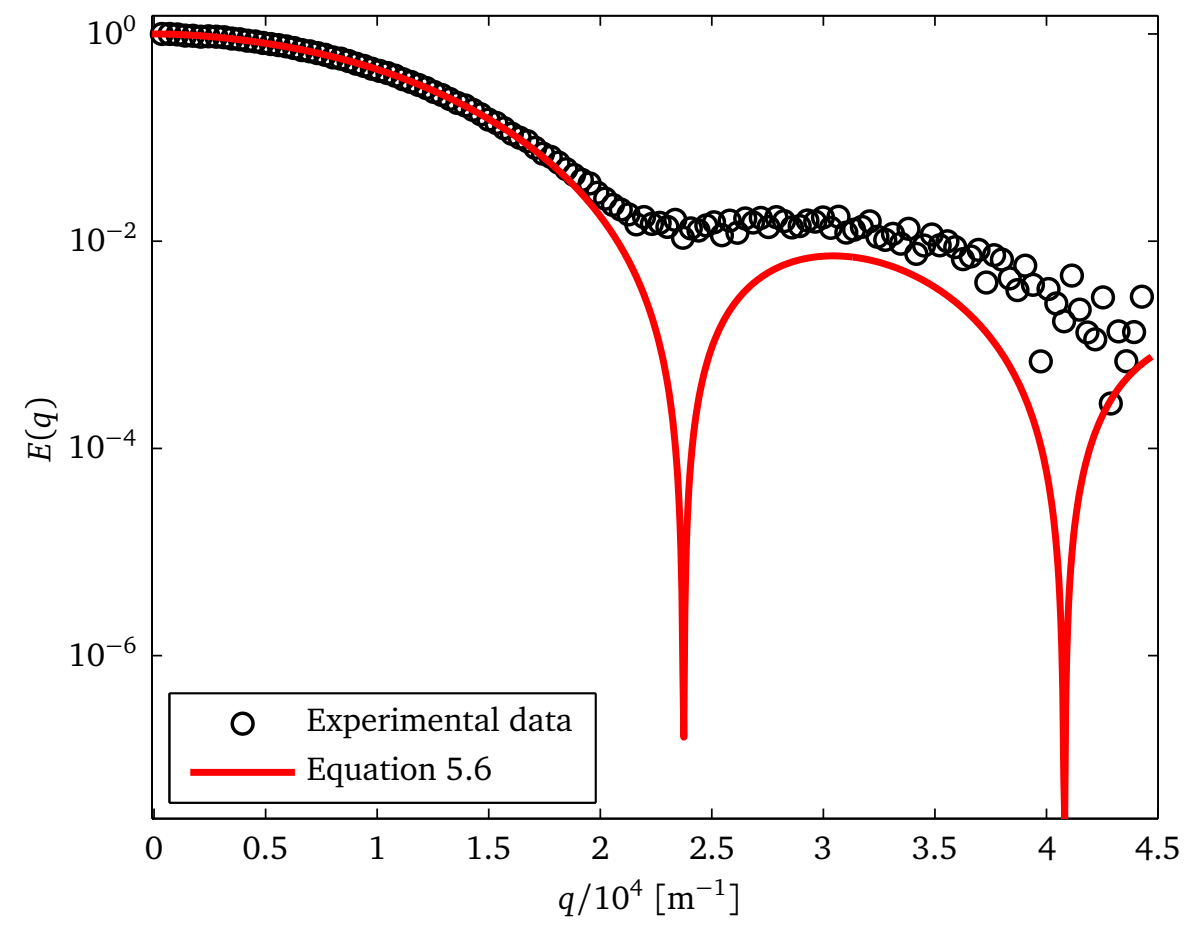

Figure 5.9: A plot showing the discrepancy between experimental data for emulsion $\mathrm{E}$ with $\Delta=2500 \mathrm{~ms}$ and the attenuation predicted by equation 5.6.

Table 5.3: Emulsion droplet radii as obtained with dispersed phase diffusive diffraction.

\begin{tabular}{ccc}
\hline Emulsion & $\boldsymbol{a}_{\text {NMR }}[\boldsymbol{\mu m}]$ & Weighting factor \\
\hline A & $50.9 \pm 0.5$ & 0.8443 \\
B & $43.4 \pm 0.3$ & 0.9318 \\
C & $37.7 \pm 0.3$ & 0.9817 \\
D & $33.3 \pm 0.1$ & 0.9926 \\
E & $30.1 \pm 0.2$ & 0.9942 \\
F & $19.6 \pm 0.2$ & 0.9989 \\
\hline
\end{tabular}

Restricted diffusion of the dispered phase in the bimodal samples will give us the proportion of each constituent emulsion (which is already known), but no information 


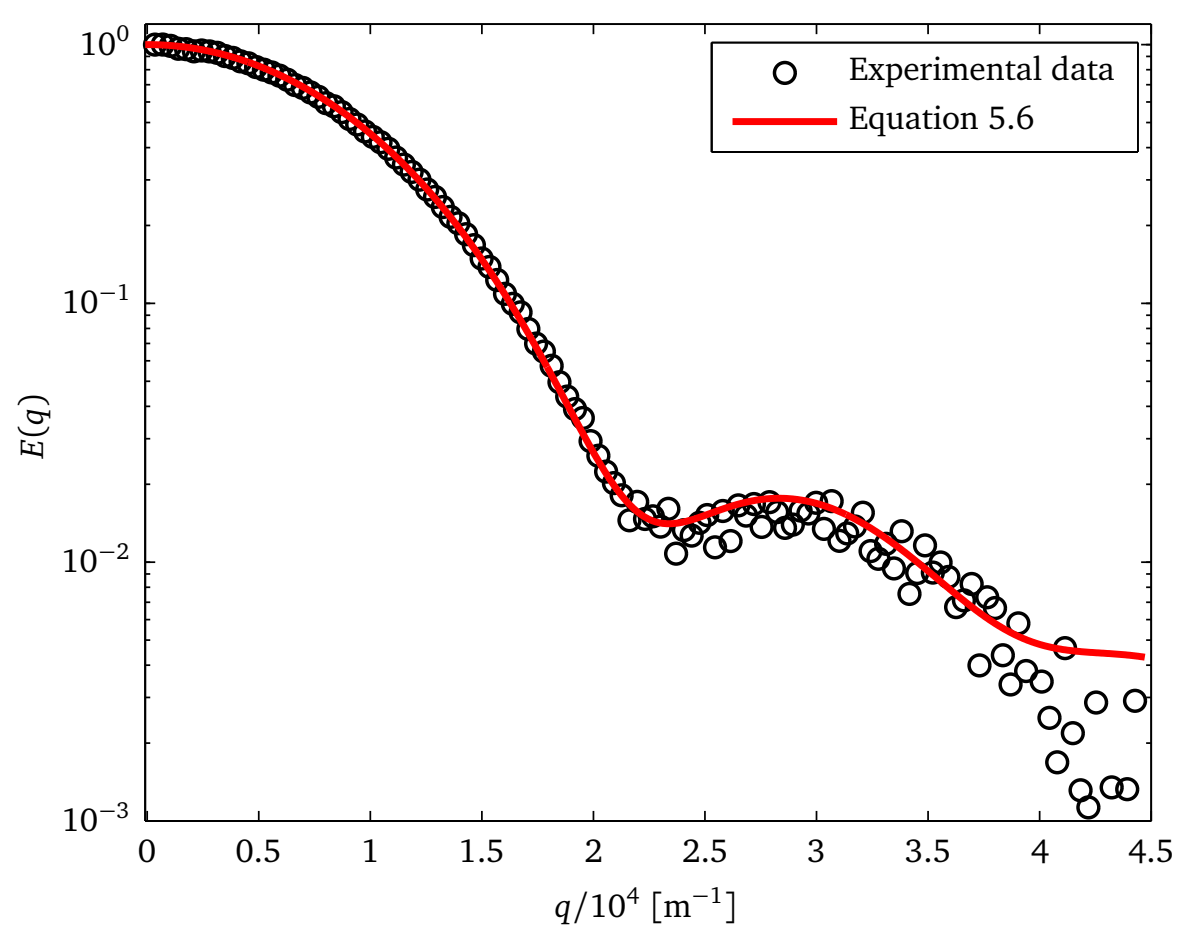

Figure 5.10: A plot showing the better fit obtained for duffusion of dodecane in emulsion $\mathrm{E}$ with $\Delta=2500 \mathrm{~ms}$ if $D$ is left as a free fitting parameter.

about how the droplets are dispersed through the sample can be obtained. For this we must measure the diffusion of the continuous phase.

Continuous phase diffusion Continuous phase molecules also produce coherence features as a result of direct contact with the interface of an emulsion droplet. While it is possible to obtain the droplet size from such data, continuous phase diffusive diffraction also provides information about the structure of the emulsion [65], such as the type and order of the packing. Using the pore hopping theory developed by Callaghan et al. [135], it is possible to relate the signal attenuation to the size of the pores $o$, the distance between the pores $b(b=2 a)$, and the standard deviation in the pore size $\xi$. The fiting expression is [135]

$$
E(q, \Delta)=\left|S_{0}(q)\right|^{2} \exp \left(-\frac{6 D_{\mathrm{eff}} \Delta}{b^{2}+3 \xi}\left[1-\exp \left(-2 \pi^{2} q^{2} \xi^{2}\right) \frac{\sin (2 \pi q b)}{2 \pi q b}\right]\right),
$$

where the form factor for a spherical matrix such as a close packed emulsion is given by

$$
\left|S_{0}(q)\right|^{2}=\frac{9[(2 \pi q a) \cos (2 \pi q a)-\sin (2 \pi q a)]^{2}}{(2 \pi q a)^{6}} \text {. }
$$

A fit of equation 5.7 to experimental data from emulsion $\mathrm{C}$ with $\Delta=1000 \mathrm{~ms}$ is shown in figure 5.11. While the first minimum is well modelled, the second minimum is not predicted. This has previously been observed when characterising monodisperse emulsions with radii from 12 to $20 \mu \mathrm{m}$ [136], and is attributed to the model not accounting for diffusion of the molecules beyond the first shell of pores $[135,136]$. 
Despite this, the results shown in table 5.4 for the droplet diameter are concordant with droplet radii obtained from diffusive diffraction of the dispersed phase and optical microscopy.

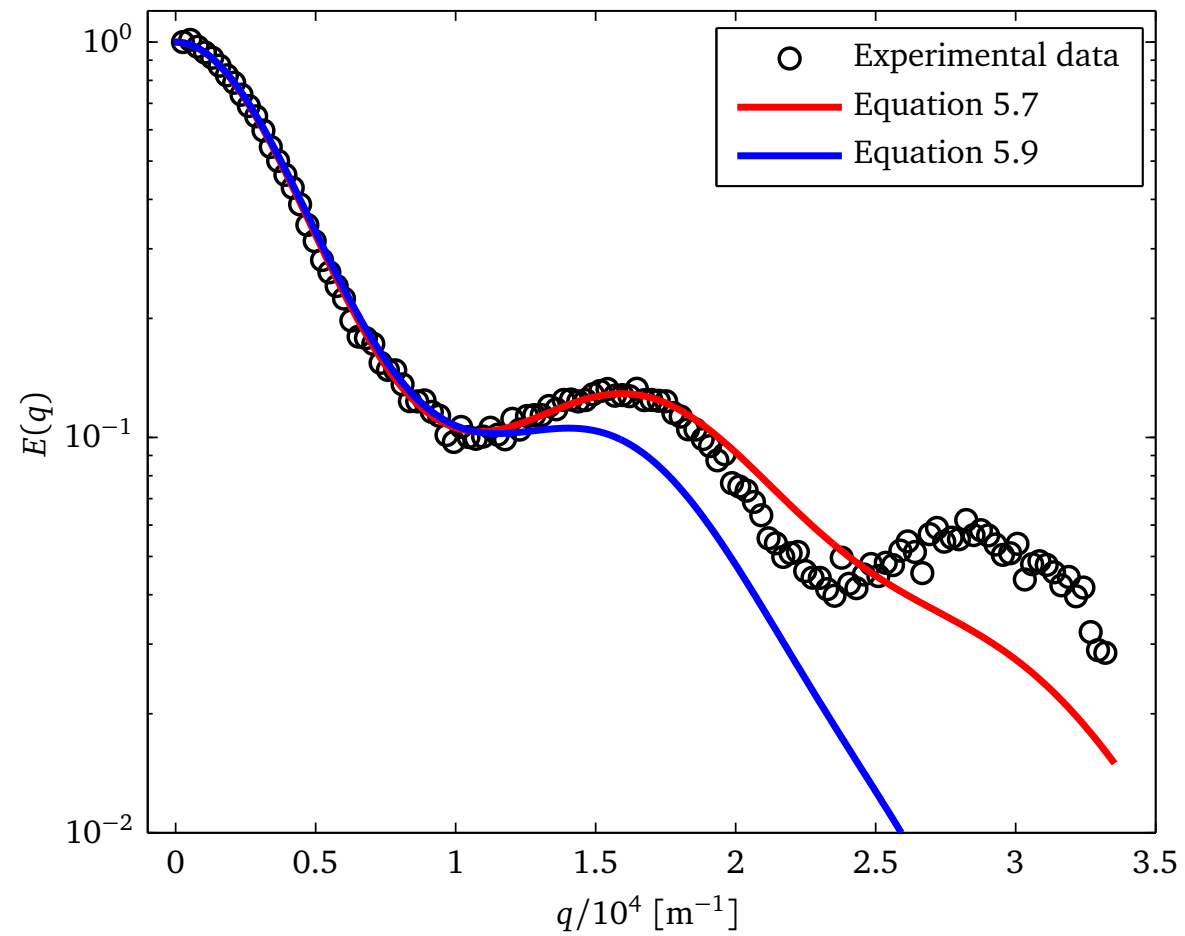

Figure 5.11: A fit of the continuous phase diffusion data for emulsion $\mathrm{C}$ obtained using equations 5.7 and 5.9 where $\Delta=1000 \mathrm{~ms}$ and $\delta=1 \mathrm{~ms}$.

Table 5.4: Parameters obtained from pore hopping theory.

\begin{tabular}{ccc}
\hline Emulsion & $\boldsymbol{o}[\mu \mathrm{m}]$ & $\boldsymbol{b}[\mu \mathrm{m}]$ \\
\hline A & $27 \pm 3$ & $100 \pm 10$ \\
B & $25 \pm 1$ & $88 \pm 3$ \\
C & $23 \pm 1$ & $74 \pm 2$ \\
D & $15.4 \pm 0.5$ & $70 \pm 1$ \\
E & $14 \pm 1$ & $59 \pm 3$ \\
F & $10 \pm 1$ & $41 \pm 1$ \\
\hline
\end{tabular}

Because the lattice of droplets is regularly spaced with nearly perfect hexagonal packing, fitting equation 5.7 to experimental data produced physically impossible values of $\xi$, and the values of the pore size standard deviation have not been reported. The pore hopping expression for a lattice of perfectly monodisperse spheres is [137]

$$
E(q, \Delta)=\left|S_{0}(q)\right|^{2} \exp \left(-\frac{6 D_{\mathrm{eff}} \Delta}{b^{2}}\left[1-\frac{\sin (2 \pi q b)}{2 \pi q b}\right]\right),
$$

where $\left|S_{0}(q)\right|^{2}$ is equal to equation 5.8 , and provides a fit that is similar to equation 
5.7 until the first minimum (figure 5.11). The deviation at high $q$-values is likely to be the result of "effective polydispersity" induced by the dynamics of the oil-water interface which is not accounted for in the model that is based on spherical glass beads with rigid surfaces. Small rearrangements of the interface due to the thermal energy of the system would effectively change the pore shape and size, as well as creating alternative pathways for diffusion. It is possible that the presence of an extra free parameter in equation 5.7 in comparison to equation 5.9 better accounts for this deviation from ideal behaviour. In addition, inaccuracy is induced by diffusion of water molecules beyond the first shell of pores, as mentioned previously.

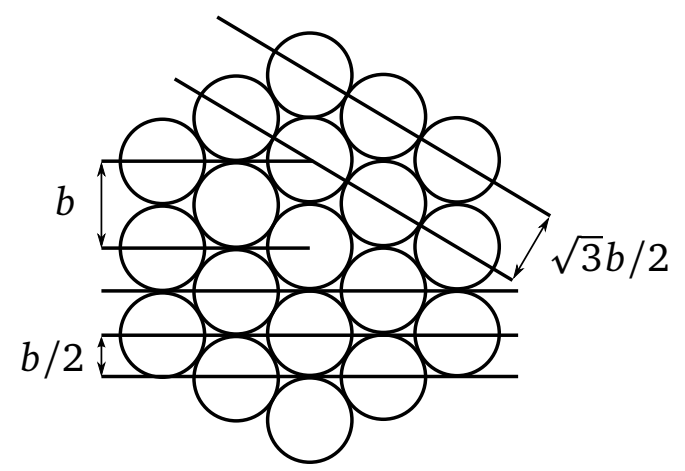

Figure 5.12: A depiction of the characteristic distances in an array of hexagonally close packed spheres. Adapted from [138].

Håakansson, Pons and Söderman [138] identified that the maxima and minima observed in diffusive diffraction of the continuous phase correlated to diffusion between pores in a hexagonal lattice of different length scales, as shown in figure 5.12.

The first and second maxima correspond to diffusion lengths of $\sqrt{3} b / 2$ and $1 / 2 b$, respectively, while the first minimum corresponds to a diffusion length of $b$ [138]. If there is a degree of hexagonal close packing of the droplets, there will be maxima at $1 / 2 q$ and $\sqrt{3} q / 2$ and a minimum at $q$ corresponding to one, or both, of the constituent emulsions. Disruption of the hexagonal packing will see these coherence features become diminished or not appear at all.

The diffusive diffraction of the continuous phase in the bimodal samples is shown in figures 5.13 and 5.14. As expected, there is significant dampening of the coherence features. Despite this, it was possible to gain a qualitative understanding of the disorder in the structures by considering the coherence features present for the constituent monodisperse emulsions.

In figure 5.13 is shown the bimodal samples that could be considered as a matrix of large droplets perturbed by smaller droplets, as well as the constituent emulsions and a 50:50 mix for comparison. It is important to bear in mind that NMR is a volume based technique. In other words, a large number of small droplets may produce a signal of equal magnitude to that of one larger droplet if the disparity in the radii is significant. The samples PD1 and PD2 generate similar signal attenuation to that of emulsion A, suggesting that a significant proportion of the sample is made up of domains of hexagonally packed larger droplets. That is, at moderate to low concentrations, the smaller droplets are dispersed through the sample in a way that has minimal effect on the original structure.

The sample with equal numbers of droplets of each size, PD3, shows similar signal 


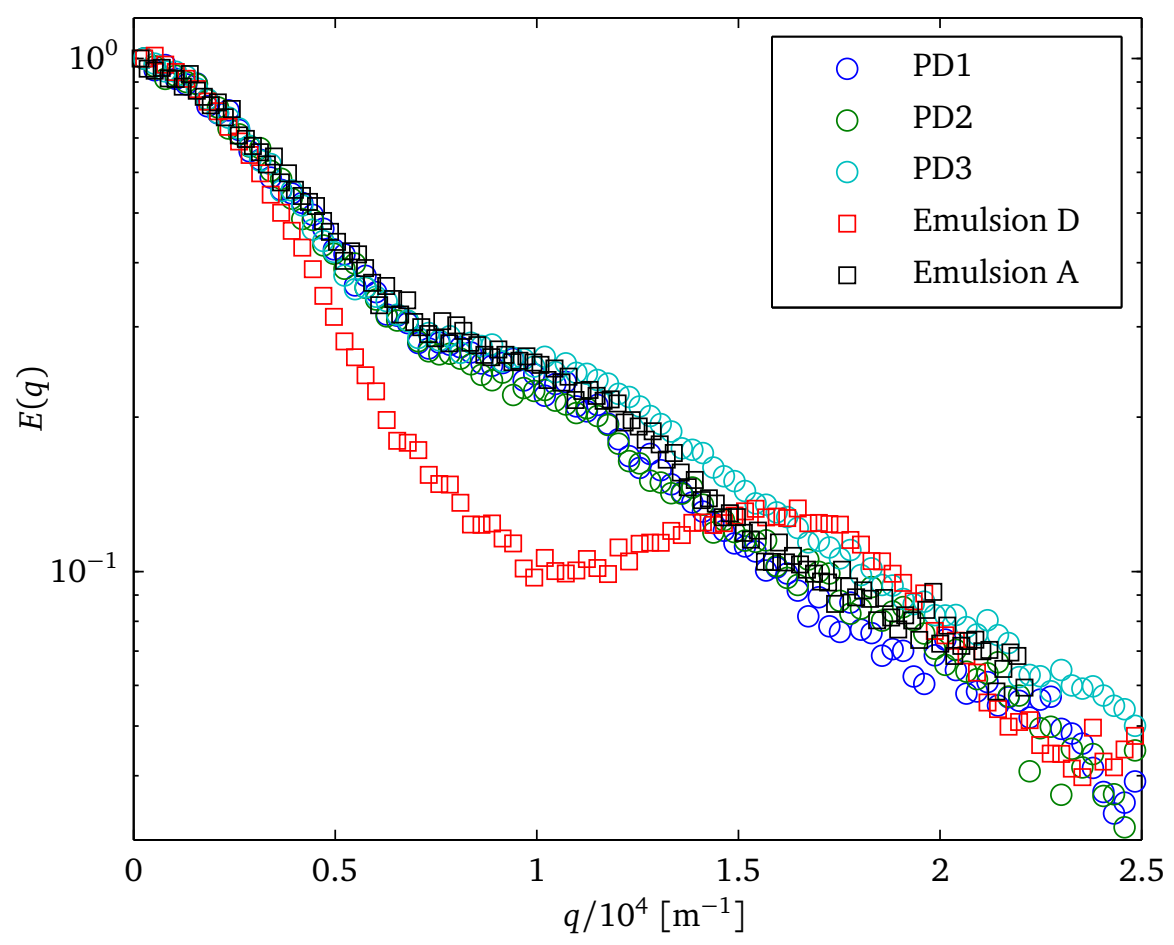

Figure 5.13: A comparison of some bimodal emulsions (circles) with their constituent monodisperse emulsion (squares) at $\Delta=1000 \mathrm{~ms}$. The overall structure of these bimodal samples is similar to that of a monodisperse emulsion.

attenuation to emulsion A at lower $q$ values, but deviates slightly at higher $q$. The deviation does not coincide with an expected maxima at $q \approx 17500 \mathrm{~m}^{-1}$ that would indicated packing of the smaller droplets.

In figure 5.14 is shown the bimodal samples with a higher number fraction of small droplets, as well as the comparison cases. The dampened minimum at $q \approx 10000 \mathrm{~m}^{-1}$ in the signal attenuation for PD4 suggests that there is a similar amount of domains of hexagonally packed large and small droplets through the sample. Somewhat counterintuitively, as the number fraction of larger droplets is decreased, the signal attenuation resembles neither constituent emulsion. The perturbation of large droplets on a matrix of smaller ones is demonstrated by the micrographs in figure 5.15.

It is not possible to obtain physically meaningful quantitative data about the packing arrangement by fitting linear combinations of equation 5.7 that correspond to the proportions of each monodisperse emulsion in the sample. This is because the droplet packing within the bimodal sample is not simply a combination of the packings of each constituent monodisperse emulsion. An example fit is shown in figure 5.16.

Attempts were made to extract the distributions of characteristic length scales for the bimodal emulsions using a method developed by Kuchel, Eykyn and Regan [139] that involves applying a window function to the attenuation data, followed by taking the $n$th derivative and Fourier transformation. By taking the $n$th derivative prior to Fourier transformation, the coherence features are accentuated according to the following 


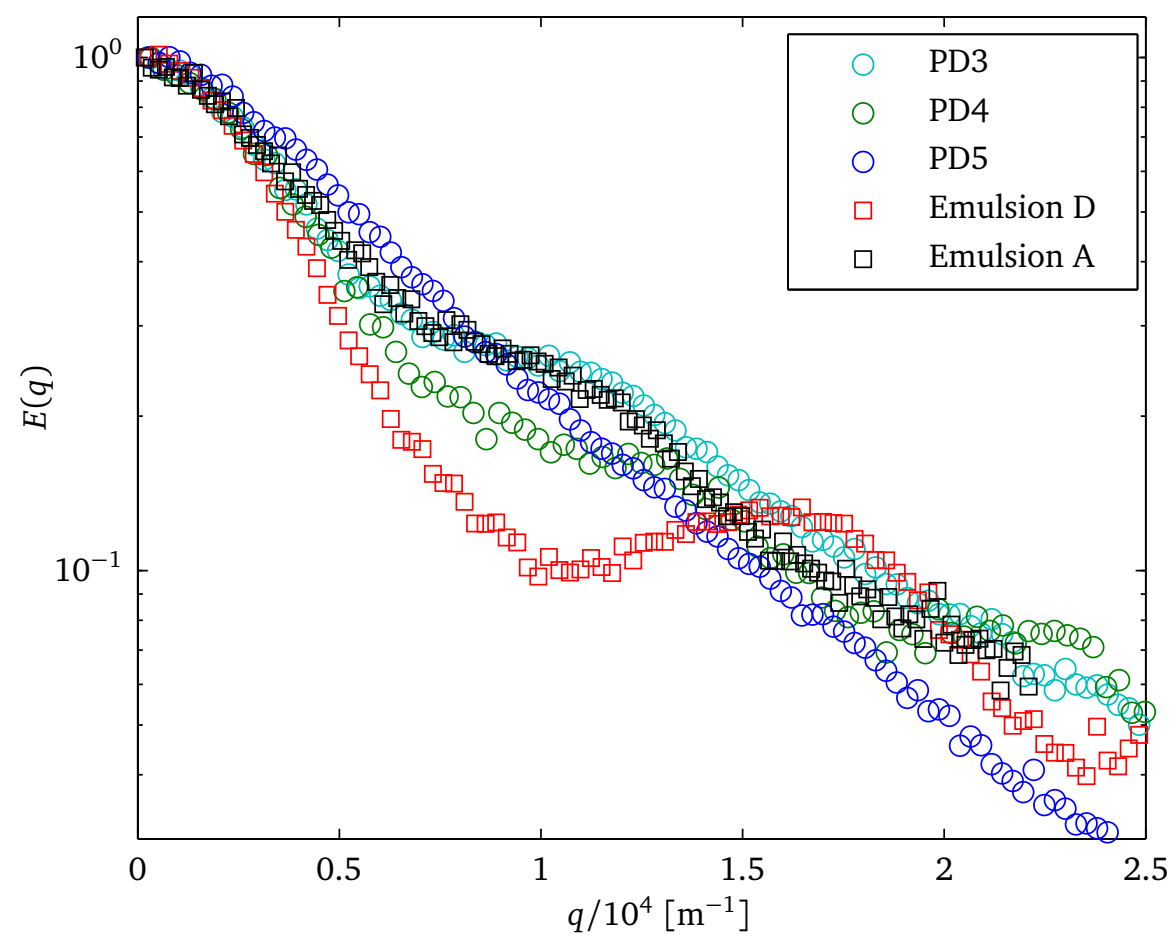

Figure 5.14: A comparison of different bimodal emulsions (circles) with their constituent monodisperse emulsion (squares) at $\Delta=1000 \mathrm{~ms}$. While the $0.75 \mathrm{~A}-0.25 \mathrm{D}$ emulsion shows some characteristics of the smaller droplets, the 0.9A-0.1D sample shows the behaviour of neither constituent monodisperse emulsion.
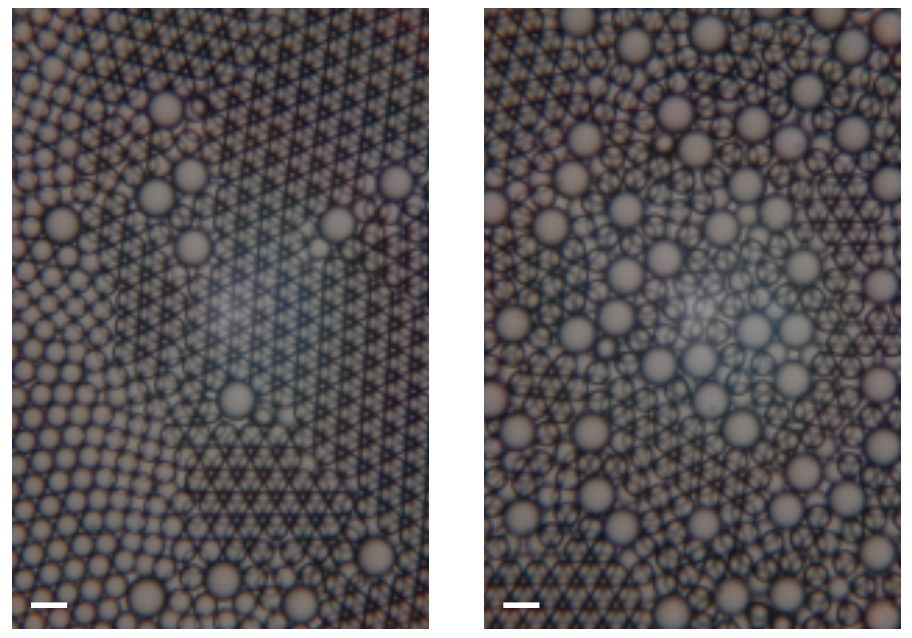

Figure 5.15: A depiction of the effect of increasing the concentration of large droplets dispersed through a matrix of smaller droplets.

relationship

$$
\frac{d^{n}}{d q^{n}} E(q, \Delta) \stackrel{\mathrm{FT}}{\rightarrow}(i 2 \pi q R)^{n} \overline{P_{s}}(R, \Delta)
$$

where characteristic distances appear as peaks when the mean propagator is plotted as a function of $q$ or displacement. The area of each peak gives the proportion of diffusing molecules that have experienced that respective characteristic distance. 


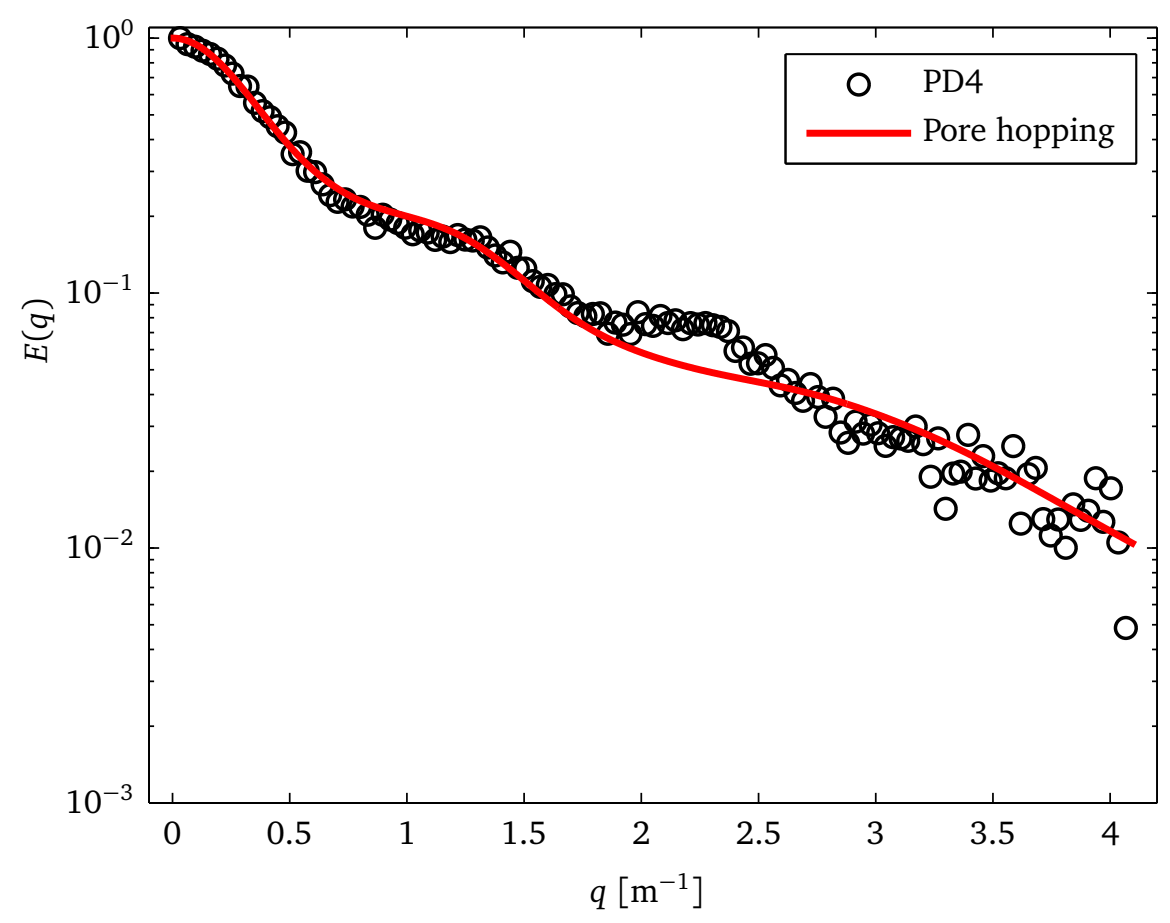

Figure 5.16: A fit of a linear combination of equation 5.7 to the attenuation data for PD4 with $\Delta=1000 \mathrm{~ms}$.

In figure 5.17 it is shown that this method is able to accentuate coherence in attenuation data from PD5 and clearly shows the presence of coherence features not associated with either constituent emulsion. When the data for the polydisperse emulsions are compared (figure 5.18) we see that each sample shares common coherence features, but that they are present in different proportions. It would be difficult to establish quantitative differences between the polydisperse droplet packings based on this data as it is not obvious to what type of packing arrangement each coherence feature corresponds. Additionally, it is possible that random errors in the $q$-space plots are being accentuated as much as, if not more, than legitimate coherence features. To determine quantitative information about the polydisperse systems, the experimental parameters $(\delta, \Delta$ and $g$ ) need to be further optimised for these systems.

The ${ }^{1} \mathrm{H}$ spectrum for each bimodal emulsion was used to determine the effect of the number fraction on droplet volume fraction using equation 5.3. For all emulsions, $\phi \approx 80$, as shown in table 5.5 .

Table 5.5: The volume fractions of the bimodal emulsions calculated using equation 5.3.

\begin{tabular}{cccccc}
\hline Emulsion & PD1 & PD2 & PD3 & PD4 & P5 \\
\hline Volume fraction & 0.79 & 0.81 & 0.78 & 0.80 & 0.80 \\
\hline
\end{tabular}

The increase in volume fraction from $\approx 0.74$ for the monodisperse emulsions is the result of the improved space filling efficiency that can be achieved by a polydisperse system [140]. For the ratio of droplet radii considered here $\left(a_{A} / a_{D}=1.67\right)$, the packing density does not change significantly with the number fraction. Only once the droplet radii ratio increases toward 10, where the smaller droplets are able to 
fit within interstitial spaces, does the number fraction significantly affect the volume fraction [141].

\subsection{Conclusion}

Six monodisperse emulsions were generated using a flow focusing microfluidic device and characterised using optical microscopy and PGSE NMR. By altering the flow rate ratio, emulsions with droplet radii from 20 to $50 \mu \mathrm{m}$ were obtained. At higher flow rate ratios, the droplet formation mode was observed to shift from geometry controlled to a dripping mechanism. After filtration of the emulsions formed by the geometry controlled mode to remove satellite droplets, all emulsions had little variance in their droplet radius.

Two of these monodisperse emulsions were used to create five bimodal samples to examine the effect of polydispersity on emulsion structure. Diffusive diffraction of the continuous phase showed that structural disorder could be induced by perturbing a matrix of monodisperse droplets with a number of droplets of a different size. Attempts to quantify the degree of structural perturbation using the mean self diffusion propagator were unsuccessful. However, qualitatively, this perturbation appears greatest when the number fraction of small droplets is high. 


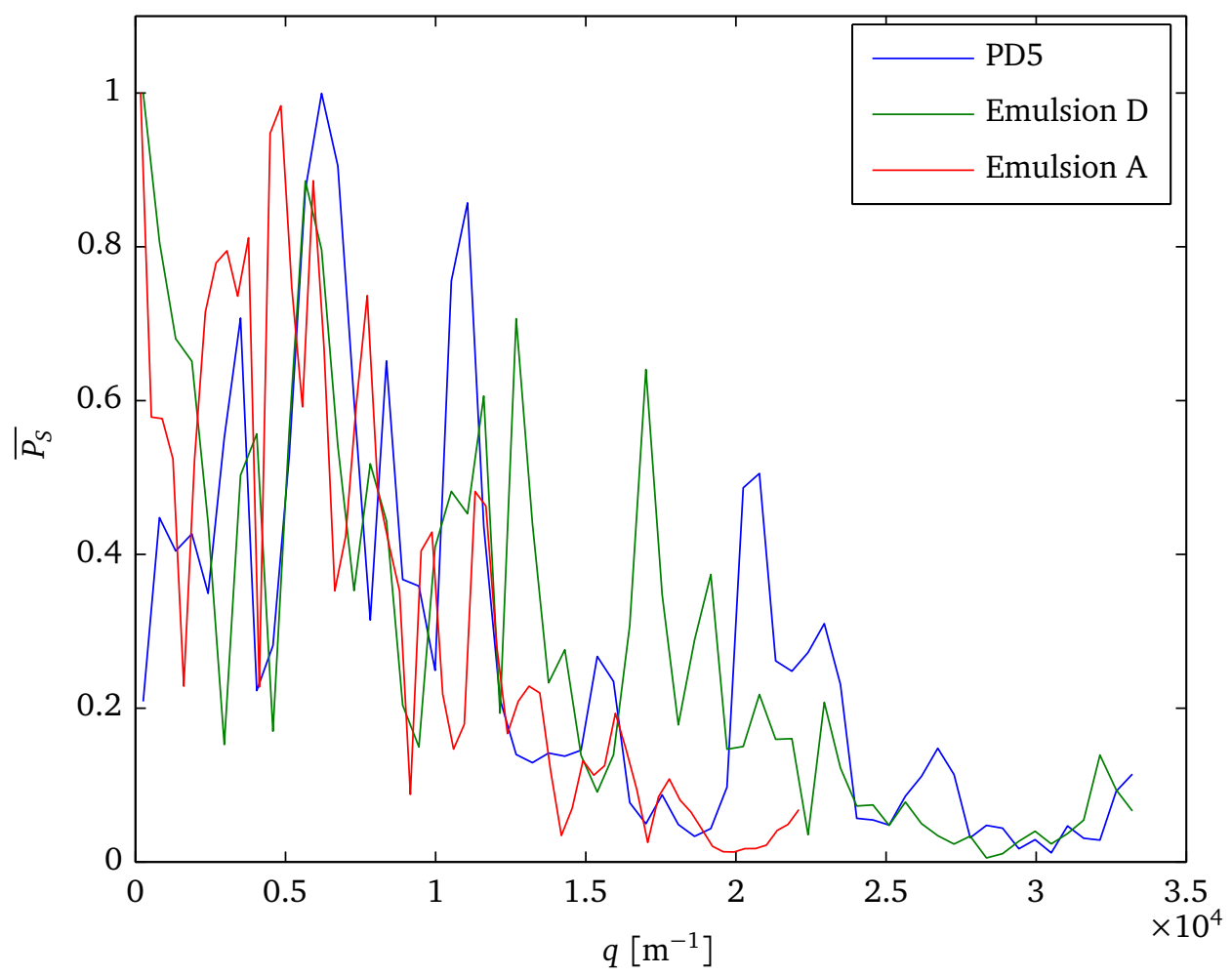

Figure 5.17: Normalised mean self diffusion propagators for PD5 and the two constituent emulsions. All measurements were made with $\Delta=1000 \mathrm{~ms}$.

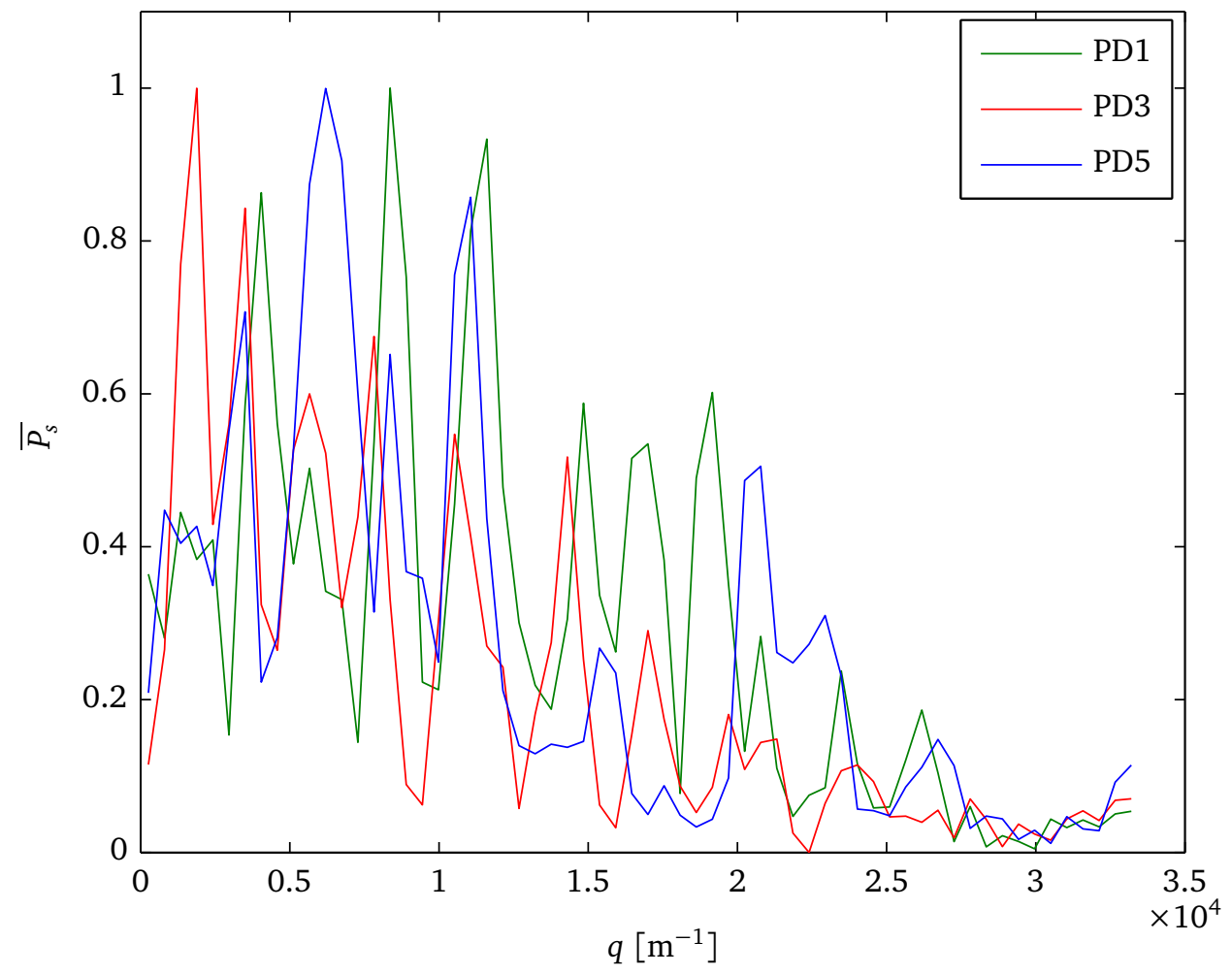

Figure 5.18: Normalised mean self diffusion propagators for three polydisperse emulsions. All measurements were made with $\Delta=1000 \mathrm{~ms}$. 



\section{6 \\ Emulsion rheology}

This chapter covers the rheological characterisation of monodisperse and bimodal emulsions using steady and oscillatory shear. The goal was to establish how the droplet size and polydispersity of the emulsions affected their non-Newtonian and viscoelastic properties, and whether the trends would be similar to those observed for monodisperse emulsions with much smaller droplet radii as has been reported in the literature.

\subsection{Steady shear}

\subsubsection{Experimental details}

All steady shear experiments were performed using a TA Instruments AR2000 controlled stress rheometer. Because the emulsion droplets were relatively large and the viscosity low, a $40 \mathrm{~mm}$ diameter acrylic parallel plate measuring geometry was used. The gap between the plates was set at $700 \mu \mathrm{m}$ to ensure multiple planes of droplets would be present in the sample. Larger gap sizes could not be used as the emulsion tended to settle to a low height under the influence of surface tension, preventing contact with the plate. The measuring geometry and sample were encased in a sealed solvent trap lined with wet tissues to slow the evaporation of the continuous phase as the experiments were performed at $293 \mathrm{~K}$.

Flow curves were performed from both high to low shear rates and in reverse between $1 \times 10^{-3}$ and $1 \times 10^{3} \mathrm{~s}^{-1}$. Shear rates below $1 \times 10^{-3} \mathrm{~s}^{-1}$ produced erratic stress responses and the sample was ejected from the geometry above $1 \times 10^{3} \mathrm{~s}^{-1}$. A peak hold was used for each discrete shear rate, and the shear rate was maintained long enough for the system to achieve an equilibrium stress response, assuming one existed, which generally meant that $t_{\text {hold }} \geq 1 / \dot{\gamma}$. The values reported for the stress responses are the mean of the equilibrium values, or the mean of a representative portion of the peak hold data if no equilibrium state existed. Because of the potential for droplet rupture and coalescence during flow, a new sample was used for each experiment. The bimodal emulsions were gently stirred for two minutes using a thin glass rod and left to settle for 10 minutes prior to characterisation. This ensured that the sample had a homogeneous distribution of each type of droplet and sufficient time to relax to an equilibrium state, therefore avoiding the measurement of unintentional thixotropic effects. 


\subsubsection{The effect of droplet size}

Flow curves for increasing and decreasing shear rates are shown in figures 6.1 and 6.2, respectively. Sample naming is as given in table 5.1. The emulsions produced relatively low stress responses for all shear rates, which can be attributed to the large droplet sizes and the resultant small Laplace pressures $p_{L}$, that provide less resistance to flow. In addition to the general low stress response, there were unexpected dependencies on the droplet size that are discussed below.

The results for emulsion $\mathrm{F}$ have been excluded from this chapter based on the seemingly erroneous results that were recorded. This sample was significantly more turbid in appearance than the other monodisperse emulsions. This increased level of light scattering indicates the presence of droplets much smaller than can be detected using optical microscopy or PGSE NMR using our experimental parameters. The presence of smaller droplets could be the consequence of non-linear behaviour induced by the use of high flow rates, or the rupture of droplets after formations due to the high velocity at which they are travelling. Characterisation using the static light scattering equipment available was not possible due to the deleterious effect the stirring motion of the machine has on the large droplets: if the experiment had shown the presence of smaller droplets, it would be uncertain as to whether they were present originally or the result of droplet breakup within the machine. Hence further investigation of this sample is required, the likelihood is that the sample was not monodisperse.

\subsubsection{Stress response}

Figure 6.1 shows the flow curves for all emulsions with increasing shear rates. The stress responses vary by as much as an order of magnitude at low shear rates and converge to approximately the same value at $1000 \mathrm{~s}^{-1}$. For emulsions B-F there is a stress plateau between approximately $1 \times 10^{-3}$ and $0.1 \mathrm{~s}^{-1}$ that can be attributed to either the presence of a yield stress or a period of shear banding [142]. This stress plateau is well defined for emulsions $B$ and $C$ and spans from $1 \times 10^{-3}$ to $0.1 \mathrm{~s}^{-1}$ at approximately $0.1 \mathrm{~Pa}$. For emulsions D-F the plateau appears to span an order of magnitude from $1 \times 10^{-3}$ to $0.1 \mathrm{~s}^{-2}$ at approximately $0.01 \mathrm{~Pa}$. Instead of a stress plateau, emulsion A produced a sharp minimum centred at approximately $6 \times 10^{-3}$.

Between approximately 0.1 and $10 \mathrm{~s}^{-1}$, all emulsions show shear thinning behaviour. Near $10 \mathrm{~s}^{-1}$ there is a shallow hump in the flow curve that represents either a transition to Newtonian flow behaviour or slippage at the wall of the rotor. A plot of the viscosity as a function of shear rate (figure 6.3) suggests that slippage is the cause, with the hump in the viscosity-shear rate plot similar to what has been observed in the literature [143].

Slippage involves the portion of the sample in contact with a surface having a finite velocity, and has been previously reported in emulsion systems [144-146]. True bulk flow data can typically be obtained by roughening the surfaces in contact with the sample [143-146]. However, flow curves obtained using a spiked steel parallel plate geometry produced erroneous data, as the large inertia of the plate could not be offset by the low viscosity of the monodisperse emulsions. Attempts to modify an acrylic plate with 100 grit sandpaper resulted in rupture of the droplets at moderate to high shear rates. Beyond $10 \mathrm{~s}^{-1}$ the samples were ejected from the geometry. 


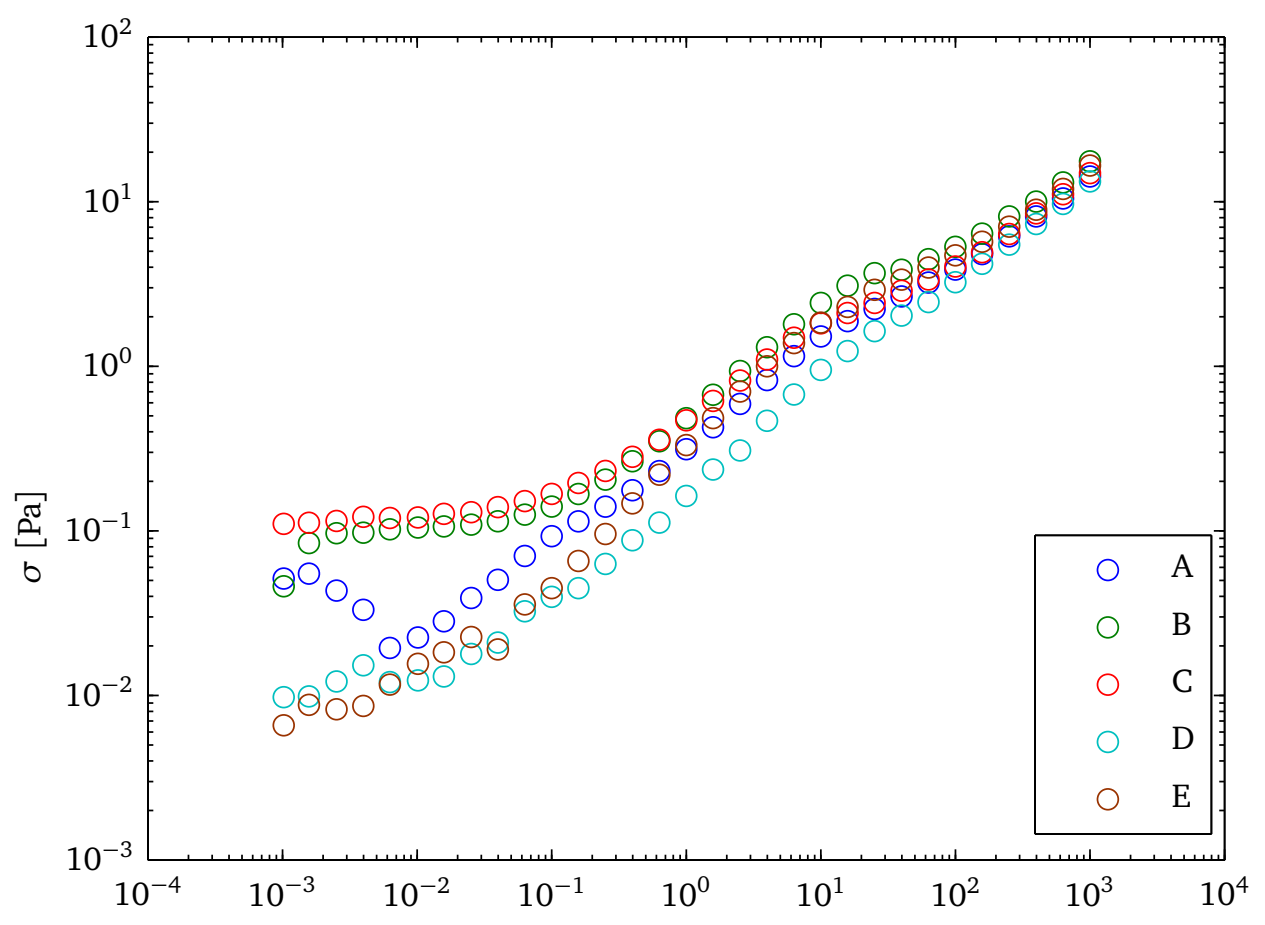

Figure 6.1: Flow curves for emulsions A-E measured from low to high shear rates.

\subsubsection{Stress growth and equilibrium}

Beyond the stress plateau, all samples reach an equilibrium stress response within seconds of applying the given shear rate. However, at lower shear rates $\left(<10^{-2} \mathrm{~s}^{-1}\right)$ the droplet size has a significant impact on the time dependent nature of the stress response. Larger droplets (emulsions A-C) show a gradual logarithmic accumulation of stress as the peak hold time elapses, eventually reaching an equilibrium value after approximately $700 \mathrm{~s}$, this time apparently being independent of the droplet size. In contrast, smaller droplets (emulsions D and E) produce an erratic stress response, not settling to an equilibrium value for times up to $1000 \mathrm{~s}$ (figure 6.4).

The gradual stress increase is the result of an initial viscoelastic response to the deformation and an equilibrium response is reached when the droplets have been maximally deformed [147]. Such behaviour has been previously observed in polydisperse emulsions $[148,149]$. The erratic stress response from the smaller droplets suggests they have much shorter relaxation times, or simply that the measurements were at the limit of the rheometer's capability.

\subsubsection{Degree of thixotropy}

Comparison of figures 6.1 and 6.2 shows that some emulsions have a degree of hysteresis in their flow behaviour. That is, they are thixotropic. An example of the hysteresis is shown in figure 6.5. Hysteresis in flow curves implies that the microand/or macrostructure are dependent on the strain history of the sample. Because the macrostructure has not changed between the experiments (there has been no flocculation, for example), the cause of the hysteresis is likely to be that the relaxation of the droplets to their equilibrium state is longer than the measurement time at each 


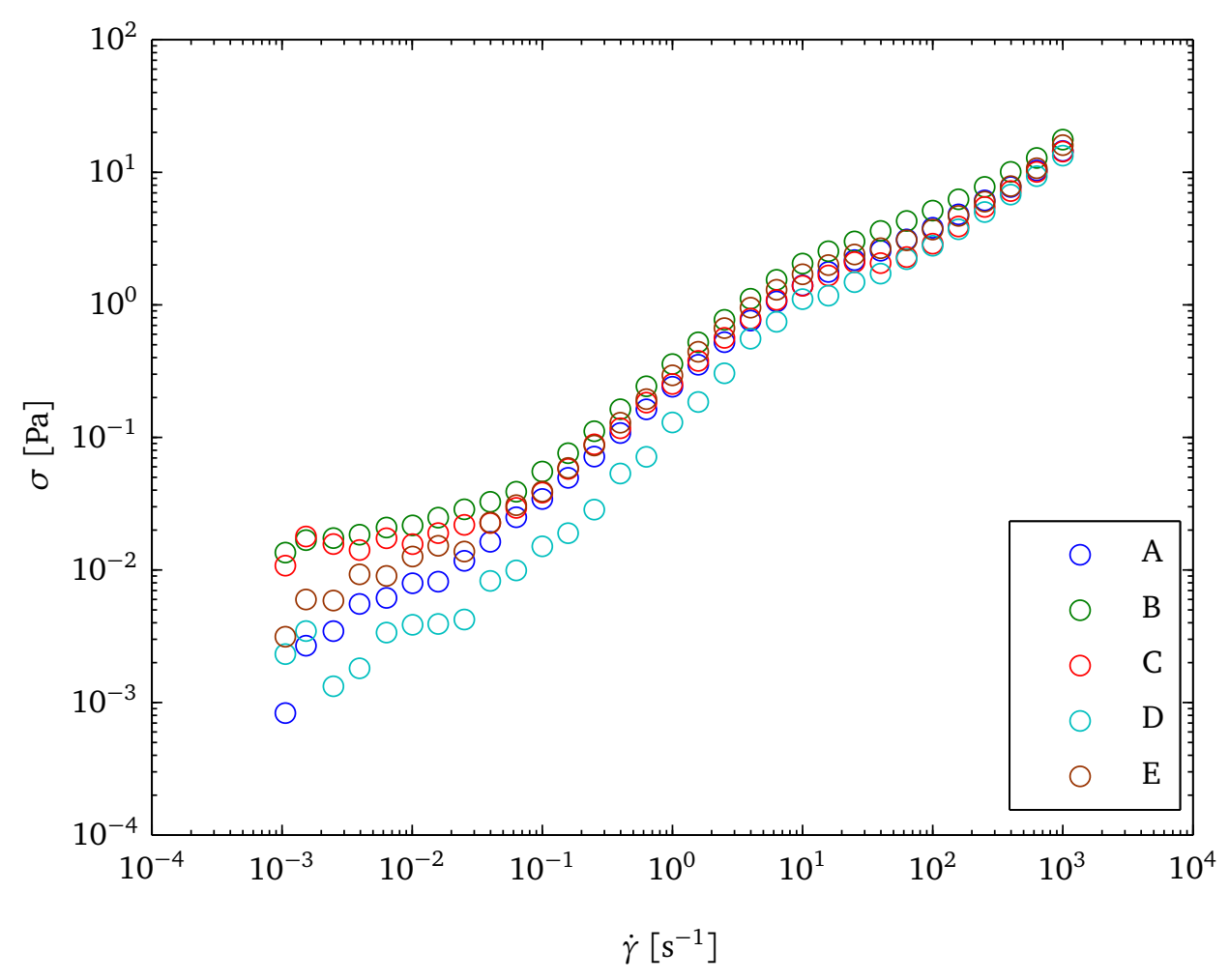

Figure 6.2: Flow curves for emulsions A-E measured from high to low shear rates.

shear rate the stress response differs. Attempts were made to determine the relaxation times for the emulsions using stress relaxation tests. Unfortunately, erratic results were obtained as the stress response was near the lower limits of the rheometer.

Using trapezoidal numerical integration, it was possible to establish the degree of thixotropy in the five emulsions studied by subtracting the area under the reverse curve from the area beneath the forward flow curve. Emulsions A, B and C show some degree of thixotropy, while emulsions $\mathrm{C}$ and $\mathrm{D}$ show virtually none. While the relatively small number of data point (31) and uncertainty in the low shear rate data limited the quantitative value of these calculations, it suggests that there are two modes of relaxation behaviour within the five emulsions.

\subsubsection{The effect of polydispersity}

\subsubsection{Stress response}

Flow curves for the five bimodal emulsions are given in figure 6.6 for low to high and figure 6.7 for high to low shear rates. The polydispersity has not significantly altered the bulk flow characteristics of the emulsions compared to their monodisperse constituents (figure 6.6 and 6.7). The bimodal emulsions generally have a stress plateau below approximately $0.1 \mathrm{~s}^{-1}$ and are shear thinning at higher shear rates.

Figure 6.6 shows that the bimodal emulsions may be distinguished only within the stress plateau region. The addition of a small amount of emulsion $\mathrm{D}\left(x_{D}=0.1\right)$ seems to preserve the wall effect observed in emulsion A, which is no longer present as the number fraction increases to $x_{D}=0.25$. At $x_{D}=0.5$ and above it becomes difficult to discriminate between the samples. 


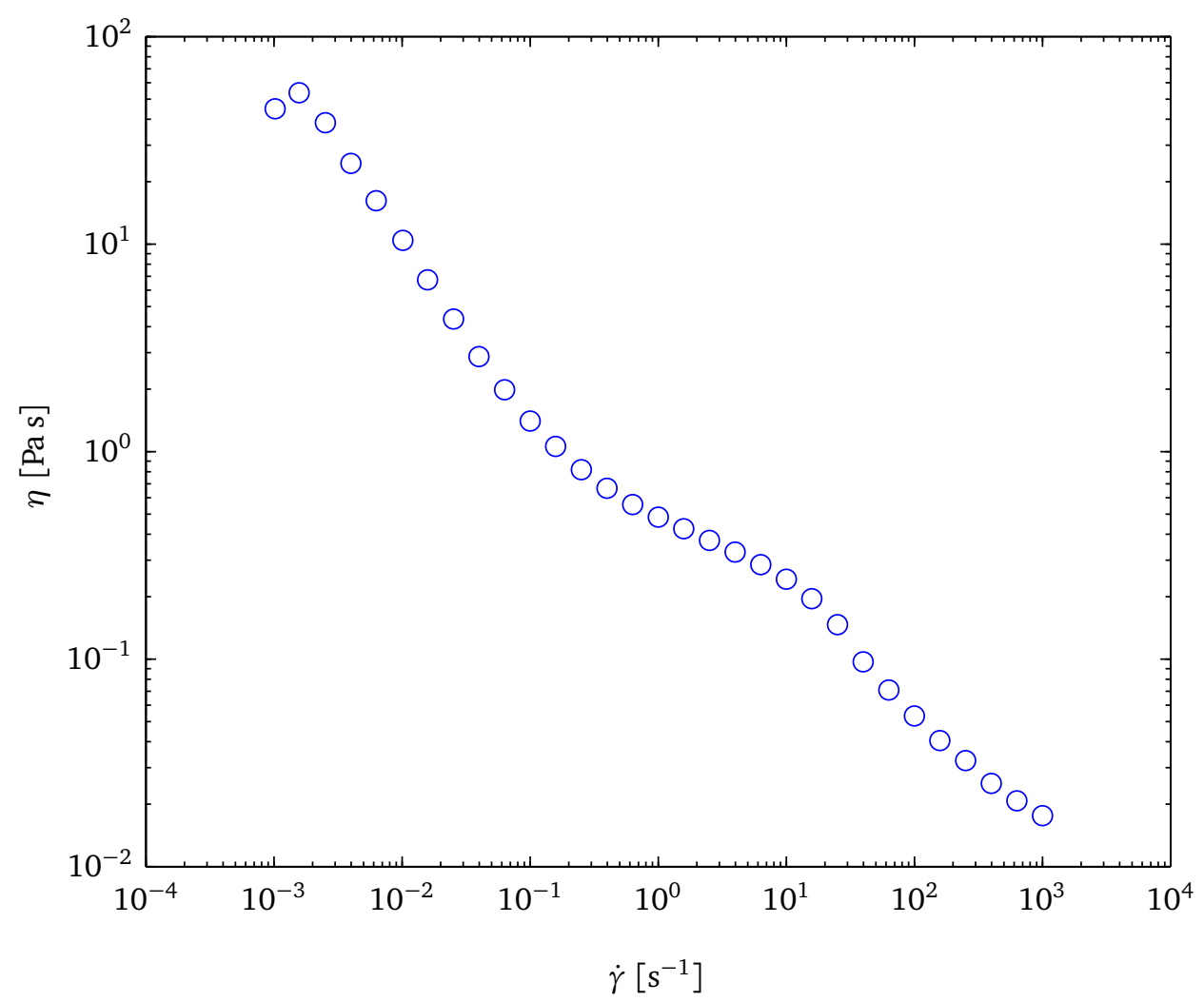

Figure 6.3: A plot of the viscosity as a function of shear rate for emulsion B, which is representative of the behaviour shared by all emulsions. The hump centred at approximately $10 \mathrm{~s}^{-1}$ and the continued decline of the viscosity suggests that there is slippage occurring within the sample [143].

When the flow curve is measured from high to low shear rates (figure 6.7), the flow curves are indistinguishable and similar to the response generated by emulsion A. When the response of the monodisperse emulsions is considered, this suggests that the larger droplets dominate the steady shear flow behaviour from high to low shear rates, as the stress response only differs significantly when a critical droplet size is reached. The disrupted droplet packing has not made the emulsions more resistant to deformation.

\subsubsection{Stress growth and equilibrium}

As discussed in section 6.1.2.2, at low shear rates monodisperse emulsions with larger droplets generate a stress response that increases logarithmically with time to an eventual equilibrium value. Conversely, emulsions with smaller droplets show an erratic stress response that appears to be largely time invariant. Such a logarithmic variation of the stress response with time appears to be correlated with the existence of a stress plateau within the flow curve at low shear rates. This effect is observed irrespective of whether a bimodal or monodisperse emulsion is considered. Additional samples would need to be analysed to confirm this correlation.

\subsubsection{Degree of thixotropy}

Two of the polydisperse samples displayed thixotropic flow behaviour: PD1 and PD2. The magnitude of the thixotropy for PD1 was similar to that of emulsion A, suggesting that the relatively low number of smaller droplets does not significantly alter the 


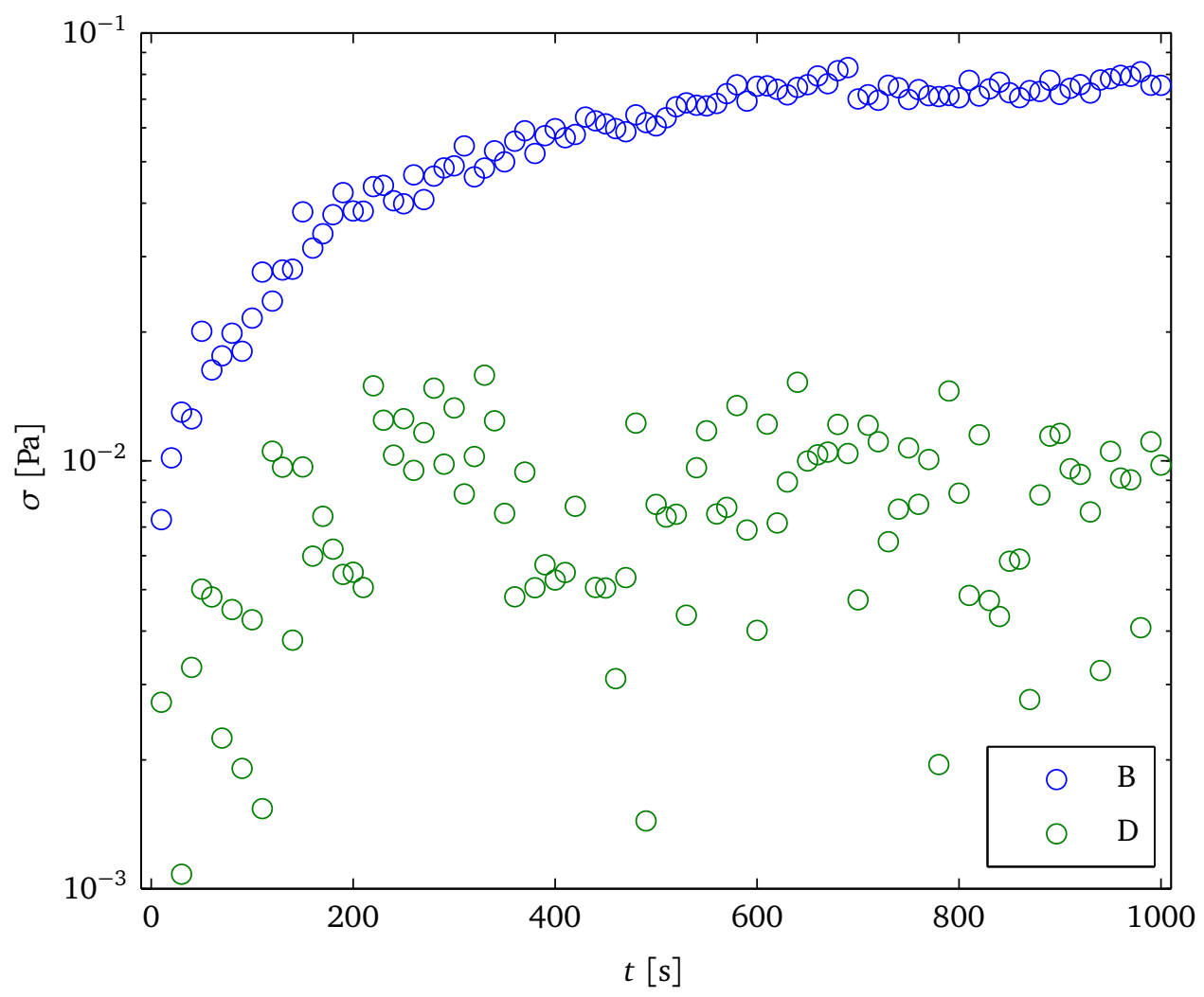

Figure 6.4: The time dependent stress behaviour for emulsions B and D for a shear rate of $1 \times 10^{-3} \mathrm{~s}^{-1}$, which are representative of the smooth logarithmic stress growth observed for larger droplets and the erratic response detected for the smaller droplets.

relaxation of large droplets to an equilibrium shape. When $x_{D}=0.25$, the degree of thixotropy surpasses that of emulsion A. At higher number fractions of emulsion A the bimodal emulsions do not show any thixotropic effects, which may be the result of the structure constraining the shape of the larger droplets or there simply may not be a sufficient amount of large droplets to produce a measurable effect.

\subsection{Oscillatory shear}

\subsubsection{Experimental details}

Oscillatory shear experiments were performed using a TA Instruments AR2000 controlled stress rheometer with the motor set to controlled strain mode and using the same parallel plate geometry, solvent trap and operating temperature described in section 6.1.1.

Strain sweep experiments were performed for strains between $1 \times 10^{-3}$ and 10 . Measurements below $\gamma=1 \times 10^{-3}$ produced erratic results even when the mean value for a large number of oscillations was taken. The emulsions typically behaved as a Newtonian liquid $\left(\delta=90^{\circ}\right)$ at strains above 10, making additional measurements unnecessary. For each strain, two "dummy" oscillations were performed on the sample where no measurements were made, followed by five "proper" oscillations whose measurements were averaged to give values for $G^{\prime}, G^{\prime \prime}$ and $\delta$. Additional oscillations provided diminishing returns on the quality of the measurements when weighed 


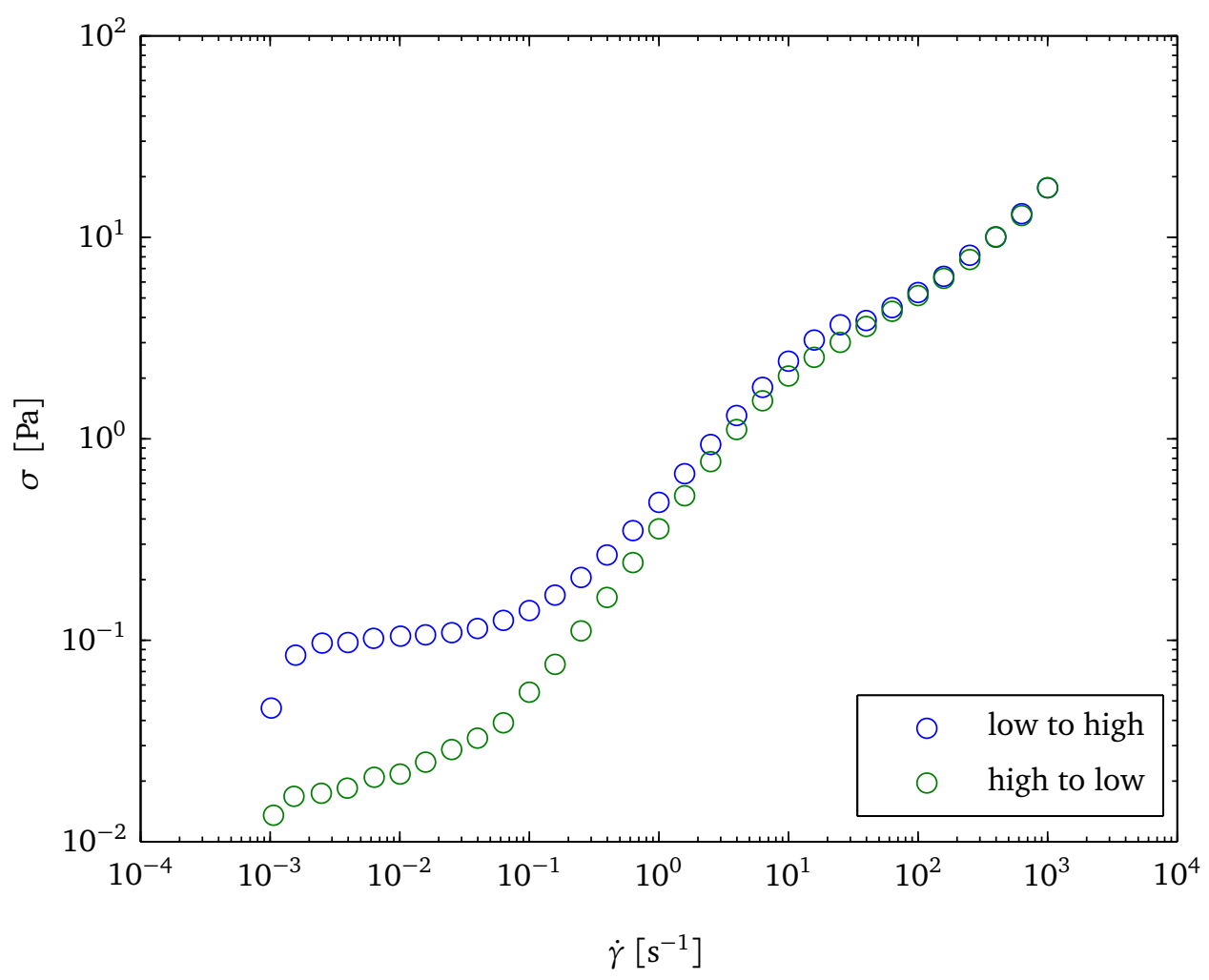

Figure 6.5: Flow curves for emulsion B that demonstrate the general behaviour shown by all monodisperse emulsions: a stress plateau, shear thinning with possible slippage and thixotropy when the sample is subjected to increasing and decreasing shear rates.

against the time required. Experiments were performed at $0.1,1$ and $10 \mathrm{rads}^{-1}$, where 0.1 and 10 rads $^{-1}$ represented the minimum and maximum values used in subsequent frequency sweeps.

After the linear viscoelastic (LVE) range for a sample had been established, frequency sweeps between 0.1 and $10 \mathrm{rads}^{-1}$ were performed at an appropriate strain value. The same number of dummy scans and measurement scans were made as described above. The rotational frequencies were limited to $0.1 \mathrm{rad} \mathrm{s}^{-1}$ by the sensitivity of the instrument and $10 \mathrm{rad} \mathrm{s}^{-1}$ due to the Newtonian behaviour of the sample (see above).

\subsubsection{The effect of droplet size}

\subsubsection{Strain sweeps}

Plots of the shear moduli $G^{\prime}$ and $G^{\prime \prime}$ as a function of strain amplitude are shown in figure 6.8 and 6.9, respectively. At low strains, there is a small LVE region between approximately $\gamma=1 \times 10^{-3}$ and $3 \times 10^{-3}$, where the moduli are approximately independent of the strain amplitude. This region of linearity is more pronounced for the loss modulus. Beyond the LVE region, the storage modulus decreases several orders of magnitude as the strain amplitude approaches its maximum value of 10, whereas the loss modulus decreases less than an order of magnitude.

Figure 6.8 shows that emulsions A-C have very similar elastic responses, and only once the droplets become smaller does the storage modulus appear to increase within the LVE range (between approximately $\gamma=10^{-3}$ and $10^{-2}$ for all emulsions). At high 


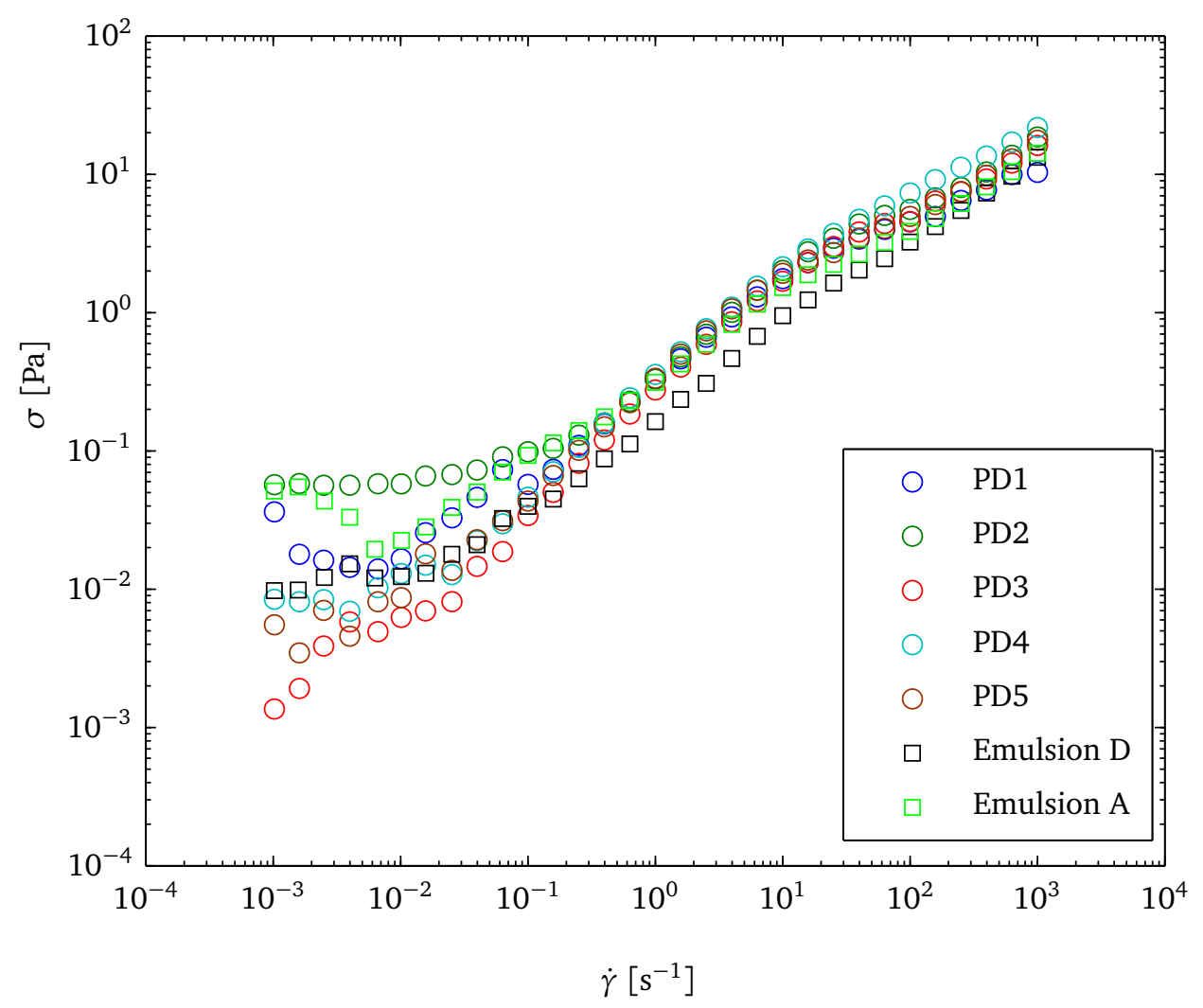

Figure 6.6: Flow curves for all bimodal emulsions and constituent monodisperse emulsions performed from low to high shear rates.

strains the emulsions converge to nearly the same value.

Figure 6.9 shows the loss modulus for each emulsion. Unlike in figure 6.8, there is no obvious trend in how $G^{\prime \prime}$ varies with droplet size. The crossover point where $G^{\prime}=G^{\prime \prime}$ is the strain amplitude at which the emulsions transition from being predominately elastic to primarily viscous. There was no trend observed for this transition point with changing droplet size.

The proportion of elastic and viscous responses is measured by $\delta$. In table 6.1, $\delta$ is given for each monodisperse emulsion within its LVE range. All emulsions approach $\delta=90^{\circ}$ at high strains. This combined with the data in table 6.1 shows that these emulsions are locally primarily elastic, while their bulk flow behaviour is predominately viscous. The monodisperse emulsions studied by Mason et al. $[10,124,125]$ showed similar trends, but with larger moduli and less reduction of the storage modulus with strain amplitude. This can be attributed to the smaller droplet size and increased disperse phase viscosity.

Table 6.1: A comparison of $\delta$ for all emulsions

\begin{tabular}{cccccc}
\hline Emulsion & A & B & C & D & E \\
\hline$\delta\left[^{\circ}\right]$ & 40 & 25 & 23 & 21 & 17 \\
\hline
\end{tabular}

Increasing the rotational frequency of the strain sweeps reduced the elastic character of the emulsion at low strain amplitudes at $10 \mathrm{rad} \mathrm{s}^{-1}$ where all emulsions were primarily 


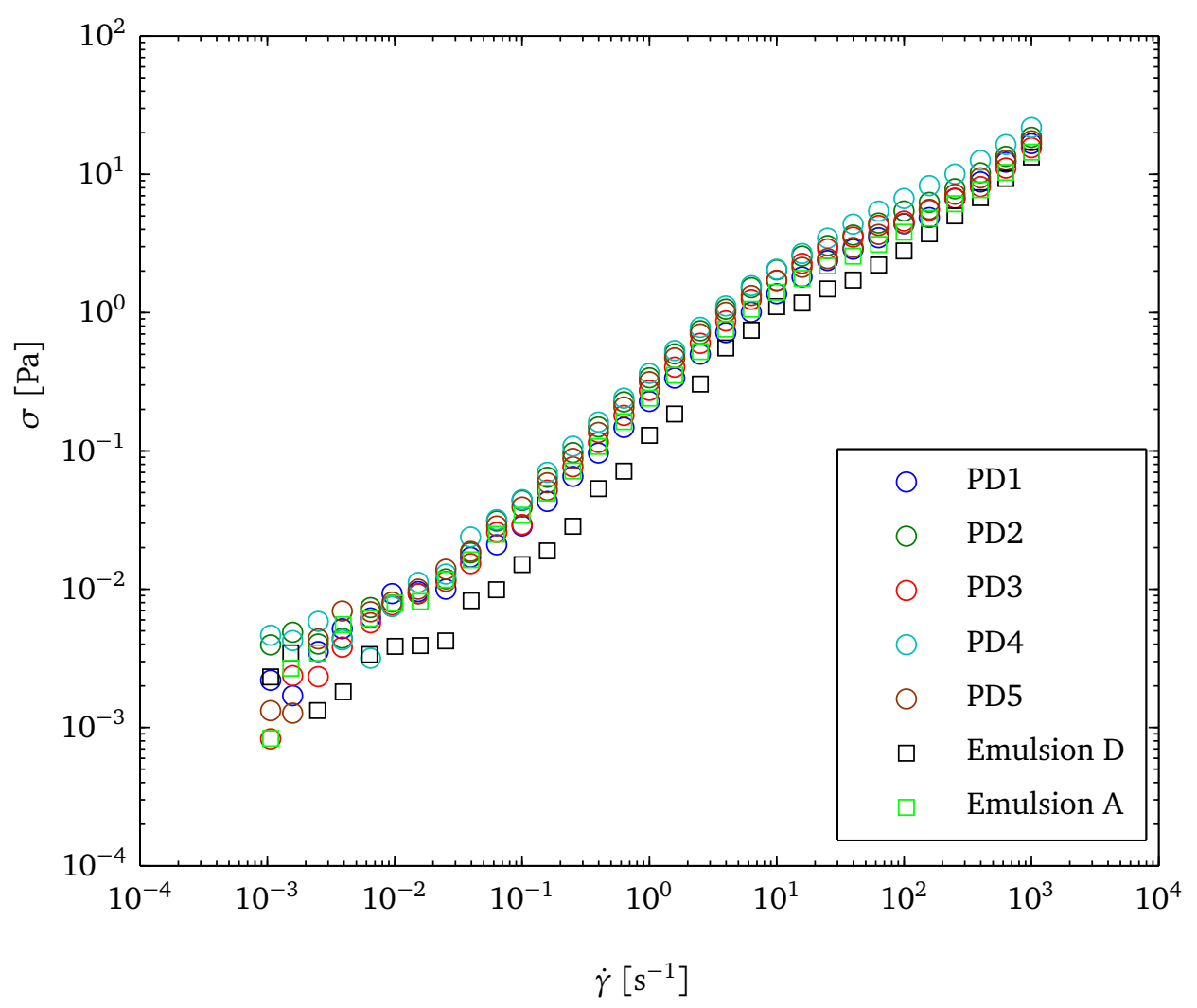

Figure 6.7: Flow curves for all bimodal emulsions and constituent monodisperse emulsions performed from high to low shear rates.

viscous for all strain amplitudes. The distinction between the monodisperse samples at $0.1 \mathrm{rad} \mathrm{s}^{-1}$ did not justify the ten-fold increase in experimental time over the $1 \mathrm{rad} \mathrm{s}^{-1}$ experiments that are reported here. However, for the bimodal emulsions, the data reported are from experiments performed at $0.1 \mathrm{rad} \mathrm{s}^{-1}$ so that differences between the sample responses at low strain amplitudes and oscillatory frequencies are emphasised.

\subsubsection{Frequency sweeps}

Given that the LVE range for each emulsion was small and at low strains, obtaining reliable frequency sweeps was difficult. Plots for $G^{\prime}$ and $G^{\prime \prime}$ as a function of angular frequency are shown in figure 6.10 and 6.11, respectively. Each emulsion shows an increase of the storage modulus with an increase in the angular frequency. That is, the emulsions show increasing rigidity at shorter time periods. Emulsions A-C generate practically identical responses, with D-F showing an increased elastic response.

Inspection of figure 6.11 shows that the loss modulus increases more rapidly with angular frequency than the storage modulus does and that the behaviour for all emulsions is nearly identical.

To quantify the onset of primarily viscous behaviour, the crossover point was measured for each emulsion and is shown in table 6.2. The results show that emulsions A$\mathrm{C}$ have crossover points at similar stresses, while D and E behave differently. The crossover point for emulsion $\mathrm{D}$ is at a higher stress and frequency, while emulsion $\mathrm{E}$ is primarily elastic for all rotational frequencies. A reason for this could be that the increasing Laplace pressure and associated reduction in droplet relaxation time allows 


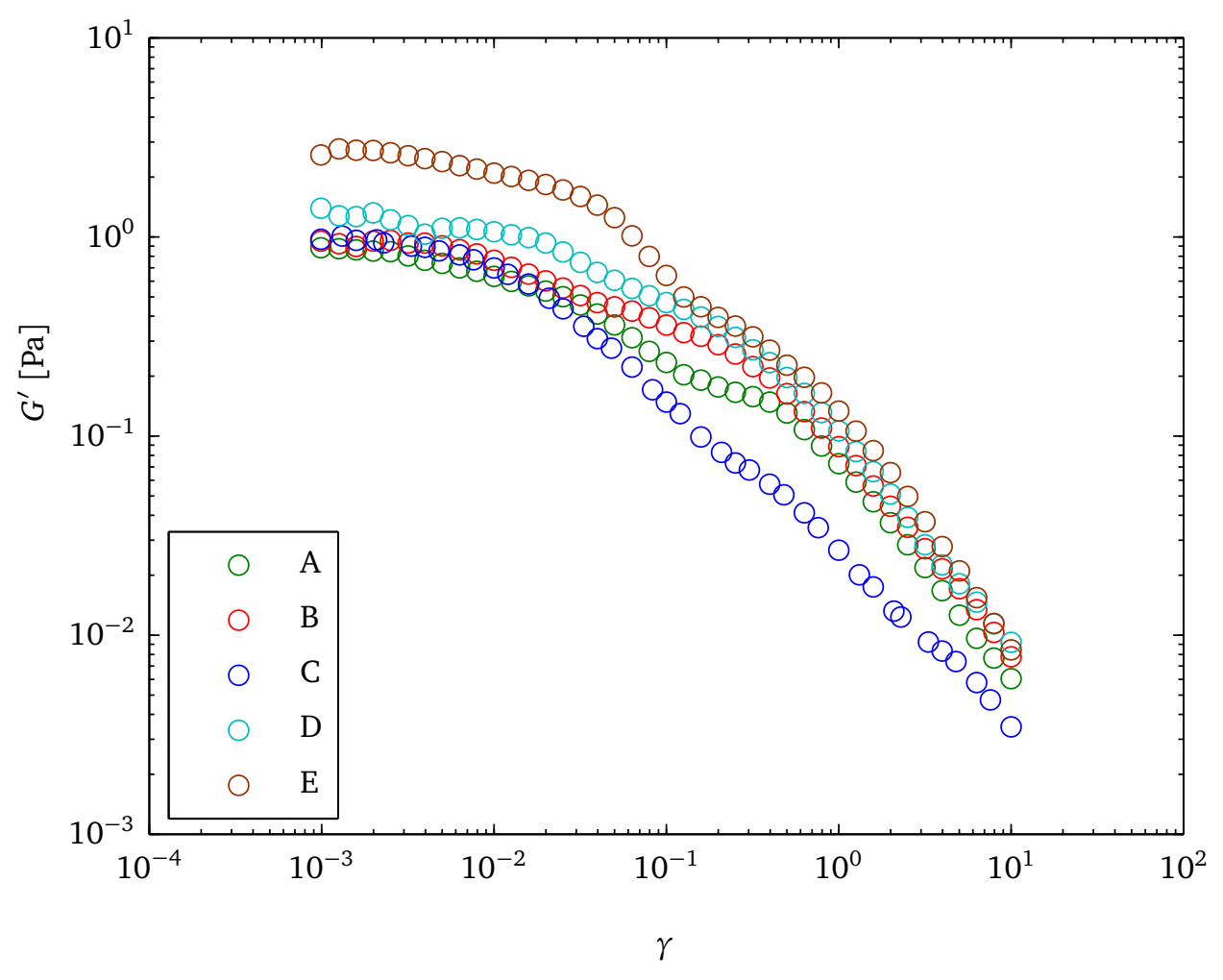

Figure 6.8: $G^{\prime}$ for all emulsions at $\omega=1 \mathrm{rad} \mathrm{s}^{-1}$.

the droplets to "keep up" with the oscillation and avoid dissipating a majority of the acquired energy. A gradual increase in $G^{\prime}$ coupled with a more rapid raise in $G^{\prime \prime}$ is similar to the behaviour observed by Mason [8] using diffusing wave spectroscopy with concentrated emulsions consisting of sub-micrometre droplets.

Table 6.2: A table of the strain and modulus values when $G^{\prime}=G^{\prime \prime}$ for all emulsions.

\begin{tabular}{ccc}
\hline Emulsion & $\boldsymbol{\omega}\left[\mathrm{rad} \mathrm{s}^{-1}\right]$ & $\boldsymbol{G}_{\text {cross }}[\mathrm{Pa}]$ \\
\hline $\mathrm{A}$ & 1 & 0.9 \\
$\mathrm{~B}$ & 1 & 0.6 \\
$\mathrm{C}$ & 5 & 0.9 \\
$\mathrm{D}$ & 8 & 2.4 \\
$\mathrm{E}$ & - & - \\
\hline
\end{tabular}

\subsubsection{The effect of polydispersity}

\subsubsection{Strain sweeps}

Figure 6.12 shows the storage moduli for each bimodal emulsion and the constituent monodisperse samples. Much like the steady shear data, PD1 shows a similar response to emulsion A. As the number fraction of emulsion D is increased in PD2 and PD3, the storage modulus decreases by an order of magnitude. Further increasing the emulsion D number fraction results in the response being similar to PD2 and PD3 at large strain amplitudes but corresponding to the constituent emulsions at low strain amplitudes. At higher strain amplitudes, $G^{\prime}$ is four to five orders of magnitude lower than within 


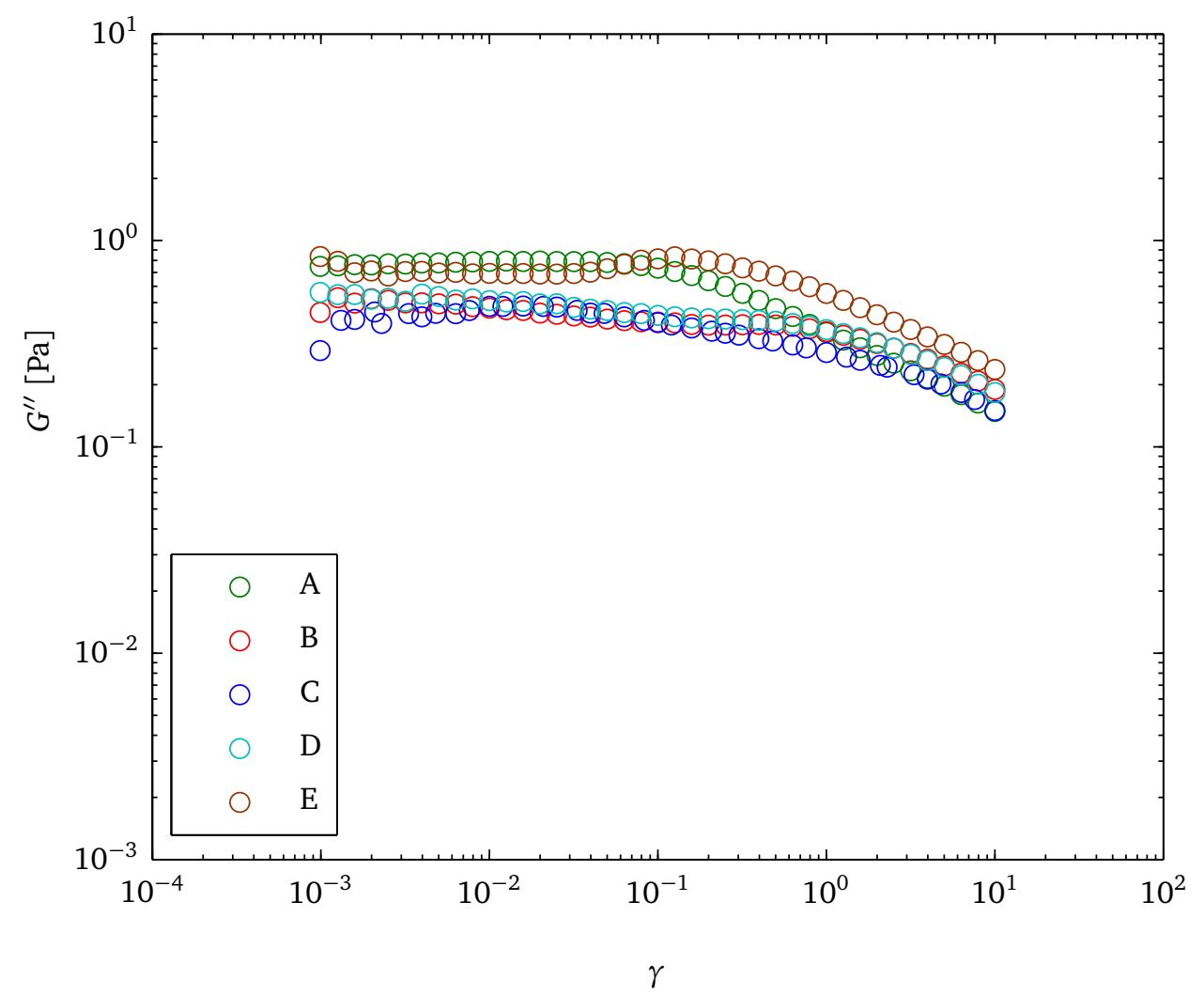

Figure 6.9: $G^{\prime \prime}$ for all emulsions at $\omega=1 \mathrm{rad} \mathrm{s}^{-1}$.

the LVE range.

The loss modulus for each bimodal emulsion is shown in figure 6.13. In contrast to the storage modulus, $G^{\prime \prime}$ changes very little as the strain amplitude is increased, and is nearly identical for each bimodal emulsion. This common viscous dissipation does not resemble the loss modulus of either emulsion A or D.

The crossover point for the bimodal emulsions shows a similar dependence on the polydispersity as is shown by the storage modulus, where the behaviour is split into three groups. PD1; PD2 and PD3; PD4 and PD5. With the anomalous viscous response shown by emulsion A during strain sweeps (figure 6.9), it is difficult to make meaningful comparisons with the constituent emulsions.

\subsubsection{Frequency sweeps}

Frequency sweeps were performed at $\gamma=1 \times 10^{-3}$, which represents a strain amplitude at which all emulsions display linear viscoelastic behaviour (see figure 6.12). In figures 6.14 and 6.15 are shown the variation of $G^{\prime}$ with angular frequency for each bimodal emulsion.

The storage moduli for PD1, PD2 and PD3 align with the results discussed above, where a small perturbation has little effect on the viscoelastic behaviour, while increasing the proportion of emulsion D creates a response that differs significantly from either constituent emulsion (figure 6.14). As $x_{D}$ is increased to 0.75 and 0.9 , the storage modulus is identical to that of emulsion A (figure 6.15).

In figure 6.16 is shown the variation of $G^{\prime \prime}$ with angular frequency. It is evident 


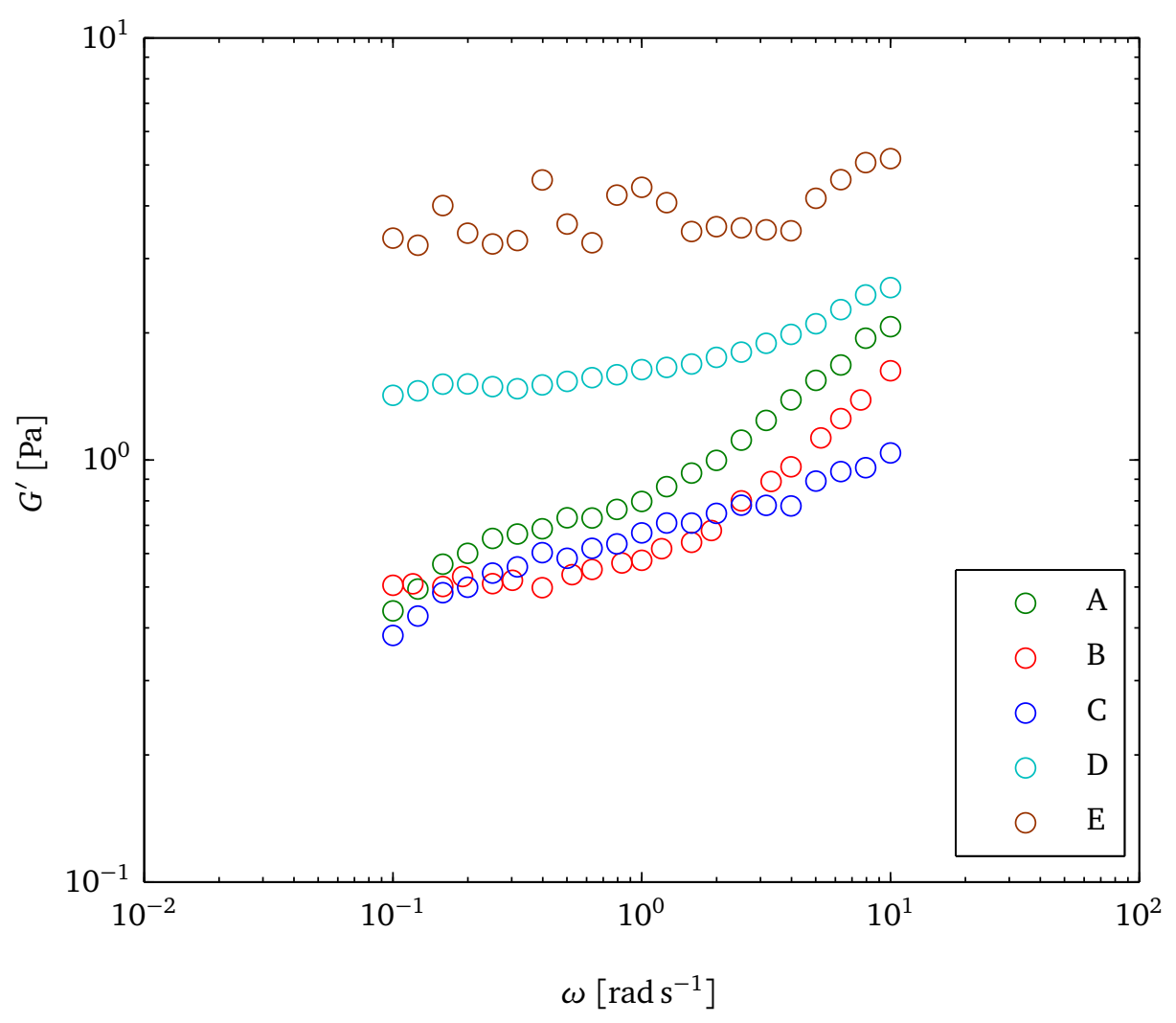

Figure 6.10: $G^{\prime}$ for all emulsions at $\gamma=1 \times 10^{-3}$.

that it varies in a manner akin to that of the monodisperse emulsions, where the loss modulus is essentially independent of emulsion polydispersity. Regardless of the droplet packing, the energy dissipated by the emulsions monotonically increases from a very small amount at low angular frequencies to a value sufficient to render the emulsion predominately liquid-like at high frequencies.

The crossover points for the bimodal emulsions show a trend similar to that obtained from the strain sweep data: PD1, PD4 and PD5 transition to predominately viscous responses at $\omega \approx 1 \mathrm{rad} \mathrm{s}^{-1}$, whereas the change occurs at approximately one order of magnitude less $\left(\omega \approx 0.2 \mathrm{rad} \mathrm{s}^{-1}\right)$ for PD2 and PD3.

\subsection{Discussion}

\subsubsection{General considerations}

All of our monodisperse and bimodal emulsions show many of the characteristics associated with soft glassy materials: potential yielding at low shear rates followed by shear thinning as the shear rate is increased, and viscoelastic moduli that are weakly dependent on the rotational frequency [150]. This behaviour is the result of mesoscopic structural disorder, metastability and slow internal dynamics $[150,151]$. In general, the energy required for rearrangements and relaxation in these systems greatly exceeds thermal energy at standard conditions [150]. At low stress values, the elements within the structure are trapped in a metastable and disordered state. Beyond the yield stress, these elements can cross local energy barriers and the sample flows [152]. 


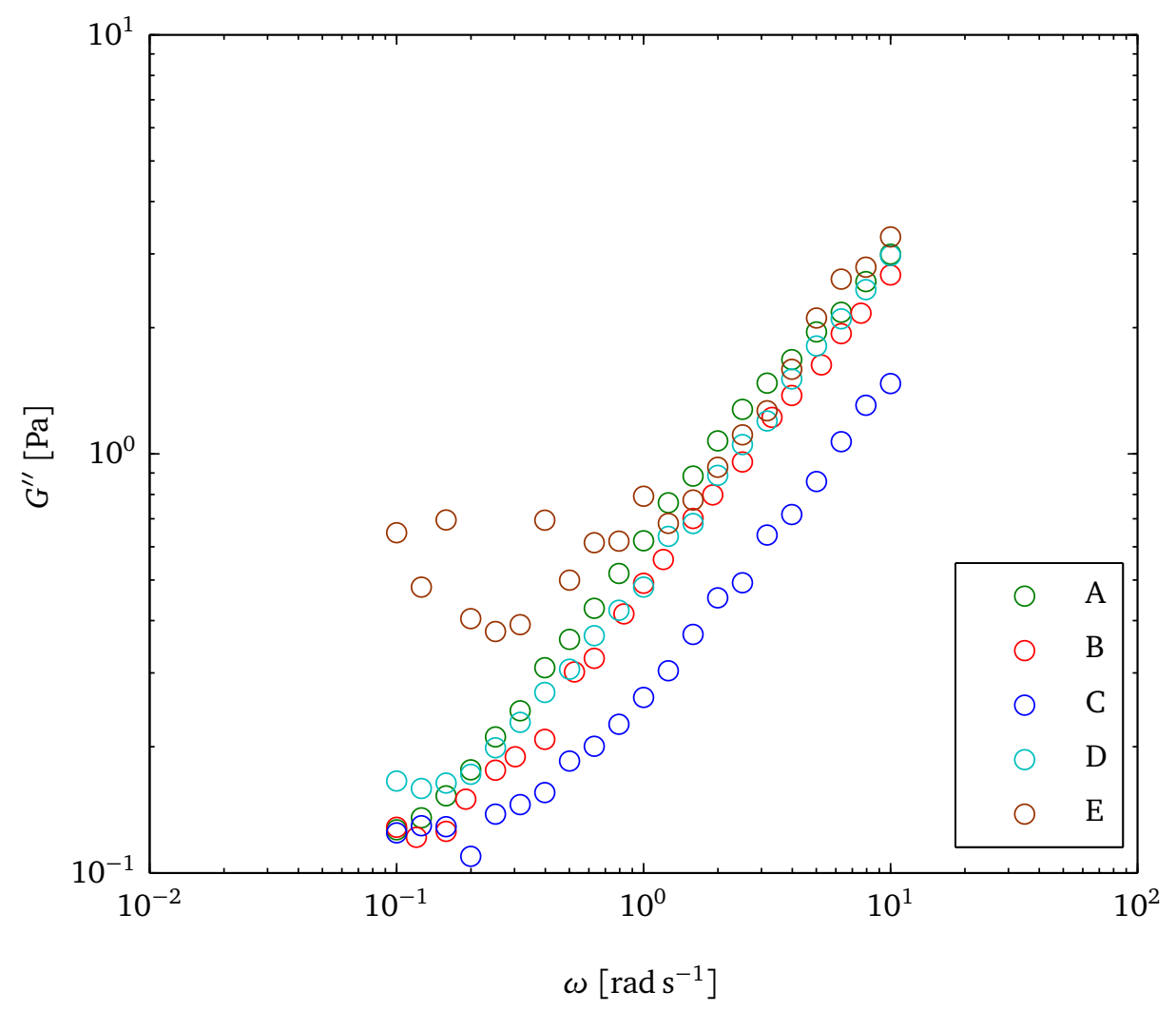

Figure 6.11: $G^{\prime \prime}$ for all emulsions at $\gamma=1 \times 10^{-3}$.

It was established in chapter 5 that $\phi \approx 0.74$ for the monodisperse emulsions and approximately 0.80 for the bimodal samples. At this point, the system is said to be beyond the jamming transition, where the droplets have some degree of crystallinity (they are "glassy") [153]. This crystallinity in the structure imparts yielding properties on the emulsion, which will not flow until the structure is perturbed sufficiently. This is reflected in figure 6.1. The soft and glassy behaviour is quantified using the concept of "effective temperature" $x$, of the system [150]. It essentially describes how readily the system is able to rearrange into new metastable states and flow, and is a consequence of the interactions between the particles in the system. If the effective temperature of the system is greater than the energy well of the current metastable state $x_{g}$, then the system is above the glass transition temperature and flows with shear thinning properties. Conversely, if $x \leq x_{g}$ then the system is at or below the glass transition temperature and shows yielding behaviour.

A soft glassy material then has the following relationships [150]:

$$
\begin{aligned}
G^{\prime} & \propto \omega^{2}(x>3), \\
G^{\prime} & \propto \omega^{x-1}(1<x<3), \\
G^{\prime \prime} & \propto \omega(x>2), \\
G^{\prime \prime} & \propto \omega^{x-1}(1<x<2)
\end{aligned}
$$




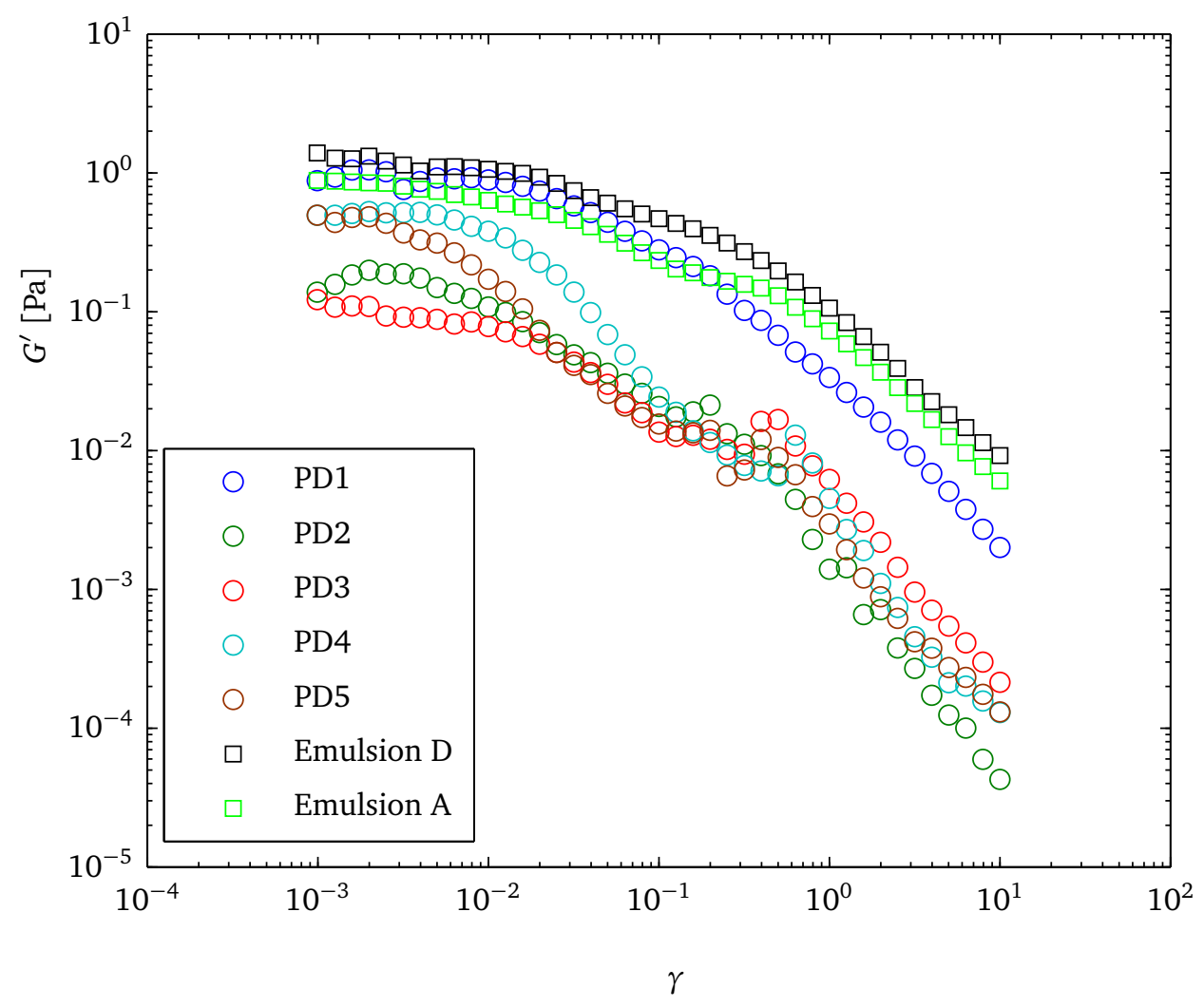

Figure 6.12: Strain sweep storage moduli for the bimodal emulsions compared to emulsions A and D performed at $0.1 \mathrm{rads}^{-1}$.

and

$$
\begin{aligned}
& \sigma \propto \dot{\gamma}^{x-1}(1<x<2), \\
& \sigma=\sigma_{y}(x \leq 1)
\end{aligned}
$$

Upon fitting flow curves and frequency sweeps to both poly- and monodisperse emulsions, it was found that for all systems $x \approx 1.6$ regardless of droplets size and polydispersity. This means that all emulsions are above the glass transition temperature, so flow readily. Additionally, within our domain of droplet sizes neither the size of the droplet nor its packing significantly affects its ability to rearrange into a new metastable state up application of shear forces.

The yield stress, as well as the stress response at higher shear rates, for these emulsions is between $10^{-2}$ and $10^{1} \mathrm{~Pa}$, which is low compared to many colloidal systems [154] and suggests the energy minima each metastable state represents is shallow. This can be rationalised by considering that the variables that significantly influence how readily an interface is deformed: the Laplace pressure [124]:

$$
P_{L}=\frac{2 \xi}{a}
$$

and the viscosities of the continuous and dispersed phases. Both are low, relative to conventional emulsion systems, in these samples. Using emulsion $\mathrm{E}$ as an example, droplets of dodecane with a radius of $30 \mu \mathrm{m}$ dispersed through a solution of $5 \mathrm{wt}$. 


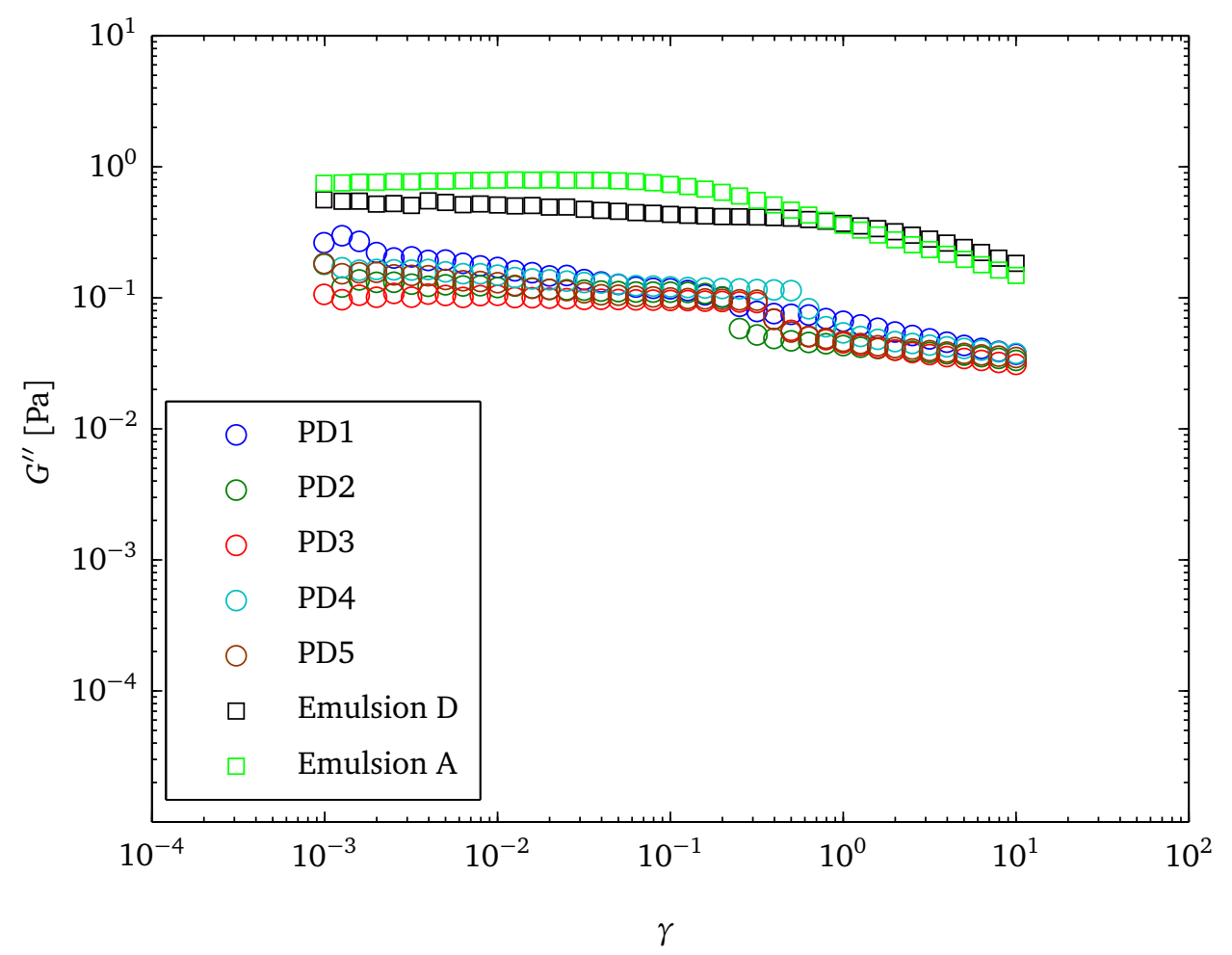

Figure 6.13: Strain sweep loss moduli for the bimodal emulsions compared to emulsions A and D performed at $0.1 \mathrm{rad} \mathrm{s}^{-1}$.

\% Pluronic F127 have a Laplace pressure of approximately $530 \mathrm{~Pa}$, which is several orders of magnitude lower than the 100 to $1000 \mathrm{kPa}$ pressures generated by the lowto sub-micrometre droplets typically studied in the literature. This combined with the low viscosities of dodecane and water results in the low yield stresses.

It is also possible that the stress plateaus are not the consequence of a yield stress, but shear banding, which involves regions within the sample experiencing different velocity gradients. Shear banding has been observed in emulsions previously [155, 156]. The velocity profile of the sample under shear can be characterised using rheo NMR [157]. Unfortunately rheo NMR could not be used to characterise these emulsions as their size prevents them fitting within the confines of the concentric cylinder and cone and plate geometries typically utilised in such experiments.

Beyond the plateau region, all emulsions behave similarly and converge to nearidentical stress responses at $1000 \mathrm{~s}^{-1}$. This implies that at large strains the microstructure for each emulsion is essentially indistinguishable. Again, this is likely the result of the low Laplace pressures and viscosity of the emulsion droplets. At such low Laplace pressures, there is insufficient resolution to distinguish each stress response beyond the bounds of experimental uncertainty.

\subsubsection{Monodisperse emulsions}

The value of the stress response at the plateau scales unusually with the droplet size. Based on the notion that the Laplace pressure controls droplet deformation, we would expect the smallest droplets to generate the highest stress responses within the plateau. However, it appears that the Laplace pressure can be used to broadly characterise 


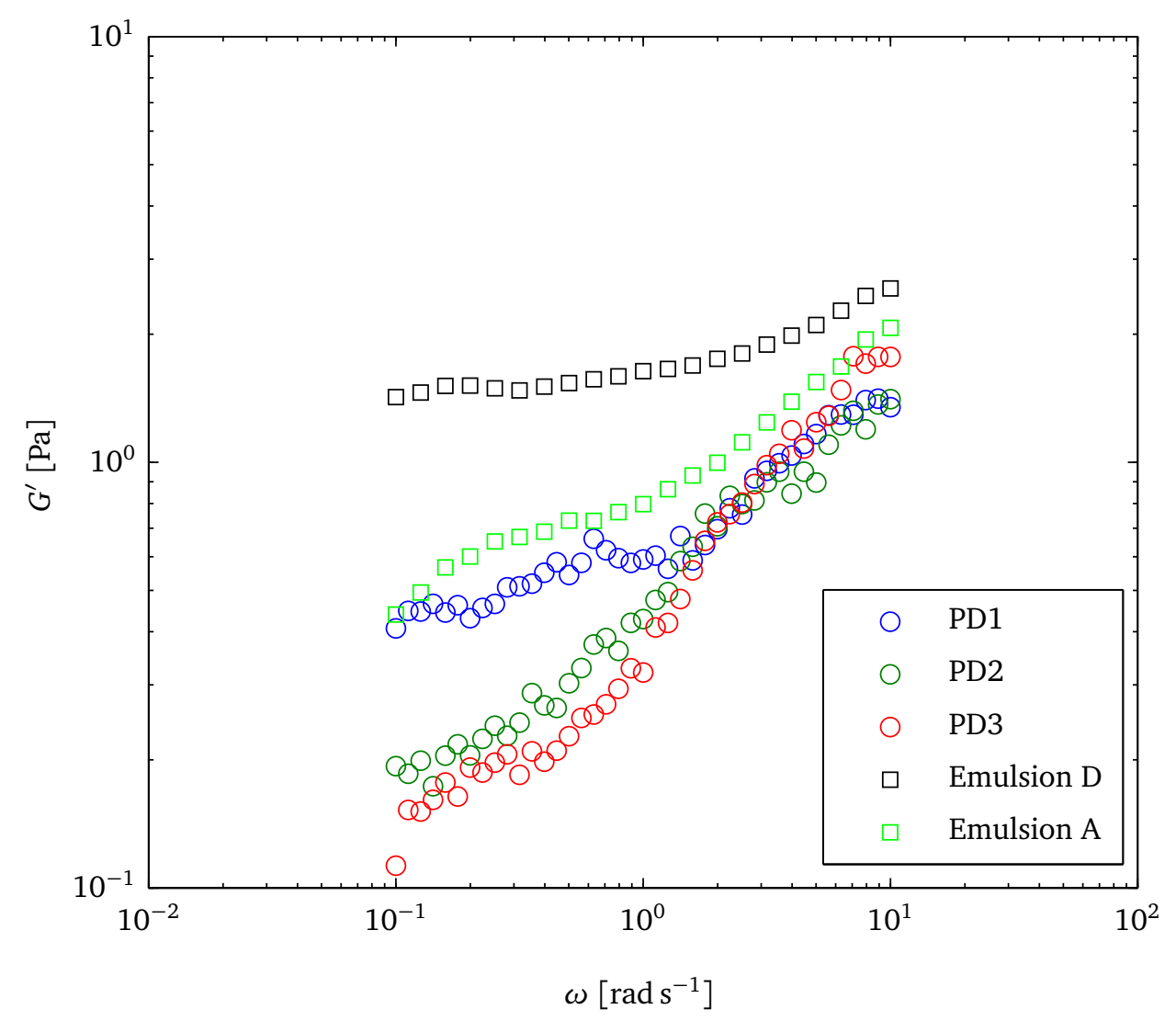

Figure 6.14: Frequency sweep storage moduli for PD1, PD2 and PD3 compared to emulsions A and D for $\gamma=1 \times 10^{-3}$.

larger droplets as being more deformable than smaller ones, but at the length scales and volume fractions probed here, it does not control the deformation. Indeed, at higher shear rates the emulsions converge to a similar stress response.

Instead of a plateau, emulsion A shows a sharp minimum centered at approximately $6 \times 10^{-3} \mathrm{~s}^{-1}$. Several experiments were performed to confirm this as a genuine feature of the flow curve and not an experimental anomaly. This behaviour suggests a sudden deformation of the droplet or rearrangement of the structure that could be caused by the droplets being compressed into a non-equilibrium shape by the measuring geometry. By observing a sample of emulsion A held between two glass slides separated by $400 \mu \mathrm{m}$, we were able to establish that it is unlikely the droplets were non-spherical. However, without performing rheo optical studies, the presence of a non-equilibrium structure at very low shear rates cannot be ruled out.

The tendency for emulsions with larger droplets to show hysteresis in their flow curves can be rationalised by considering the relaxation time for a deformed droplet, which is given by $[115,158]$

$$
\tau_{\text {relax }}=\frac{\eta_{c} a}{\xi}\left(\frac{(19 p+16)(2 p+3)}{40(p+1)}\right),
$$

which gives $\tau \approx 4$ to $11 \mathrm{~s}$ for the emulsions studied here. If this were the case, then no thixotropy would be observed. However, in soft glassy materials the relaxation 


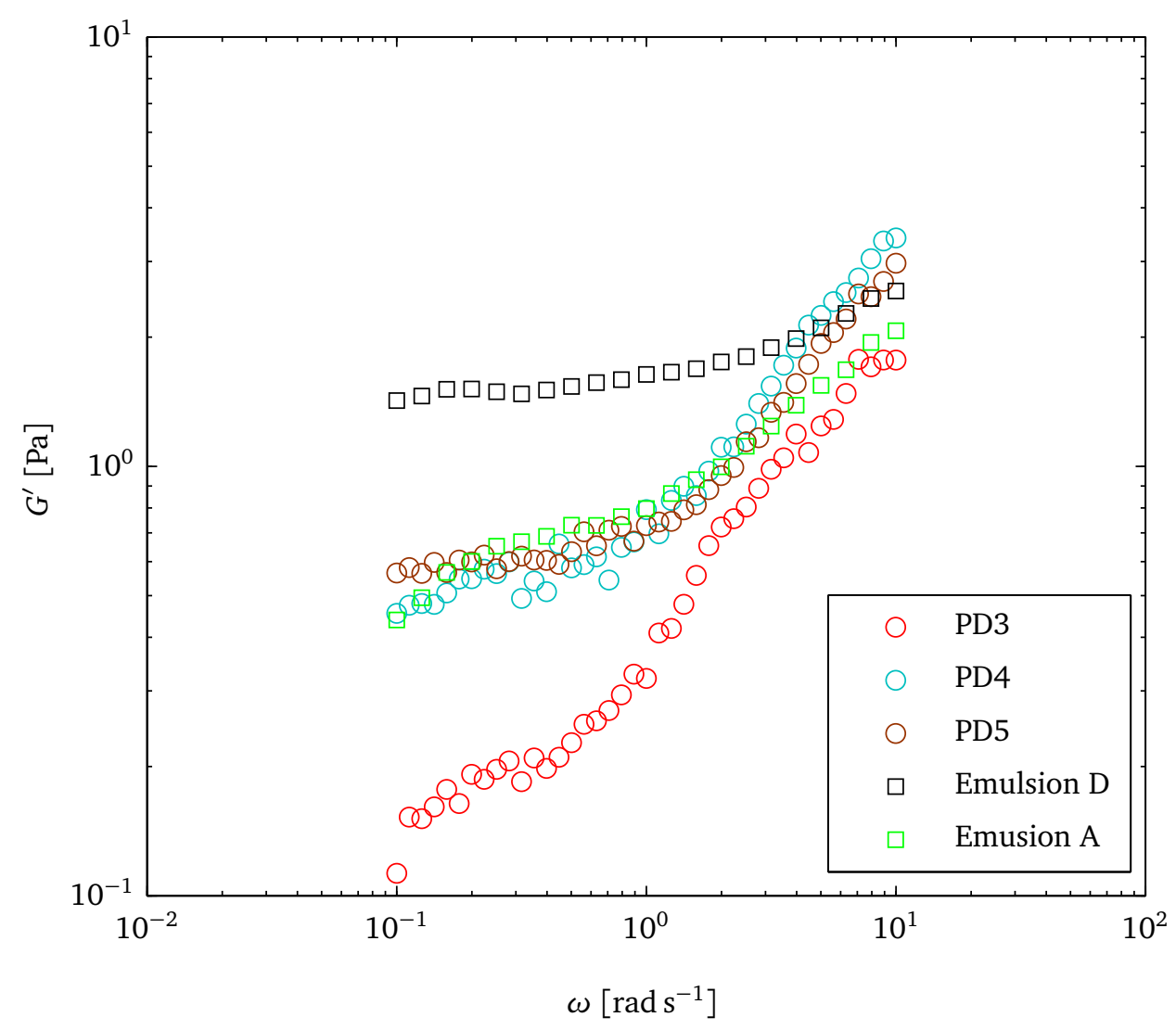

Figure 6.15: Frequency sweep storage moduli for PD3, PD4 and PD5 compared to emulsions A and D for $\gamma=1 \times 10^{-3}$.

of the sample to its equilibrium structure is a combination of two effects [159]: the interfacial tension relaxation mentioned above, which increases as the structure becomes more dense [160], and rearrangements of the droplets. Previous studies on monodisperse emulsion with sub-micrometre droplet radii showed no thixotropy $[40,125]$. Conversely, foams that typically have bubble radii in the order of several hundred micrometres show large degrees of thixotropy [161-163].

The transition from one mode of behaviour to another is also evident in the oscillatory strain sweep data, where the storage modulus for emulsions A-C is nearly identical within the LVE range, and only increases for the smaller droplets in emulsions $\mathrm{D}$ and $\mathrm{E}$, which themselves produce similar responses. While this trend is not repeated for the loss modulus, the frequency sweep experiments reinforce the idea that emulsions A-C are less elastic than D and E. Within these two domains there is no specific scaling of the storage modulus with the Laplace pressure of the droplets. As with the steady shear data, this could be a resolution issue, where the differences between, for example, emulsions $\mathrm{A}$ and $\mathrm{B}$ are within the experimental uncertainty associated with those systems.

Despite the lack of quantitative conclusions, previous observations combined with our results suggest that there is a critical ratio of the Laplace pressure to the interfacial tension for a given system supporting the unifying theory of soft glassy matter. Aside from influencing the extent of the initial deformation, the Laplace pressure drives a droplet to maximum expansion, while the interfacial tension serves to contract it. 


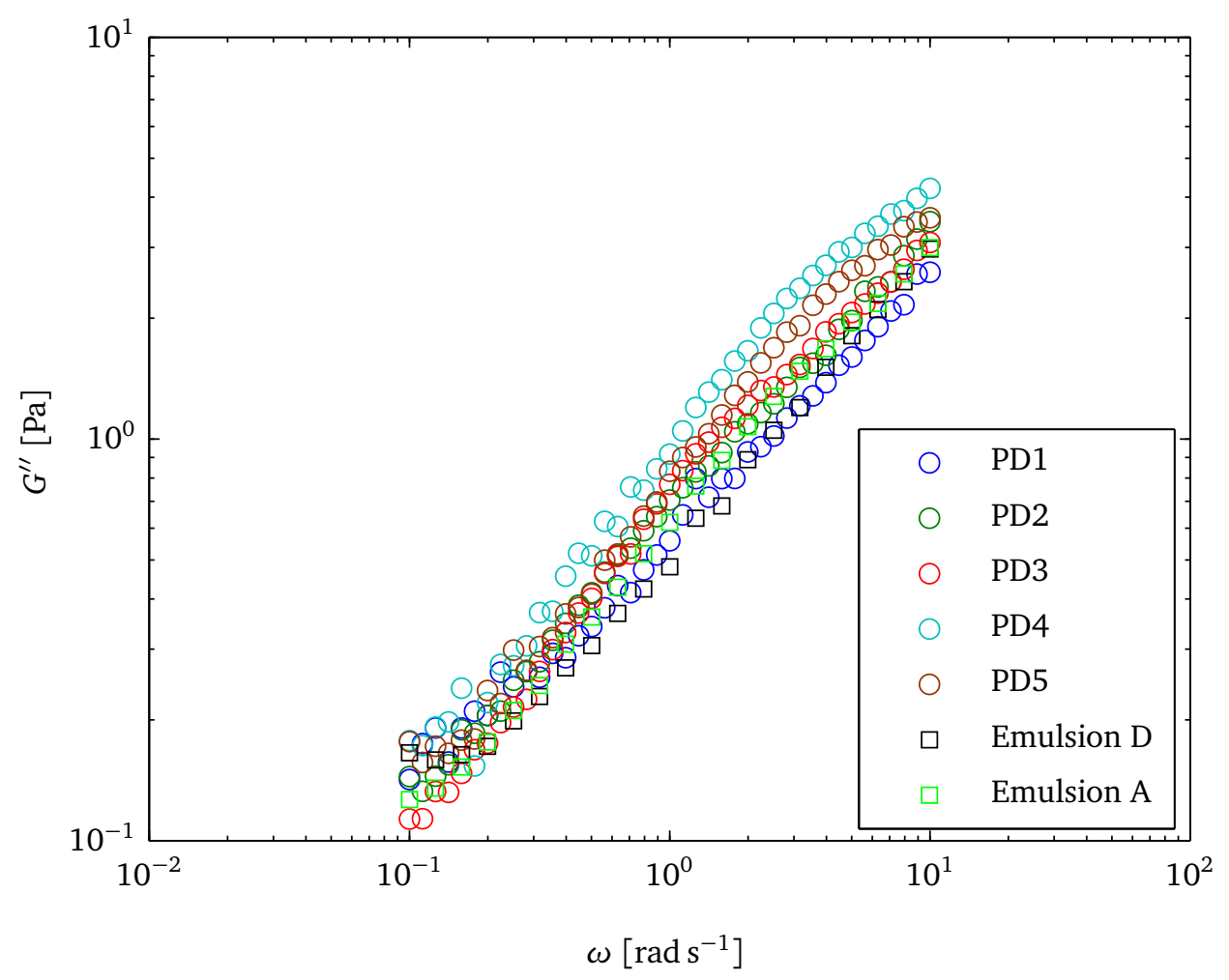

Figure 6.16: Frequency sweep loss moduli for all bimodal emulsions.

Below the critical value, the interfacial tension dominates the relaxation properties and contracts the droplet quickly after the cessation of deformation, much like in a conventional emulsion. Conversely, if the Laplace pressure is more significant, the droplet will not contract as quickly and there will be thixotropic behaviour. By tuning only the droplet size we have observed a transition from behaviour typically associated with emulsions to foam-like rheology.

\subsubsection{Bimodal emulsions}

While the steady shear data is inconclusive, oscillatory experiments suggest that polydispersity creates states in emulsion systems that cannot be considered perturbations or summations of constituent monodisperse emulsions. In general, PD1 with the smallest number fraction of emulsion D can be considered a perturbation of emulsion A. This is most likely because the presence of a low number of smaller droplets does not have a significant effect on the packing of the emulsion, and therefore the ability of the droplets to rearrange when sheared.

Emulsions PD2 and PD3 with $x_{D}=0.25$ and 0.5, respectively, represent an essentially new system created by the polydispersity. In all oscillatory tests, their storage moduli are lower than all other bimodal and monodisperse emulsions, sometimes by up to an order of magnitude. The exact ratio of droplets that induces this change of state is unknown. Diffusive diffraction of the continuous phase in both emulsions gave $q$-space plots that showed coherence features associated with the hexagonal close packing of droplets from emulsion A. This suggests the microstructure is comprised of domains of emulsion A that are perturbed by the smaller droplets of emulsion D in such a way that the ability of the sample to store energy elastically is significantly 
reduced. The monodisperse emulsions already lack significant resistance to flow, and this perturbation further lowers that barrier.

Counterintuitively, when $x_{D} \geq 0.75$ the emulsions do not develop behaviour reminiscent of emulsion D. Instead, they behave in a manner that most resembles emulsion A. It is difficult to rationalise this behaviour based on a qualitative understanding of the structural order in the system. As discussed in chapter 5, diffusive diffraction of the continuous phase in PD5 showed that there were no peaks associated with hexagonal close packing of either constituent emulsion. Despite this, the storage modulus of PD5 is similar to that of PD4 and emulsion A at low strain amplitudes. This suggests that the response of the larger droplets completely masks any contribution from those that are smaller. The apparent role of the smaller droplets in the rheological properties of these bimodal emulsions is limited to when they induce enough of a structural change that a new system is created.

Very little work has been performed on elucidating the effect of polydispersity on emulsions and colloidal systems as a whole. What work has been done has focused on the effect of widening the size distribution of an already polydisperse system. According to Chang and Powell [164], increasing the width of the size distribution should result in a decrease in the stress response and $G^{\prime}$ due to the greater ease in which particles in a suspension can occupy interstitial spaces within the structure and the lowered barriers to movement. This was verified by Luckham and Ukeje [165] using polystyrene beads.

While the difference in size between the two droplets is not large enough for the smaller droplets to occupy interstitial space, it is possible to rationalise the differences in the flow properties of the three "new" systems created by the polydispersity using concepts developed to explain the elastic behaviour of crystalline solids. Figure 5.15 shows that grain boundaries form between different regions of hexagonally packed droplets and that the presence of perturbative droplets increases the likelihood that grain boundaries will meet at a point. This significantly reduces the elastic strength of the structure, because grain boundaries act as a weak point for strain to propagate through the sample. If they meet at a point, then the strain is likely to propagate through more of the structure and in different directions [166].

By perturbing a matrix of large droplets with a modest number of smaller droplets as in PD1, insufficient points of weakness are created in the sample to significantly affect its flow properties. However, once the number fraction approaches 1:1, a large number of weak points have been created, and strain is able to propagate through all three dimensions of the sample with less resistance than it would have in an unperturbed system.

Emulsions PD4 and PD5 can be considered matrices of small droplets perturbed by larger ones, and PGSE NMR data in chapter 5 indicate that such a configuration experiences greater structural disorder than a matrix of larger droplets perturbed by smaller ones. The initial expectation would be that these emulsions would have the least resistance to flow. However, using a concept used in the work hardening of alloys [167] it is possible to understand why these emulsions have similar flow behaviour to monodisperse samples. Once a critical number of topological defects has been created, it is actually impossible for the grain boundaries to propagate and the elasticity of the sample increases. Obviously the number of large droplets required to produce this 
behaviour in a matrix of smaller droplets is less than for the reversed situation.

\subsection{Conclusion}

Rheological measurements using steady and oscillatory shear have shown that droplet size can have unexpected effects on the flow properties of an emulsion. By increasing the droplet size it is possible to move from an emulsion that is primarily elastic $\left(\delta=17^{\circ}\right)$ and does not display thixotropy under steady shear to a foam-like system that is near the gel point $\left(\delta=40^{\circ}\right)$ and displays a large degree of thixotropy. This is achieved through a shift in the forces responsible for the droplet deformation and relaxation, as well as the confined nature of the structure. These results support the concept of a soft glassy material whose properties are primarily controlled by the disordered nature of the system, rather than its exact chemical composition.

The rheological characterisation of bimodal emulsions suggests that it is not possible to consider the effect of polydispersity as a simple perturbation of a base system or the proportional combination of several monodisperse systems. Instead, it has been shown that the large droplets dominate the rheological behaviour at low and high number fractions of emulsion D and the significantly different behaviour at mid to low number fractions is the result of the creation of a new system. Further experimental work is required to establish the points of transition of these transitions, and which physicochemical properties of the bimodal or polydisperse systems induce the observed behaviour. 
It is hard to fail, but it is worse never to

have tried to succeed.

Theodore "Teddy" Roosevelt

(1858-1919)

\section{Conclusions and prospects}

\subsection{Conclusions}

A flow focusing microfluidic device has been used to generate monodisperse emulsions with droplet radii ranging from 20 to $50 \mu \mathrm{m}$ as determined by a combination of optical microscopy and diffusion NMR experiments using both the dispersed and continuous phases. The size of the droplets was limited by the width of the orifice in the junction of the microfluidic device until increased shear stresses induced by high continuous phase flow rates caused the droplets to form via dripping and jetting mechanisms. To our knowledge, this is the first time all three droplet formation modes have been observed using oil-in-water systems. By mixing two of these monodisperse emulsions over a range of droplet number fractions, bimodal emulsions that represent a first approximation to true polydispersity were created. Diffusion of the continuous phase in the polydisperse emulsions showed that a greater amount of structural disorder was induced when large droplets perturbed a matrix of smaller ones compared to the reversed situation.

Rheological characterisation of the monodisperse emulsions showed that it is possible to change between foam- and emulsion-like behaviour simply by altering the droplet size. In steady shear experiments, emulsions with radii from 50 to $37 \mu \mathrm{m}$ showed yielding and thixotropic behaviour typical of concentrated foams, while emulsions with radii of 33 and $30 \mu \mathrm{m}$ showed no thixotropic effects, which is typical of emulsion systems. This division was emphasised by oscillatory measurements, where emulsions with radii from 37 to $50 \mu \mathrm{m}$ had almost identical elastic character and were close to the gel point at low strains. Only once the droplet size decreased did the emulsions become predominantly elastic at low strain amplitudes. Unfortunately the resolution of the data was not sufficient to characterise the emulsions individually within these groups.

Bimodal emulsions were also characterised using steady and oscillatory shear to determine how polydispersity affects the flow properties of emulsions. The five samples created by mixing two monodisperse emulsions with radii of 50 and $33 \mu \mathrm{m}$ together exhibited three separate responses. The first comprised of PD1, which had a number fraction of 0.9:0.1 large to small droplets. Continuous phase diffusion NMR suggested that this emulsion had an ordered structure, and it behaved similarly to emulsion A. Further increase of the number fraction of emulsion $D$ to $0.75: 0.25$ and 1:1 created an entirely new system with a significant amount of disorder. This structural disorder served to decreased the elasticity of the sample significantly. Emulsions PD4 and PD5, where the large to small droplet ratio was 0.25:0.75 and 0.1:0.9, respectively, showed 
behaviour under steady and oscillatory shear comparable to that of emulsion A, the monodisperse emulsion with the largest droplets, despite having a majority of small droplets and a high degree of structural disorder. This seemingly anomalous behaviour was explained using the concepts of defect propagation within crystalline solids. This shows that polydispersity has a significant impact on emulsion rheology, and in a way that may not seem obvious or intuitive.

\subsection{Future prospects}

The chance to extend the time allocated to this research would enable many of the limitations of the current work to be rectified. Perhaps the largest limitation is the range of droplet sizes available with a microfluidic device of this type. By using a non-surface modified flow focusing device with a smaller orifice, it would be possible to produce emulsions having droplet sizes more typical of those encountered in the real world. This would have a secondary benefit. An oil with a high viscosity could be selected to not only further expand the range of sizes available, but to increase the accuracy of the measurements at low shear rates, strain amplitudes and oscillatory frequencies.

This would also enable the use of additional rheological experiments to probe the influence of polydispersity. Creep and stress relaxation experiments did not give physically sensible data for the emulsions studied in this research programme. Such tests could be used to determine how polydispersity affects the ability of the emulsion to structurally rearrange and the timescale(s) at which this occurs. The use of smaller droplets would also mean that rheo NMR could be used to characterise the influence of polydispersity on the spatial velocity of the droplets when subjected to shear.

If small enough droplets could be created, then it would be possible to explore the effect of polydispersity as a function of dispersed phase volume fractions. This was not possible in the current research, as the droplets creamed within seconds of emulsification. Additionally, it would be interesting to determine how the effect of polydispersity scales with the droplet size ratio. If necessary, techniques such as fractionation or shearing of coarse emulsions may need to be used to obtain droplets of a small enough size. While the eventual goal is to understand "true" polydisperse systems, it would be sensible to understand the complexities of bimodal systems first. 


\section{References}

[1] J. Bibette, F. Leal-Calderon, and P. Poulin, "Emulsions: Basic principles," Rep. Prog. Phys. 62 (1999) 969 - 1034.

[2] P. Becher, Emulsions: Theory and Practice. Oxford University Press, 2001.

[3] L. L. Schramm and E. N. Stasiuk, "Emulsions: Overview," in Finely dispersed particles, A. M. Spasic and J. P. Hsu, eds. CRC Press, 2006.

[4] D. J. McClements, Food Emulsions: Principles, Practice, and Techniques. CRC Press, 2005.

[5] R. J. Whitehurst, Emulsifiers in Food Technology. Blackwell Publishing Limited, 2004.

[6] P. Walstra, Physical Chemistry of Foods. Marcel Decker, 2003.

[7] H. A. Barnes, "Rheology of emulsions - a review," Colloids and Surfaces A 91 (1994) $89-95$.

[8] T. G. Mason, "New fundamental concepts in emulsion rheology," Current Opinion in Colloid \& Interface Science 4 (1999) 231 - 238.

[9] R. Pal, "Effect of droplet size on the rheology of emulsions," AIChE Journal 42 (1996) 3181-3190.

[10] T. G. Mason, A. H. Krall, H. Gang, J. Bibette, and D. A. Weitz, "Monodisperse emulsions: Properties and uses," in Encyclopedia of Emulsion Technology, P. Becher, ed. CRC Press, 1996.

[11] C. Zhou, P. Yue, and J. J. Feng, "Formation of simple and compound drops in microfluidic devices," Physics of Fluids 18 (2006) 92105-1 - 92105-14.

[12] T. F. Tadros, "Fundamental principles of emulsion rheology and their applications," Colloids and Surfaces A 91 (1994) 39 - 55.

[13] J. C. McDonald and G. M. Whitesides, "Poly(dimethylsiloxane) as a material for fabricating microfluidic devices," Accounts of Chemical Research 35 (2002) 491 499.

[14] E. M. Purcell, "Life at low reynolds number," American Journal of Physics 45 (1977) $3-11$.

[15] G. K. Batchelor, An introduction to fluid dynamics. Cam, 2000.

[16] H. Bruus, Theoretical Microfluidics. Oxford University Press, 2008.

[17] G. F. Christopher and S. L. Anna, "Microfluidic methods for generating continuous droplet streams," Journal of Physics D: Applied Physics 40 (2007) R319 - R336.

[18] M. Ulmeanu, "Preparation and characterization of water in oil emulsion via drop break-off," Colloids and Surfaces A 316 (2008) 119 - 124. 
[19] T. M. Squires, "Microfluidics: Fluid physics at the nanoliter scale," Rev. Mod. Phys. 77 (2005) $977-1026$.

[20] S. Y. Teh, R. Lin, L. H. Hung, and A. P. Lee, "Droplet microfluidics," Lab on a Chip 8 (2008) $198-220$.

[21] H. Becker and L. E. Locascio, "Polymer microfluidic devices," Talanta 56 (2002) 267-287.

[22] P. B. Umbanhowar, V. Prasad, and D. A. Weitz, "Monodisperse emulsion generation via drop break off in a coflowing stream," Langmuir 16 (2000) 347 351.

[23] C. Cramer, P. Fischer, and E. J. Windhab, "Drop formation in a co-flowing ambient fluid," Chemical Engineering Science 59 (2004) 3045 - 3058.

[24] Y. Hong and F. Wang, "Flow rate effect on droplet control in a co-flowing microfluidic device," Microfluidics and Nanofluidics 3 (2007) 341-346.

[25] S. L. Anna, N. Bontoux, and H. A. Stone, "Formation of dispersions using "flow focusing" in microchannels," Applied Physics Letters 82 (2003) 364 - 366.

[26] P. Garstecki, M. J. Fuerstman, H. A. Stonec, and G. M. Whitesides, "Formation of droplets and bubbles in a microfluidic t-junction-scaling and mechanism of break-up," Lab on a Chip 6 (2006) 437 - 446.

[27] J. H. Xu, S. W. Li, J. Tan, Y. J. Wang, and G. S. Luo, "Preparation of highly monodisperse droplet in a t-junction microfluidic device," AIChE Journal 52 (2006) 3005 - 3010.

[28] M. de Menech, P. Garstecki, F. Jousse, and H. A. Stone, "Transition from squeezing to dripping in a microfluidic t-shaped junction," Journal of Fluid Mechanics 595 (2008) 141 - 161.

[29] J. Husny and J. J. Cooper-White, "The effect of elasticity on drop creation in t-shaped microchannels," Journal of Non-Newtonian Fluid Mechanics 137 (2006) $121-136$.

[30] G.-B. Lee, C.-C. Chang, S.-B. Huang, and R.-J. Yang, "The hydrodynamic focusing effect inside rectangular microchannels," Journal of Micromechanics and Microengineering 16 (2006) 1024.

[31] S. L. Anna and H. C. Mayer, "Microscale tipstreaming in a microfluidic flow focusing device," Physics of Fluids 18 (2006) no. 12, 121512.

[32] A. S. Utada, E. Lorenceau, D. R. Link, P. D. Kaplan, H. A. Stone, and D. A. Weitz, "Monodisperse Double Emulsions Generated from a Microcapillary Device," Science 308 (2005) 537 - 541.

[33] Q. Xu and M. Nakajima, "The generation of highly monodisperse droplets through the breakup of hydrodynamically focused microthread in a microfluidic device," Applied Physics Letters 85 (2004) 3726 - 3728. 
[34] P. Garstecki, H. A. Stone, and G. M. Whitesides, "Mechanism for flow-rate controlled breakup in confined geometries: A route to monodisperse emulsions," Physical Review Letters 94 (2005) 164501.

[35] R. W. Hartel, Physical Chemistry of Foods. Marcel Dekker, 1992.

[36] R. A. D. Bruijn, "Tipstreaming of drops in simple shear flows," Chemical Engineering Science 48 (1993) 277 - 284.

[37] J. Bibette, D. Roux, and F. Nallet, "Depletion interactions and fluid-solid equilibrium in emulsions," Physical Review Letters 65 (1990) 2470 - 2473.

[38] M. P. Aronson, "The role of free surfactant in destabilizing oil-in-water emulsions," Langmuir 5 (1989) 494 - 501.

[39] J. Bibette, "Depletion interactions and fractionated crystallization for polydisperse emulsion purification," Journal of Colloid and Interface Science 147 (1991) $474-478$.

[40] T. G. Mason and J. Bibette, "Emulsification in viscoelastic media," Physical Review Letters 77 (1996) 3481-3484.

[41] C. Mabille, F. Leal-Calderon, J. Bibette, and V. Schmitt, "Monodisperse fragmentation in emulsions: Mechanisms and kinetics," Europhysics Letters 61 (2003) $708-714$.

[42] T. G. Mason and J. Bibette, "Shear rupturing of droplets in complex fluids," Langmuir 13 (1997) 4600 - 4613.

[43] C. Mabille, V. Schmitt, P. Gorria, F. Leal Calderon, V. Faye, B. Deminiere, and J. Bibette, "Rheological and shearing conditions for the preparation of monodisperse emulsions," Langmuir 16 (2000) 422 - 429.

[44] M. H. Levitt, Spin dynamics: basics of nuclear magnetic resonance. John Wiley \& Sons, 2 ed., 2008.

[45] R. R. Ernst, G. Bodenhausen, and A. Wokaun, Principles of Nuclear Magnetic Resonance in One and Two Dimensions. Ox, 1990.

[46] R. Pecora, "Basic Concepts - Scattering and Time Correlation Functions," in Soft Matter Characterization, R. Borsali and R. Pecora, eds. Springer Netherlands, 2008.

[47] P. T. Callaghan, Principles of Nuclear Magnetic Resonance Microscopy. Oxford University Press, 1991.

[48] R. Lenk, Fluctuations, Diffusion, and Spin Relaxation. Elsevier, 1986.

[49] A. Einstein, "Uber die von der molekularkinetischen theorie der warme geforderte bewegung von in ruhenden flüssigkeiten suspendierten teilchen," Annalen der Physik 322 (1905) 549Ü-560.

[50] H. Y. Carr and E. M. Purcell, "Effects of diffusion on free precession in nuclear magnetic resonance experiments," Physical Review 94 (1954) 630-638. 
[51] R. J. Nelson, Y. Maguire, D. F. Caputo, G. Leu, Y. Kang, M. Pravia, D. Tuch, Y. S. Weinstein, and D. G. Cory, "Counting echoes: application of a complete reciprocal-space description of NMR spin dynamics," Concepts in Magnetic Resonance A 10 (1998) 331-341.

[52] E. L. Hahn, “Spin echoes," Physical Review 80 (1950) 580-594.

[53] E. O. Stejskal and J. E. Tanner, "Spin diffusion measurements: Spin echoes in the presence of a time-dependent field gradient," Journal of Chemical Physics 42 (1965) $288-292$.

[54] J. E. Tanner, "Use of the Stimulated Echo in NMR Diffusion Studies," Journal of Chemical Physics 52 (1970) 2523-2526.

[55] J. E. Tanner, "Erratum: Use of the Stimulated Echo in NMR Diffusion Studies," The Journal of Chemical Physics 57 (1972) 3586-3586.

[56] W. S. Price, NMR Studies of Translational Motion: Principles and Applications. Cambridge University Press, 2009.

[57] H. C. Torrey, "Bloch equations with diffusion terms," Physical Revi 104 (1956) $563-565$.

[58] D. C. Douglass and D. W. McCall, "Diffusion in paraffin hydrocarbons," Journal of Physical Chemistry 62 (1958) 1102-1107.

[59] J. E. Tanner and E. O. Stejskal, "Restricted Self-Diffusion of Protons in Colloidal Systems by the Pulsed-Gradient, Spin-Echo Method," The Journal of Chemical Physics 49 (1968) 1768-1777.

[60] N. N. Yadav and W. S. Price, "Impediments to the accurate structural characterisation of a highly concentrated emulsion studied using NMR diffusion diffraction," Journal of Colloid and Interface Science 338 (2009) 163-168.

[61] P. T. Callaghan, C. D. Eccles, and Y. Xia, "Nmr microscopy of dynamic displacements: k-space and q-space imaging," Journal of Physics E: Scientific Instruments 21 (1988) 820-822.

[62] W. S. Price, "Pulsed-field gradient nuclear magnetic resonance as a tool for studying translational diffusion: Part 1. Basic theory," Concepts in Magnetic Resonance A 9 (1997) 299 - 336.

[63] J. S. Murday and R. M. Cotts, "Self-diffusion coefficient of liquid lithium," The Journal of Chemical Physics 48 (1968) 4938-4945.

[64] P. T. Callaghan, S. L. Codd, and J. D. Seymour, "Spatial coherence phenomena arising from translational spin motion in gradient spin echo experiments," Concepts in Magnetic Resonance 11 (1999) 181-202.

[65] P. T. Callaghan, D. MacGowan, K. J. Packer, and F. O. Zelaya, "High-resolution q-space imaging in porous structures," Journal of Magnetic Resonance 90 (1990) 177-182. 
[66] P. T. Callaghan, A. Coy, D. MacGowan, K. J. Packer, and F. O. Zelaya, "Diffraction-like effects in nmr diffusion studies of fluids in porous solids," Nature 351 (1991) 467-469.

[67] B. Balinov, B. Jonsson, P. Linse, and O. Soderman, "The NMR Self-Diffusion Method Applied to Restricted Diffusion. Simulation of Echo Attenuation from Molecules in Spheres and between Planes," Journal of Magnetic Resonance, Series A 104 (1993) 17-25.

[68] A. Coy and P. T. Callaghan, "Pulsed gradient spin echo nuclear magnetic resonance for molecules diffusing between partially reflecting rectangular barriers," The Journal of Chemical Physics 101 (1994) 4599-4609.

[69] M. Johns, "NMR studies of emulsions," Current Opinion in Colloid \& Interface Science 14 (2009) 178-183.

[70] M. L. Johns and K. G. Hollingsworth, "Characterisation of emulsion systems using NMR and MRI," Progress in Nuclear Magnetic Resonance Spectroscopy 50 (2007) $51-70$.

[71] K. J. Packer and C. Rees, "Pulsed NMR studies of restricted diffusion. I. Droplet size distributions in emulsions," Journal of Colloid and Interface Science 40 (1972) $206-218$.

[72] P. T. Callaghan, K. W. Jolley, and R. S. Humphrey, "Diffusion of fat and water in cheese as studied by pulsed field gradient nuclear magnetic resonance," Journal of Colloid and Interface Science 93 (1983) 521 - 529.

[73] L. Ambrosone, A. Ceglie, G. Colafemmina, and G. Palazzo, "A novel approach for determining the droplet size distribution in emulsion systems by generating function," Journal of Chemical Physics 107 (1997) 10756 - 10763.

[74] L. Ambrosone, A. Ceglie, G. Colafemmina, and G. Palazzo, "General methods for determining the droplet size distribution in emulsion systems," Journal of Chemical Physics 110 (1999) 797 - 804.

[75] K. G. Hollingsworth and M. L. Johns, "Measurement of emulsion droplet sizes using PFG NMR and regularization methods," Journal of Colloid and Interface Science 258 (2003) 383 - 389.

[76] A. Woodward, T. Cosgrove, J. Espidel, P. Jenkinsb, and N. Shawb, "Monodisperse emulsions from a microfluidic device, characterised by diffusion NMR," Soft Matter 3 (2007) 627 - 633.

[77] I. Fourel, J. P. Guillement, and D. L. Botlan, "Determination of Water Droplet Size Distributions by Low Resolution PFG-NMR: I. Liquid Emulsions," Journal of Colloid and Interface Science 164 (1994) 48-53.

[78] M. H. Chestnut, "Confocal microscopy of colloids," Current Opinion in Colloid and Interface Science 2 (1997) 158-161. 
[79] D. J. McClements and J. N. Coupland, "Theory of droplet size distribution measurements in emulsions using ultrasonic spectroscopy," Colloids and Surfaces A: Physicochemical and Engineering Aspects 117 (1996) 161-170.

[80] R. G. Larson, The Structure and Rheology of Complex Fluids. Oxford University Press, 1998.

[81] C. Macosko, Rheology: Principles, Measurements, and Applications. Wiley-VCH, 1994.

[82] F. Morrison, Understanding Rheology. Oxford University Press, 2001.

[83] M. Yamamoto, "The Visco-elastic Properties of Network Structure III. Normal Stress Effect (Weissenberg Effect)," Journal of the Physical Society of Japan 13 (1958) 1200-1211.

[84] G. S. Beavers and D. D. Joseph, "Novel weissenberg effects," Journal of Fluid Mechanics Digital Archive 81 (1977) 265-272.

[85] R. Chhabra, Bubbles, Drops, and Particles in Non-Newtonian Fluids. CRC Press, 2 ed., 2006.

[86] M. Reiner, Deformation, Strain and Flow. Wiley, 1960.

[87] R. B. Bird, "Useful Non-Newtonian Models," Annual Review of Fluid Mechanics 8 (1976) 13-34.

[88] W. W. Graessley, "Viscosity of entangling polydisperse polymers," The Journal of Chemical Physics 47 (1967) no. 6, 1942-1953.

[89] R. H. Colby, D. C. Boris, W. E. Krause, and S. Dou, "Shear thinning of unentangled flexible polymer liquids," Rheologica Acta 46 (2007) 569-575.

[90] A. C. Rust and M. Manga, "Effects of bubble deformation on the viscosity of dilute suspensions," Journal of Non-Newtonian Fluid Mechanics 104 (2002) 53-63.

[91] D. J. Stein and F. J. Spera, "Rheology and microstructure of magmatic emulsions: theory and experiments," Journal of Volcanology and Geothermal Research 49 (1992) 157-174.

[92] M. Ballard, R. Buscall, and F. Waite, "The theory of shear-thickening polymer solutions," Polymer 29 (1988) 1287-1293.

[93] H. A. Barnes, "Shear-Thickening ("Dilatancy") in Suspensions of Nonaggregating Solid Particles Dispersed in Newtonian Liquids," Journal of Rheology 33 (1989) 329-366.

[94] W. Ostwald, "Ueber die Geschwindigkeitsfunktion der Viskosität disperser Systeme. I," Kolloid Zeitschrift 36 (1925) 99-117.

[95] W. Herschel and R. Bulkley, "Measurement of consistency as applied to rubber-benzene solutions," Proceedings of the American Society for the Testing of Materials 26 (1926) 621-633. 
[96] P. C. F. Moller, J. Mewis, and D. Bonn, "Yield stress and thixotropy: on the difficulty of measuring yield stresses in practice," Soft Matter 2 (2006) 274-283.

[97] H. A. Barnes and K. Walters, "The yield stress myth?," Rheologica Acta 24 (1985) 323-326.

[98] R. Bird, R. Armstrong, and O. Hassager, Dynamics of polymeric liquids, vol. 1. Wiley, 2 ed., 1987.

[99] A. Papo, "Rheological models for cement pastes," Materials and Structures 21 (1988) 41-46.

[100] M. Reiner, “The deborah number," Physics Today 17 (1964) 62-62.

[101] J. C. Maxwell, "On the Dynamical Theory of Gases," Philosophical Transactions of the Royal Society 157 (1867) 49-88.

[102] A. Love, The Mathematical Theory of Elasticity Herschel1926. Cambridge University Press, 1927.

[103] R. M. Christensen, Theory of Viscoelasticity. Dover, 2003.

[104] T. G. Mezger, The Rheology Handbook. Vincentz, 2006.

[105] K. Bekkour, M. Leyama, A. Benchabane, and O. Scrivener, "Time-dependent rheological behavior of bentonite suspensions: An experimental study," Journal of Rheology 49 (2005) 1329-1345.

[106] W. Yu, M. Bousmina, M. Grmela, J.-F. Palierne, and C. Zhou, "Quantitative relationship between rheology and morphology in emulsions," Journal of Rheology 46 (2002) 1381-1399.

[107] R. Pal, "Viscous behavior of concentrated emulsions of two immiscible newtonian fluids with interfacial tension," Journal of Colloid and Interface Science 263 (2003) 296-305.

[108] P. Wilde, "Interfaces: their role in foam and emulsion behaviour," Current Opinion in Colloid \& Interface Science 5 (2000) 176-181.

[109] G. I. Taylor, "The Formation of Emulsions in Definable Fields of Flow," Proceedings of the Royal Society of London. Series A 146 (1934) 501-523.

[110] F. D. Rumscheidt and S. G. Mason, "Particle motions in sheared suspensions xii. deformation and burst of fluid drops in shear and hyperbolic flow," Journal of Colloid Science 16 (1961) 238-261.

[111] T. V. Vliet and J. Lyklema, "Rheology of polyelectrolyte-stabilized emulsions : I. Effects due to conformational properties of adsorbed polymethacrylate," Journal of Colloid and Interface Science 63 (1978) 97-105.

[112] T. van Vliet, J. Lyklema, and M. van den Tempel, "Rheology of polyelectrolyte-stabilized emulsions : II. Dynamic measurements," Journal of Colloid and Interface Science 65 (1978) 505-508. 
[113] G. I. Taylor, "The Viscosity of a Fluid Containing Small Drops of Another Fluid," Proceedings of the Royal Society of London. Series A 138 (1932) 41-48.

[114] M. A. Nawab and S. G. Mason, "Viscosity of dilute suspensions of thread-like particles," The Journal of Physical Chemistry 62 (1958) 1248-1253.

[115] J. G. Oldroyd, "The Elastic and Viscous Properties of Emulsions and Suspensions," Proceedings of the Royal Society of London. Series A 218 (1953) 122-132.

[116] S. R. Derkach, "Rheology of emulsions," Advances in Colloid and Interface Science 151 (2009) 1-23.

[117] N. A. Frankel and A. Acrivos, "The constitutive equation for a dilute emulsion," Journal of Fluid Mechanics Digital Archive 44 (1970) 65-78.

[118] S. J. Choi and W. R. Schowalter, "Rheological properties of nondilute suspensions of deformable particles," Physics of Fluids 18 (1975) no. -, 420-427.

[119] H. M. Princen, "The structure, mechanics, and rheology of concentrated emulsions and fluid foams," in Encyclopedic Handbook of Emulsion Technology, J. Sjb̈lom, ed. CRC Press, 2001.

[120] R. Pal, "Shear viscosity behavior of emulsions of two immiscible liquids," Journal of Colloid and Interface Science 225 (2000) 359-366.

[121] P. Coussot, H. Tabuteau, X. Chateau, L. Tocquer, and G. Ovarlez, "Aging and solid or liquid behavior in pastes," Journal of Rheology 50 (2006) 975-994.

[122] D. A. G. Bruggeman, "Berechnung verschiedener physikalischer konstanten von heterogenen substanzen. i. dielektrizitätskonstanten und leitfähigkeiten der mischkörper aus isotropen substanzen," Annalen der Physik 416 (1935) 636-664.

[123] R. Höhler, S. Cohen-Addad, and V. Labiausse, "Constitutive equation to describe the nonlinear elastic response of aqueous foams and concentrated emulsions," Journal of Rheology 48 (2004) no. 3, 679-690.

[124] T. G. Mason, J. Bibette, and D. A. Weitz, "Elasticity of compressed emulsions," Physical Review Letters 75 (1995) 2051 - 2054.

[125] T. G. Mason, J. Bibette, and D. A. Weitz, "Yielding and flow of monodisperse emulsions," Journal of Colloid and Interface Science 179 (1996) 439 - 448.

[126] R. Chanamai and D. J. McClements, "Dependence of creaming and rheology of monodisperse oil-in-water emulsions on droplet size and concentration," Colloids and Surfaces A: Physicochemical and Engineering Aspects 172 (2000) no. 1-3, 79-86.

[127] D. J. McClements and R. Chanamai, "Physicochemical properties of mono disperse oil-in-water emulsions," Journal of Dispersion Science and Technology 23 (2002) 125-134. 
[128] A. J. C. Ladd, "Hydrodynamic transport coefficients of random dispersions of hard spheres," The Journal of Chemical Physics 93 (1990) 3484-3494.

[129] D. R. Caudwell, J. P. M. Trusler, V. Vesovic, and W. A. Wakeham, "The Viscosity and Density of n-Dodecane and n-Octadecane at Pressures up to $200 \mathrm{MPa}$ and Temperatures up to 473 K," International Journal of Thermophysics 25 (2004) 1339-1352.

[130] W. Lee, L. M. Walker, and S. L. Anna, "Role of geometry and fluid properties in droplet and thread formation processes in planar flow focusing," Physics of Fluids 21 (2009) 032103.

[131] Y.-C. Tan and A. P. Lee, "Microfluidic separation of satellite droplets as the basis of a monodispersed micron and submicron emulsification system," Lab on a Chip 5 (2005) 1178-1183.

[132] Y.-C. Tan, Y. L. Ho, and A. P. Lee, "Microfluidic sorting of droplets by size," Microfluidics and Nanofluidics 4 (2008) 343-348.

[133] A. J. Woodward, Monodisperse emulsions from a microfluidic device: A diffusion NMR and SANS study. PhD thesis, University of Bristol, 2008.

[134] M. Holz, S. R. Heil, and A. Sacco, "Temperature-dependent self-diffusion coefficients of water and six selected molecular liquids for calibration in accurate 1H NMR PFG measurements," Physical Chemistry Chemical Physics 2 (2000) 4740-4742.

[135] P. T. Callaghan, A. Coy, T. P. J. Halpin, D. MacGowan, K. J. Packer, and F. O. Zelaya, "Diffusion in porous systems and the influence of pore morphology in pulsed gradient spin-echo nuclear magnetic resonance studies," The Journal of Chemical Physics 97 (1992) 651-662.

[136] A. I. Romoscanu, A. Fenollosa, S. Acquistapace, D. Gunes, T. Martins-Deuchande, P. Clausen, R. Mezzenga, M. Nydn, K. Zick, and E. Hughes, "Structure, Diffusion, and Permeability of Protein-Stabilized Monodispersed Oil in Water Emulsions and Their Gels: A Self-Diffusion NMR Study," Langmuir 26 (2010) 6184-6192.

[137] P. T. Callaghan, Translational Dynamics and Magnetic Resonance. Oxford University Press, 2010.

[138] B. Haakansson, R. Pons, and O. Söderman, "Diffraction-like effects in a highly concentrated w/o emulsion: a PFG NMR study," Magnetic Resonance Imaging 16 (1998) 643-646.

[139] P. W. Kuchel, T. R. Eykyn, and D. G. Regan, "Measurement of compartment size in q-space experiments: Fourier transform of the second derivative," Magnetic Resonance in Medicine 52 (2004) 907-912.

[140] R. K. McGeary, "Mechanical packing of spherical particles," Journal of the American Ceramic Society 44 (1961) 513-522. 
[141] Y. Shi and Y. Zhang, "Simulation of random packing of spherical particles with different size distributions," Applied Physics A: Materials Science \& Processing 92 (2008) 621-626.

[142] G. Ovarlez, S. Rodts, X. Chateau, and P. Cousso, "Phenomenology and physical origin of shear localization and shear banding in complex fluids," Rheologica Acta 48 (2009) 831-844.

[143] H. Egger and K. M. McGrath, "Estimating depletion layer thickness in colloidal systems: Correlation with oil-in-water emulsion composition," Colloids and Surfaces A: Physicochemical and Engineering Aspects 275 (2006) 107-113.

[144] R. Pal, "Slippage during the flow of emulsions in rheometers," Colloids and Surfaces A: Physicochemical and Engineering Aspects 162 (2000) 55-66.

[145] M. C. Sanchez, C. Valencia, J. M. Franco, and C. Gallegos, "Wall slip phenomena in oil-in-water emulsions: Effect of some structural parameters," Journal of Colloid and Interface Science 241 (2001) 226-232.

[146] J. M. Franco, C. Gallegos, and H. A. Barnes, "On slip effects in steady-state flow measurements of oil-in-water food emulsions," Journal of Food Engineering 36 (1998) 89-102.

[147] M. A. Riscardo, J. E. Moros, J. M. Franco, and C. Gallegos, "Rheological characterisation of salad-dressing-type emulsions stabilised by egg yolk/sucrose distearate blends," European Food Research and Technology 220 (2005) 380-388.

[148] P. Partal, A. Guerrero, M. Berjano, and C. Gallegos, "Transient flow of o/w sucrose palmitate emulsions," Journal of Food Engineering 41 (1999) 33-41.

[149] O. H. Campanella and M. Peleg, "Analysis of the transient flow of mayonnaise in a coaxial viscometer," Journal of Rheology 31 (1987) 439-452.

[150] P. Sollich, F. m. c. Lequeux, P. Hébraud, and M. E. Cates, "Rheology of soft glassy materials," Physical Review Letters 78 (1997) 2020-2023.

[151] P. Sollich, "Rheological constitutive equation for a model of soft glassy materials," Physical Review E 58 (1998) 738-759.

[152] M. Cloitre, R. Borrega, F. Monti, and L. Leibler, "Glassy dynamics and flow properties of soft colloidal pastes," Physical Review Letters 90 (2003) 068303.

[153] P. Hébraud and F. Lequeux, "Mode-coupling theory for the pasty rheology of soft glassy materials," Physical Review Letters 81 (1998) 2934-2937.

[154] H. Princen, "Rheology of foams and highly concentrated emulsions. ii. experimental study of the yield stress and wall effects for concentrated oil-in-water emulsions," Journal of Colloid and Interface Science 105 (1985) 150 $-171$. 
[155] G. Ovarlez, S. Rodts, A. Ragouilliaux, P. Coussot, J. Goyon, and A. Colin, "Wide-gap couette flows of dense emulsions: Local concentration measurements, and comparison between macroscopic and local constitutive law measurements through magnetic resonance imaging," Phys. Rev. E 78 (2008) 036307.

[156] J. Goyon, A. Colin, G. Ovarlez, A. Ajdari, and L. Bocquet, "Spatial cooperativity in soft glassy flows," Nature 454 (2008) 84-87.

[157] P. T. Callaghan, "Rheo NMR and shear banding," Rheologica Acta 47 (2008) 243-255.

[158] J. M. Rallison, "The Deformation of Small Viscous Drops and Bubbles in Shear Flows," Annual Review of Fluid Mechanics 16 (1984) 45-66.

[159] S. Cohen-Addad, R. Höhler, and Y. Khidas, "Origin of the slow linear viscoelastic response of aqueous foams," Physical Review Letters 93 (2004) 028302 .

[160] T. Mason, M. D. Lacasse, G. S. Grest, D. Levine, J. Bibette, and D. A. Weitz, "Osmotic pressure and viscoelastic shear moduli of concentrated emulsions," in Jamming and Rheology: Constrained Dynamics on Microscopic and Macroscopic Scales, A. J. Liu and S. R. Nagel, eds. CRC Press, 2001.

[161] Y. Jiang, P. J. Swart, A. Saxena, M. Asipauskas, and J. A. Glazier, "Hysteresis and avalanches in two-dimensional foam rheology simulations," Physical Review E: Statistical Physics, Plasmas, Fluids, and Related Interdisciplinary Topics 59 (1999) 5819-5832.

[162] K. B. O. Scrivener, "Time-dependent and flow properties of foams," Mechanics of Time-Dependent Materials 2 (1998) 171Ü-193.

[163] R. Hohler and S. Cohen-Addad, "Rheology of liquid foam," Journal of Physics: Condensed Matter 17 (2005) R1041.

[164] C. Chang and R. L. Powell, "Effect of particle size distributions on the rheology of concentrated bimodal suspensions," Journal of Rheology 38 (1994) 85-98.

[165] P. F. Luckham and M. A. Ukeje, "Effect of particle size distribution on the rheology of dispersed systems," Journal of Colloid and Interface Science 220 (1999) 347-356.

[166] P. Chakin and T. Lubensky, Principles of consdensed matter physics. Cam, 1995.

[167] J. W. Martin, Micromechanisms in Particle-Hardened Alloys. Cambridge University Press, 1980. 
